\title{
DYNAMIC ACCESS CLASS BARRING AND RELAY ASSISTED RADIO RESOURCE ALLOCATION METHODS FOR CELLULAR M2M NETWORKS
}

\author{
by \\ Lilatul Ferdouse \\ M.S., University of Dhaka, Bangladesh, 2007 \\ A thesis \\ presented to the Ryerson University \\ in partial fulfilment of the requirements for the degree of \\ Master of Applied Science in the \\ Program of Electrical and Computer Engineering \\ Toronto, Ontario, Canada, 2015 \\ (c) Lilatul Ferdouse, 2015
}




\section{AUTHORS DECLARATION FOR ELECTRONIC SUBMISSION OF A DISSERTATION}

I hereby declare that I am the sole author of this dissertation. This is a true copy of the dissertation, including any required final revisions, as accepted by my examiners.

I authorize Ryerson University to lend this dissertation to other institutions or individuals for the purpose of scholarly research.

I further authorize Ryerson University to reproduce this dissertation by photocopying or by other

means, in total or in part, at the request of other institutions or individuals for the purpose of scholarly research.

I understand that my dissertation may be made electronically available to the public. 


\title{
Abstract \\ Dynamic Access Class Barring and Relay Assisted Radio Resource Allocation Methods for Cellular M2M Networks
}

\author{
(C) Lilatul Ferdouse, 2015 \\ Master of Applied Science \\ Electrical and Computer Engineering \\ Ryerson University
}

Cellular based M2M systems generate massive number of access requests which create congestion in the cellular network. The contention-based random access procedures are designed for cellular networks which cannot accommodate a large number of M2M traffic. Moreover, M2M systems share same radio resources with cellular users. Resource allocation problem becomes a challenging issue in cellular M2M systems. In this thesis, we address these two problems by analyzing a contention-based slotted Aloha random access procedure for M2M networks using different performance metrics. The impact of massive M2M traffic over cellular traffic is studied based on different arrival rate, random access opportunity and throughput. An analytical model of selecting a base station (eNB) along with load balancing is developed. Finally, two methods have been presented and evaluated with M2M traffic. First one is dynamic access class barring method which controls RAN level congestion by selecting an appropriate eNB and applying load balancing method. Second one is relay-assisted radio resource allocation method which maximizes the sum throughput of the system by utilizing the available radio resource blocks and relay nodes to the MTC systems. Numerical results show that frame transmission rate influences the selection probability of the base stations. Moreover, the dynamic access class barring parameter along with frame transmission rate improve the overall throughput and access success probability among base stations as well as avoid overload situation in a particular base station. 


\section{Acknowledgment}

I would first like to thank my supervisor, Professor Alagan Anpalagn for his support, patience and time throughout my graduate studies. I am finally able to achieve my goal for his strong support and help. He did not only guide me in the course of my thesis, but also provided me an opportunity to be benefited from his vast knowledge. He was always present to help me out and to guide me whenever I needed his guidance.

I would like to acknowledge my gratitude to the Department of Electrical and Computer Engineering and the School of Graduate Studies of Ryerson University, for their financial supports and all the opportunities.

I would like to thank my parents for their support, love and advice. Without them, I would not be here today. Finally, and most importantly, I would like to thank my husband Mohammed Zahirul Hoq Sarker, who has been a constant source of encouragement for me throughout my studies. Without his support, courage and adorableness, I would not be able to achieve my goal. 


\section{Contents}

1 Introduction $\quad 1$

1.1 Context of our study . . . . . . . . . . . . . . . . . . . . . . 1

1.2 Research problem . . . . . . . . . . . . . . . . . . . 2

1.3 Objective and contributions of the thesis . . . . . . . . . . . . . 3

1.4 Thesis organization . . . . . . . . . . . . . . . . . . . . 3

2 Machine-Type Communication $\quad 5$

$2.1 \mathrm{M} 2 \mathrm{M}$ applications and service requirements . . . . . . . . . . . . . . . . . 6

2.2 M2M traffic characteristics . . . . . . . . . . . . . . . . . . . . 9

2.3 M2M architecture underlaying $3 \mathrm{GPP}$ networks . . . . . . . . . . . . . . 9

2.4 Challenging issues of M2M enable cellular networks . . . . . . . . . . . . 13

3 Survey of Massive Access Management Methods for MTC enabled Cellular $\begin{array}{ll}\text { Networks } & 16\end{array}$

3.1 Congestion in MTC over 3GPP Network . . . . . . . . . . . . . . . . . 16

3.1.1 Signalling congestion scenarios . . . . . . . . . . . . . . . 17

3.1.1.1 Overload situation in radio access network (RAN) . . . . . . 18

3.1.1.2 Core Network (CN) congestion scenario: . . . . . . . . . . . . . 19

3.1.2 Congestion control methods applied to RAN . . . . . . . . . . . . 19

3.1.3 Congestion control methods applied to $\mathrm{CN} \ldots \ldots$. . . . . . . . . 26

3.2 Comparison of different congestion and overload control techniques . . . . . . 32

4 Proposed Dynamic Access Class Barring Method 36 
4.1 Preliminary analysis of RA in M2M traffic . . . . . . . . . . . . 36

4.2 Analytical model . . . . . . . . . . . . . . . . . . . . . . . . . 39

4.2.1 System model and problem formulation . . . . . . . . . . . . . . . . 39

4.2 .2 Proposed solution . . . . . . . . . . . . . . . . . . . 40

4.2.2.1 Base station selection method . . . . . . . . . . . . 40

4.2.2.2 Load Balancing . . . . . . . . . . . . . . . . . . . 41

4.3 Proposed dynamic access class barring method . . . . . . . . . . . . . . 43

4.4 Simulation parameters $\ldots \ldots \ldots \ldots \ldots \ldots$

4.5 Simulation results . . . . . . . . . . . . . . . . . . . 45

4.5 .1 Impact of frame transmission rate . . . . . . . . . . . . . . 45

4.5 .2 Impact of dynamic ACB schema . . . . . . . . . . . . . . . . . 46

4.5.2.1 Group one: $F T R_{1}=F T R_{2} \ldots \ldots \ldots \ldots$

4.5.2.2 Group two: $F T R_{1}>F T R_{2} \ldots \ldots \ldots \ldots$

4.5.2.3 Group three: $F T R_{1}<F T R_{2} \ldots \ldots \ldots \ldots 2$

5 Relay Assisted Radio Resource Allocation Method for MTC Network $\quad 54$

5.1 Previous works $\ldots \ldots \ldots \ldots \ldots \ldots \ldots \ldots$

5.2 Radio resource block . . . . . . . . . . . . . . . . . . . 55

5.3 Relay assisted radio resource allocation method f . . . . . . . . . . 56

5.3 .1 System model . . . . . . . . . . . . . . . . . . . . . . 56

5.3 .2 Problem formulation . . . . . . . . . . . . . . 57

5.3 .3 Relay assisted radio resource allocation method . . . . . . . . . . . 59

5.3.4 Relay assisted radio resource allocation algorithm (RARRA) . . . . . . 60

5.4 Performance analysis of RARRA algorithm . . . . . . . . . . . . 63

5.4 .1 Impact of buffer size $(\aleph) \ldots \ldots \ldots \ldots 64 \ldots \ldots \ldots$

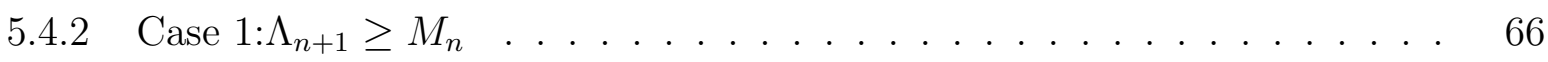

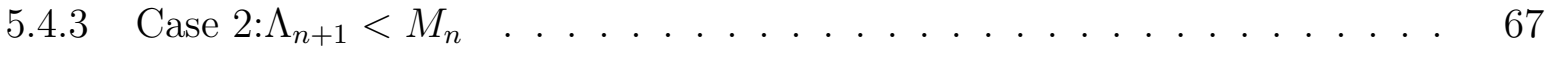

6 Conclusions and Future work $\quad 70$

6.1 Conclusion . . . . . . . . . . . . . . . . . . . . . . . . . . 70

6.2 Future Work . . . . . . . . . . . . . . . . . . . . . . . . 72 


\section{List of Tables}

$2.1 \mathrm{M} 2 \mathrm{M}$ applications and service requirements . . . . . . . . . . . . 7

2.2 M2M key architectural issues and solutions . . . . . . . . . . . . . . . . 11

3.1 Access classes and MTC UEs . . . . . . . . . . . . . . . . . . 23

3.2 Comparison of different congestion and overload control techniques . . . . . . 33

4.1 Simulation Parameters. . . . . . . . . . . . . . . . . . . 44

4.2 Simulation results of selecting probability . . . . . . . . . . . . . . 52

5.1 Channel bandwidth and resource block in LTE. . . . . . . . . . . . . . 57

5.2 Simulation Parameters for RARRA. . . . . . . . . . . . . . . 64 


\section{List of Figures}

1.1 Chapter Diagram . . . . . . . . . . . . . . . . . . . . . 4

2.1 An example of M2M communication network. . . . . . . . . . . . . . 6

2.2 M2M communication with LTE-A cellular network and possible congestion loca-

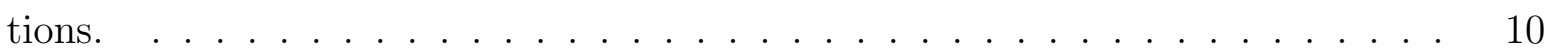

3.1 RAN based congestion control mechanisms. . . . . . . . . . . . . . . . . . 20

3.2 p-persistent random access method. . . . . . . . . . . . . . . . . . . . 21

3.3 Access class barring scheme. . . . . . . . . . . . . . . . . . . 24

3.4 Congestion control mechanisms applied to core network. . . . . . . . . . . . . 26

3.5 Extended access class barring scheme [1] . . . . . . . . . . . . . . . 27

3.6 Group based M2M communication. . . . . . . . . . . . . . . . . . . . . 30

3.7 Bulk handling in eNB $[2] . \ldots \ldots \ldots$. . . . . . . . . . . . . . . 31

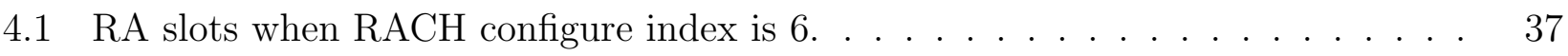

4.2 Performance analysis (total throughput) of slotted Aloha random access vs number of system load (both $\mathrm{H} 2 \mathrm{H}$ and $\mathrm{M} 2 \mathrm{M}$ users). . . . . . . . . . . . . . 38

4.3 Performance analysis (success probability and collision probability) of slotted Aloha random access vs number of system load (both $\mathrm{H} 2 \mathrm{H}$ and M2M users). . . 38

4.4 System model . . . . . . . . . . . . . . . . . . . . . . . . . . . . . . . . 39

4.5 Probabilistic Model . . . . . . . . . . . . . . . . . . . . . . . . . . . 40

4.6 System model for load balancing. . . . . . . . . . . . . . . . . . . . . . . 42

4.7 Flow chart of general ACB and proposed dynamic ACB method. . . . . . . . . 43 
4.8 Success probability of eNBs considering $\alpha_{1}=0.5, \alpha_{2}=0.8$ and $F T R_{1}=10$ and

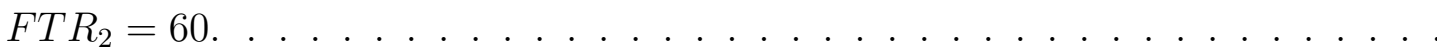

4.9 Throughput estimation of eNBs considering $\alpha_{1}=0.5, \alpha_{2}=0.8$ and $F T R_{1}=10$ and $F T R_{2}=60$.

4.10 Throughput and selection probability of eNBs when applying dynamic ACB with $F T R_{1}=F T R_{2} \ldots \ldots \ldots \ldots \ldots \ldots \ldots$

4.11 Throughput and selection probability of eNBs when applying dynamic ACB with $F T R_{1}>F T R_{2} \ldots \ldots \ldots \ldots \ldots$

4.12 Throughput and selection probability of eNBs when applying dynamic ACB with $F T R_{1}<F T R_{2} \ldots \ldots \ldots \ldots \ldots$

5.1 The LTE frame and radio resource block structure. . . . . . . . . . . . . . . 56

5.2 System model of relay assisted M2M communication. . . . . . . . . . . . . . . 58

5.3 Flow chart of radio resource allocation method. . . . . . . . . . . . . . . 62

5.4 Simulation model of relay assisted M2M communication. . . . . . . . . . . . . 63

5.5 Impact of buffer size when available bandwidth $W=20 \mathrm{MHz}$ and $L=30$. . . . 65

5.6 MTC channel capacity when $\Lambda_{n+1} \geq M_{n}, \tau^{t h} \doteq \frac{1}{L} \sum_{l=1}^{l=L} \gamma_{l}$, $=$ 50TTI and $W=20 \mathrm{MHz} . \ldots \ldots \ldots \ldots \ldots \ldots$

5.7 The MTC channel capacity with different threshold values. . . . . . . . . . . . . 68

5.8 Performance analysis of RARRA in term of sum throughput considering three different threshold values. . . . . . . . . . . . . . . . . . . 68

5.9 MTC channel capacity when $\Lambda_{n+1}<M_{n}, \aleph=30$ TTI and $W=20 \mathrm{MHz} . \quad$. . $\quad 69$ 


\section{List of Abbreviations}

M2M Machine-to-machine communication

MTC Machine-type communication

$\mathrm{H} 2 \mathrm{H} \quad$ Human-to-human communication

DACB Dynamic access class barring

RARRA Relay assisted radio resource allocation

UEs User equipments

MTCD Machine-type device

ACB Access class barring

RAN Radio access network

CN Core network

RA Random access

RACH Random access channel

PRACH Physical random access channel

RRC Radio resource connection

PDSCH Physical downlink shared channel

PUSCH Physical uplink shared channel

IMSI International mobile subscriber Identity

C-RNTI Cell radio network temporary identifier

USIM Universal subscriber identity module

SOOC Self-optimize overload control scheme

CAAC Congestion aware admission control method

CCE Control channel element

OFDM Orthogonal Frequency Division Multiplexing

SINR Signal to Interference and Noise Ratio

SNR Signal to Noise Ratio 


\section{Chapter 1}

\section{Introduction}

\subsection{Context of our study}

This thesis deals with two general problems of machine-to-machine (M2M) or machine-type communication (MTC) systems, referred to as massive access problem and radio resource allocation problem. Massive access problem becomes a major issue of MTC-enabled cellular networks because it creates congestion and overload problem in radio access network (RAN) and core network (CN) part of the backbone cellular network. In this thesis, we have proposed, implemented, and evaluated two solution strategies, namely dynamic access class barring method (DACB) and relay assisted radio resource allocation method (RARRA) to deal with these problems in a MTC-enabled cellular network. The main objective of the DACB method is to maximize the throughput of the base station while maintaining a fair distribution of all traffic (e.g. cellular and MTC) among base stations. Similarly, the main focus of the RARRA method is to maximize the sum throughput of the system by utilizing the available radio resource blocks and relay nodes to the MTC systems.

M2M (or MTC) communication is regarded as a new sophisticated technology that is emerging and will be integrated into the future cellular networks. Cellular networks such as LTE/LTEA and UMTS work as a backbone network to the MTC. The key difference between cellular communication and $\mathrm{M} 2 \mathrm{M}$ is that cellular networks support human-to-human $(\mathrm{H} 2 \mathrm{H})$ communication whereas MTC involves less human interaction. Besides this, the MTC devices are connected 
through MTC applications, e.g. smart meters, health care, smart grid etc. Each MTC application supports some distinguishable characteristic features such as low data rates, low mobility, infrequent transmission and massive number of low power autonomous devices connectivity. The diversity of MTC applications and its requirements impose new challenges in MTC-enabled cellular networks.

One of the major challenging issues is contention-based random access procedure which is designed for cellular networks, cannot accommodate a large number of M2M traffic. Contentionbased random access procedure such as slotted Aloha for cellular networks creates congestion when cellular networks support traffic from a large number of M2M devices. When a large number of M2M devices initiate random access procedure at a time, it will create preamble collision problem in physical random access channel (PRACH). There are a limited number of preamble sequences (i.e. 64 preamble for RACH configure index 6 [3]) available in each random access (RA) slot. Each device chooses one preamble sequence in a particular RA slot for contention. When more devices are activated at the same time and send contention requests to the base station (eNB), the eNB will get the same preamble sequence from more than one device. As a result, it will drop the conflicting requests and sends RA response to one device only. After a randomized backoff period, conflicting devices start the same RA procedure. This problem is regarded as massive access problem in cellular M2M networks.

Another challenging issue is radio resource allocation in cellular M2M systems in which M2M devices compete with $\mathrm{H} 2 \mathrm{H}$ users for the same radio resources. Compared to the $\mathrm{H} 2 \mathrm{H}$ users, M2M devices generate more uplink traffic. There are a very limited research works considering resource allocation problem in M2M/H2H co-existence scenarios. The authors in [4] [5] consider energy efficient and group based communication requirements in their resource allocation problem. In this thesis, we consider data rate maximization and relay allocation for the problem of resource allocation in cellular M2M systems which has not studied adequately before.

\subsection{Research problem}

This thesis addresses the massive access and radio resource allocation problems of machine-type communication systems. We have proposed a dynamic access class barring method taking into 
account the eNB selection and load balancing strategies to deal with massive access problem. For resource allocation problem, we have proposed a relay assisted radio resource allocation method with the aim to maximize the sum throughput of the system.

\subsection{Objective and contributions of the thesis}

The objectives of this thesis is two fold. In the first part, we address massive access problem and propose a access class barring method which is summarized as follows:

i) formulating an eNB selection method using maximum asposterior probability (MAP) criterion, where each device can choose lightly loaded base station,

ii) formulating an analytical model of load balancing which distributes M2M traffic among the base stations,

iii) proposing a dynamic ACB scheme which integrates both base station selection and load balancing method to handle massive access requests,

In the second part, we address radio resource allocation problem and provide solution for relay assisted allocation method for MTC system which is summarized as follows:

i) formulating an analytical model which estimates available resource blocks for M2M devices, ii) proposing a radio resource allocation method (RARRA) in which relay nodes help low SNR devices to send their access requests with the objective to improve system throughput.

\subsection{Thesis organization}

The organization of the thesis is shown in Figure 1.1.

Chapter 2 provides a general overview of machine-type communication (MTC) system in term of traffic characteristics, applications, features and service requirements. The key architectural issues of MTC enabled 3GPP networks, requirements and potential solutions are elaborately discussed here. Moreover, some challenging issues and research possibilities of MTC enabled cellular network are also discussed.

Chapter 3 presents a survey of massive access management methods for MTC enabled cellular networks. This chapter highlights the main distinguishable congestion control features of each 


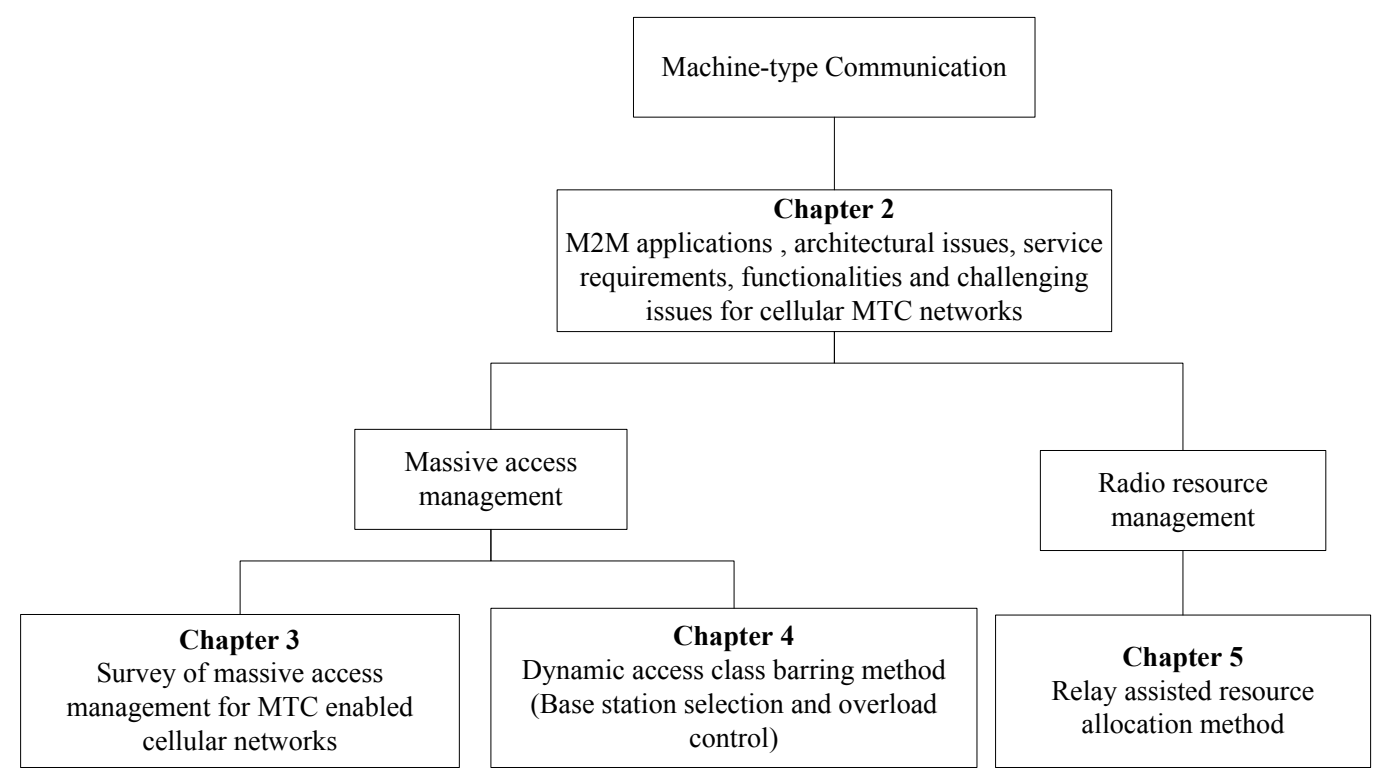

Figure 1.1: Chapter Diagram

solution and point out their limitations and areas for improvement. It also cover previous research works related to our research problems.

Chapter 4 presents an analysis of slotted Aloha random access procedure for M2M traffic in terms of throughput, access success probability and access collision probability. This chapter describes our proposed analytical solution for the massive access problem. A dynamic access class barring method to balance MTC traffic among base stations is discussed and evaluated in this chapter.

Chapter 5 presents a background study of radio resource allocation methods which are applicable for MTC systems and also describes our proposed relay assisted resource allocation method.

Chapter 6 summarizes the overall contributions of our thesis while discussing possible research directions. 


\section{Chapter 2}

\section{Machine-Type Communication}

In this chapter, we provide a general overview of machine-type communication (MTC) system. Firstly, we discuss MTC traffic characteristics, different type of MTC applications, features and service requirements. Then, the key architectural issues of MTC enabled 3GPP networks, requirements and potential solutions are elaborately discussed. Lastly, we highlight some challenging issues and research possibilities of MTC enabled cellular network. Machine type communication (MTC) is an emerging technology in communication system and networking research area. It is also referred to as a machine-to-machine (M2M) or device-to-device (D2D) communication. The key idea of MTC is that one or more machines communicate and network directly with one another without human intervention. M2M communications are done through MTC applications which support communication between machines and devices. These applications are widely adopted in our daily life and most of them are socially important. The applications and use cases of M2M communications are in power grid, health care, remote security surveillance, intelligent transport system, tracking and tracing etc.

These smart applications are aggregated to form the concept of smart city which focuses on applying next generation information technologies such as Internet of Things in our daily life. Internet of Things (IoTs), M2M communication and smart city applications, all of these are connected together mainly through wireless technologies. Progressive wireless technologies and traditional cellular communication networks are used as a backbone to the M2M networks. Figure 2.1 shows an example of M2M communication network where both uplink and downlink communication between MTC users and MTC devices are done through MTC application and 


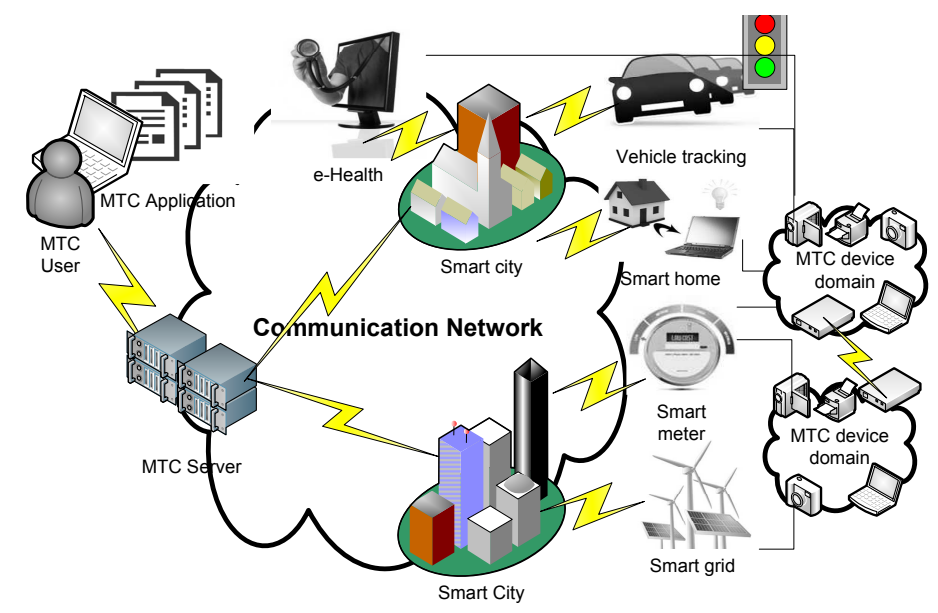

Figure 2.1: An example of M2M communication network.

communication network. These include applications ranging from e-Health to vehicle tracking and spanning over cities. Third Generation Partnership Project (3GPP) standardizes the infrastructure of M2M network in 3GPP networks (e.g., UMTS, LTE). Two working groups in 3GPP (3GPP service requirement working group and 3GPP system architecture working group) identify MTC features, service requirements and also present technical reports [3] [6] about architectural enhancement, addressing, triggering, charging and security issues etc. for machine type communication. 3GPP releases 10 and 11 [3] [7] specify the enhancement of system and radio access network (RAN) of 3GPP to support M2M traffic.

In this section, we provide some overview of machine-type-communication system. We present MTC features, its architecture over 3GPP network and identify MTC architectural requirements, its basic functionality and possible solutions.

\subsection{M2M applications and service requirements}

M2M communications have different service requirements and features which are different from non-M2M services, such as human-to-human communication, voice and data services. Authors in [6] [8] identify the following features for M2M communications:

- M2M traffic gives support to a very large number of devices communicating with each other at the same time. 
- Small data transmission: Each M2M device generates small amount of online or offline data and supports infrequent data traffic.

- Group based MTC features: Group based communication is needed for some applications, such as smart grid and fleet management.

- Low cost and low mobility: M2M traffic gives support to the low cost and low mobility devices.

- Time-controlled and time-tolerant: Only in a defined time frame, MTC devices can send and receive data.

- Power consumption of MTC devices is relatively low.

- Monitoring feature: MTC devices are used to monitor different applications specific data.

- Packet switched only: Packet switched services are required for MTC communications.

- Infrequent transmission and mobile originated communication are expected for MTC communication.

- Secure connection: Secure communication between MTC devices and MTC servers is expected for some M2M applications.

- Priority alarm: In case of vandalism and theft, MTC device with alarm feature has highest precedence.

Different M2M applications support different types of M2M devices and provide different services. An overview of these requirements are presented in Table 2.1.

Table 2.1: M2M applications and service requirements

\begin{tabular}{|l|l|l|l|}
\hline Service Area & M2M Application Area & \multicolumn{1}{|c|}{ Characteristics } & Service Requirements \\
\hline Security & -Surveillance systems & Small devices are used to monitor the & - Small data transmission \\
& -Car security & security system of the building and send & - Group based communi- \\
& -Building security & signal to central server in regular basis. & cations \\
& & & - Time sensitive \\
\hline
\end{tabular}

continued ... 


\begin{tabular}{|c|c|c|c|}
\hline Service Area & M2M Application Area & Characteristics & Service Requirements \\
\hline Metering & $\begin{array}{l}\text { - Power } \\
\text { - Gas } \\
\text { - Water } \\
\text { - Heating } \\
\text { - Smart grid } \\
\text { - Industrial metering }\end{array}$ & $\begin{array}{l}\text { Smart machines or smart meters are } \\
\text { known as M2M devices, gather utility } \\
\text { usage information from electrical appli- } \\
\text { ances. }\end{array}$ & $\begin{array}{l}\text { - Number of device hun- } \\
\text { dred to thousand } \\
\text { - Small data transmission } \\
\text { - Location specific trigger } \\
\text { - Low mobility }\end{array}$ \\
\hline Health & $\begin{array}{l}\text { - Health care for aged or } \\
\text { handicapped } \\
\text { - Remote diagnostics }\end{array}$ & $\begin{array}{l}\text { Some autonomous M2M devices mea- } \\
\text { sure patient's blood pressure, body } \\
\text { temperature, heart beat rate etc. at } \\
\text { regular intervals and simultaneously } \\
\text { send those information to the M2M } \\
\text { server in the hospital }\end{array}$ & $\begin{array}{l}\text { - Traffic flow is unidirec- } \\
\text { tional } \\
\text { - Small data transmission. }\end{array}$ \\
\hline $\begin{array}{l}\text { Tracking and } \\
\text { Tracing }\end{array}$ & $\begin{array}{l}\text { - Intelligent transport sys- } \\
\text { tem } \\
\text { - Fleet management } \\
\text { - Assest tracking } \\
\text { - Road tolling } \\
\text { - Traffic information }\end{array}$ & $\begin{array}{l}\text { Massive number of } \mathrm{M} 2 \mathrm{M} \text { devices are } \\
\text { used to support transport system. In } \\
\text { vehicle, M2M devices help to transmit } \\
\text { signal to each other. }\end{array}$ & $\begin{array}{l}\text { - Support massive number } \\
\text { of devices } \\
\text { - Low power consumption } \\
\text { - Group based communi- } \\
\text { cation. }\end{array}$ \\
\hline Payment & $\begin{array}{l}\text { - Point of sales } \\
\text { - Vending machines } \\
\text { - Gaming machines } \\
\text {-Pay for drive }\end{array}$ & $\begin{array}{l}\text { Some automated payment system and } \\
\text { vending machines use M2M devices to } \\
\text { send information in regular and secure } \\
\text { way. }\end{array}$ & $\begin{array}{l}\text { - Secure connection } \\
\text { - Time controlled } \\
\text { - Infrequent and small } \\
\text { data transmission }\end{array}$ \\
\hline $\begin{array}{l}\text { Remote } \\
\text { Maintenance }\end{array}$ & $\begin{array}{l}\text { - Sensors } \\
\text { - Elevetor control } \\
\text { - Vehicle diagnostics }\end{array}$ & $\begin{array}{l}\text { Some M2M devices act as a sensor and } \\
\text { controller to other devices. }\end{array}$ & $\begin{array}{l}\text { - Small data transmission } \\
\text { - MTC monitoring fea- } \\
\text { tures } \\
\text { - Low power and low mo- } \\
\text { bility }\end{array}$ \\
\hline
\end{tabular}




\section{$2.2 \quad$ M2M traffic characteristics}

Due to the diversity of M2M applications, M2M traffic shows different characteristics and random patterns, which differ M2M traffic from conventional cellular traffic. Authors in [9] [10] show some characteristics of M2M traffic; these are: i) time-controlled and delay-tolerant application specific traffic, ii) small amount of synchronize and periodic traffic, and iii) infrequent and oneway data traffic. In general, the M2M devices generate more uplink traffic than downlink as opposite to the existing cellular system. As the existing cellular system gives more emphasis on the downlink capacity rather than uplink, the emerging M2M enabled cellular systems need new protocols for allocating spectrum in the uplink layer. Authors in [10] present a hierarchical architecture for the cellular based M2M system. In this architecture, some devices (e.g. MTC gateway) work as a aggregate point, it combines M2M traffic and forwards the aggregated traffic to the upper layer. The overall description of the M2M enabled cellular architecture presents in the next subsection.

\subsection{M2M architecture underlaying 3GPP networks}

The system architecture working group (3GPP SA WG2) discussed various key architectural issues and proposed various solutions that were documented in [8,11,12]. Some architectural issues for machine type communication and proposed solutions are listed in Table 2.2.

The general architecture of M2M communication with LTE-Advanced (LTE-A) cellular network is shown in Figure 2.2, which includes the following components [6,13,14]. Three important domains related to MTC device, networking and application are shown with their interrelationships.

- M2M Device: Refers to as UEs (User Equipments), capable on capturing events and data. It can transmit and receive data among them and through the inter-connecting network.

- M2M Gateway: M2M gateway helps to inter-connect the MTC devices to the serving network. 


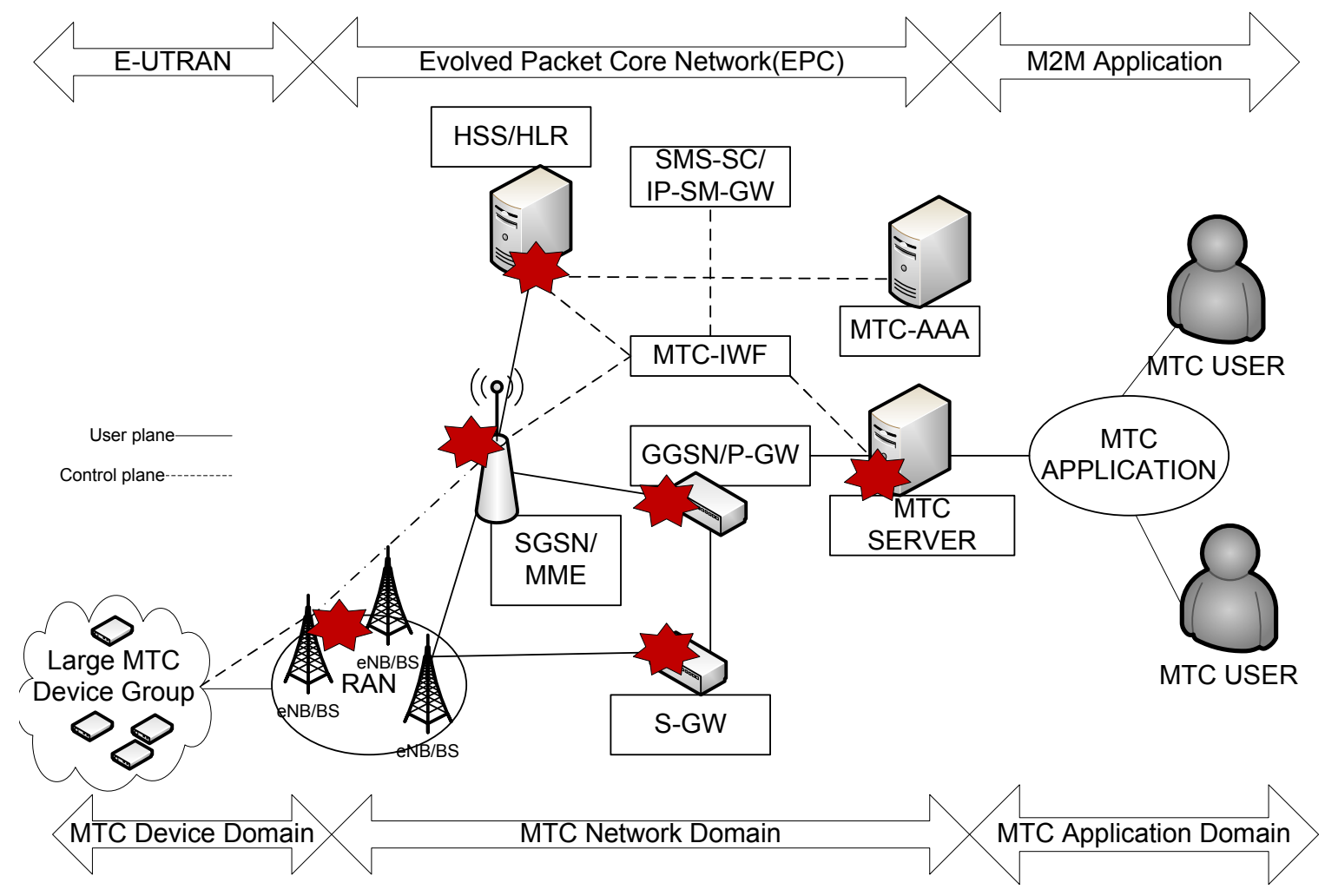

Figure 2.2: M2M communication with LTE-A cellular network and possible congestion locations.

- M2M Local Network: Group of M2M devices and M2M gateway serve as a M2M local area network.

- M2M Communication Network: M2M network domain provides communication between M2M gateway and M2M application. LTE is the example of communication network and its main elements are:

- eNBs: eNodeBs refers to evolved RAN (Radio Access Network), a sophisticated version of LTE base station.

- MME/SGSN: Mobility management entity controls the bearer activation/deactivation process in LTE access network and it assumes functions related to attachment and connection management. It controls user authentication procedure across the network.

- P-GW/S-GW: Packet data node gateway works as an interface between external packet data and LTE network. Serving gateway receives and transmits user data 
packets and also controls inter handover among eNodeBs. It works as an interface between 3G and LTE network.

- HSS: The home subscriber server contains routing and subscription related information of users. It also holds subscriber location and identities information of users. It determines when a service capability server (SCS) performs device trigger to a particular MTC device.

- MTC-IWF: MTC interworking function unit maintains communication between the 3GPP networks and the SCS. It provides support to one or more SCS and invokes specific functionalities for SCS such as authentication, device triggering request and response etc.

- MTC-AAA: MTC AAA server works with HSS to provide identification information of MTC device. It translates external identifier corresponding to an international mobile subscriber identity (IMSI) and also helps to replace IMSI with an external identifier.

- M2M Application: M2M application serves as an user interface in which users can give commands to specific M2M devices. It is also considered as a destination of data sent by a device over the network. MTC server, MTC user and MTC client application together serve as a specific business processing engine.

Table 2.2: M2M key architectural issues and solutions

\begin{tabular}{|l|c|c|}
\hline Architectural Issues & \multicolumn{1}{|c|}{ Required Functionality } & \multicolumn{1}{c|}{ Proposed Solutions } \\
\hline IP addressing & -IPv4 address space is insufficient for & - Due to massive number of MTC device, IPV4 \\
mass amounts of MTC UEs. & addresses are not sufficient for addressing. \\
& & ing MTC devices. IPv6 address mechanisms \\
& are considered as a primary method for ad- \\
& dressing to MTC devices. \\
\hline
\end{tabular}

continued ... 


\begin{tabular}{|c|c|c|}
\hline Architectural Issues & Required Functionality & Proposed Solutions \\
\hline $\begin{array}{l}\text { Online and offline } \\
\text { small data trans- } \\
\text { mission }\end{array}$ & $\begin{array}{l}\text {-It is required to accommodate small } \\
\text { data transmission over } 3 \mathrm{GPP} \text { networks. } \\
\text {-Online/offline small data transmission } \\
\text { mechanism needs to be defined by net- } \\
\text { work operator policy. }\end{array}$ & $\begin{array}{l}\text {-MTC devices with online and offline small } \\
\text { data transmission use SMS system via } \\
\text { SGSN/MSC or SMS over SGs [3]. }\end{array}$ \\
\hline MTC device trigger & $\begin{array}{l}\text {-Triggering of MTC devices needs iden- } \\
\text { tifier to identify MTC device. } \\
\text {-It is required to define different trig- } \\
\text { gering mechanisms for MTC device and } \\
\text { MTC server. } \\
\text {-The trigger mechanism needs to de- } \\
\text { fine a scalable transmission procedure } \\
\text { to send and receive trigger request, re- } \\
\text { sponse messages among multiple MTC } \\
\text { devices. }\end{array}$ & $\begin{array}{l}\text {-MTC device trigger is a service presented by } \\
\text { the } 3 \mathrm{GPP} \text { system for the MTC server over } \\
\text { control plane signaling. } \\
\text { - MTC application level triggering activity } \\
\text { does not consider as a device trigger in } 3 \mathrm{GPP} \\
\text { system, it requires a poll model for communi- } \\
\text { cations between MTC devices and the MTC } \\
\text { servers. } \\
\text {-Device triggering, communication between } \\
\text { packet data networks and M2M networks are } \\
\text { shown in [16]. }\end{array}$ \\
\hline MTC subscriptions & $\begin{array}{l}\text { - MTC subscriber needs to activate and } \\
\text { deactivate several MTC features on a } \\
\text { MTC device. } \\
\text { - It is required for a network operator } \\
\text { to restrict incompatible MTC features } \\
\text { subscription according to network oper- } \\
\text { ator policy. }\end{array}$ & $\begin{array}{l}\text {-The activation/deactivation of MTC features } \\
\text { could be done by MTC subscriber in a regular } \\
\text { interval via web interface. } \\
\text {-MTC subscriber associate each MTC device } \\
\text { with one MTC subscription by including au- } \\
\text { thentication information. }\end{array}$ \\
\hline MTC identifiers & $\begin{array}{l}\text { - M2M needs identifiers up to two or- } \\
\text { ders of magnitude higher than } \mathrm{H} 2 \mathrm{H} \\
\text { communication. } \\
\text { - It is required to design globally unique } \\
\text { external identifier for identifying UEs } \\
\text { used for MTC. }\end{array}$ & $\begin{array}{l}\text {-3GPP defines external identifier which is used } \\
\text { by the M2M network to identify the MTC sub- } \\
\text { scription. } \\
\text { - Each MTC UE has an International Mobile } \\
\text { Subscriber Identity (IMSI). } \\
\text { - Universal Subscriber Identity Module } \\
\text { (USIM) in the MTC device helps to bind each } \\
\text { IMSI with the individual features in the home } \\
\text { subscriber server (HSS). }\end{array}$ \\
\hline
\end{tabular}

continued ... 


\begin{tabular}{|c|c|c|}
\hline Architectural Issues & Required Functionality & Proposed Solutions \\
\hline $\begin{array}{l}\text { Signaling conges- } \\
\text { tion control }\end{array}$ & $\begin{array}{l}\text {-It is required to design an effective } \\
\text { mechanism for handling MTC related } \\
\text { signalling congestion and overload. } \\
\text {-It is required to reduce signalling load } \\
\text { such as attach, PDP/PDN activation, } \\
\text { service request etc. from MTC devices } \\
\text { belonging to particular MTC group. } \\
\text { - In order to avoid network congestion, } \\
\text { it is required to measure how frequently } \\
\text { a particular MTC group/application } \\
\text { generates connection request. }\end{array}$ & $\begin{array}{l}\text {-Signalling congestion can be handled by ap- } \\
\text { plying variable time backoff schema [17], ac- } \\
\text { cess class bearing scheme [18], [19], PRACH } \\
\text { resource sharing schema etc. } \\
\text {-3GPP provides three solutions to control } \\
\text { overload: i) Broadcasting MTC access con- } \\
\text { trol by RAN. iii) The eNodeB/RNC/BSS re- } \\
\text { ject RRC connection and channel request. i) } \\
\text { If congestion situation occurs in the core part } \\
\text { of the network, the SGSN/MME point reject } \\
\text { extra connection requests [7] [20]. }\end{array}$ \\
\hline $\begin{array}{l}\text { Decoupling } \mathrm{MTC} \\
\text { server from } 3 \mathrm{GPP} \\
\text { architecture }\end{array}$ & $\begin{array}{l}\text { - MTC server may be deployed outside } \\
\text { of the mobile network. } \\
\text { - It is required to design an interface for } \\
\text { secure communications between MTC } \\
\text { service logic components and } 3 \mathrm{GPP} \\
\text { PLMN. }\end{array}$ & $\begin{array}{l}3 \mathrm{GPP} \text { MNO can restrict to access informa- } \\
\text { tion, resources and services in the core net- } \\
\text { works by using Service Abstraction Layer. }\end{array}$ \\
\hline Time Controlled & $\begin{array}{l}\text {-Time controlled MTC feature requires } \\
\text { a mechanism to allocate time periods } \\
\text { for a group of MTC devices. }\end{array}$ & $\begin{array}{l}\text { - The } 3 \text { GPP system defined two time inter- } \\
\text { vals, grant time and forbidden time interval } \\
\text { for controlling communication with the MTC } \\
\text { users and MTC servers. }\end{array}$ \\
\hline
\end{tabular}

\subsection{Challenging issues of M2M enable cellular networks}

High speed transmission and large volume of data are the general requirements of $\mathrm{H} 2 \mathrm{H}$ traffic whereas M2M requires small data transmission, group-based, time-controlled and delay-tolerant transmission. Aggregation of these two traffic becomes more challenging in terms of new architecture design, air interface consideration, radio resource management etc. Device identification, subscribing and unsubscribing of distinct MTC features and device triggering become more critical problems when communication is done through the legacy cellular networks. Although $3 \mathrm{GPP}$ takes initiative to resolve those problems, more research work is still needed to improve the use 
of M2M networks. Researchers should address the following factors among others:

- Massive access management issue: The key features of M2M networks are massive number of devices, massive connectivity and explosive traffic. Massive access management is one of the main challenging problems of M2M enabled cellular networks as devices are scattered all over the network and M2M device density is higher than cellular device.

- Power management issue: Most of the MTC devices are battery-powered equipment and hence have limited life time. Sometimes remotely controlled devices are unable to change their power sources. For example, ecological network (EcoNet) [21] was developed for monitoring and tracing animals biological behaviours. In this network, inter and intra species interaction was observed by smart devices (e.g., GPS, sensors). Efficiency of this network depends on the durability of those devices. Minimum power consumption and energy saving become challenging issues in M2M networks.

- Radio resource management and scheduling: Diversity of applications, various features and requirements of M2M networks become more critical when communication is done through cellular networks. Resource management, sharing, scheduling and prioritization techniques need to be addressed properly.

- Self organization, self management and configuration techniques: These are important factors in M2M networks as it can work without human involvement. Due to the expected higher number of MTC connections, the autonomous operations in terms of reconfiguration and redundant connectivity in case of any disasters should be incorporated in the MTC networking design.

- Group based communication issue: When M2M networks support group communication features, proper coordinator selection and group formation become an another important factor that needs to be considered.

- Security related issue: Secure connection and quality of service are two main important issues in M2M based biomedical networks which support e-Heath and carry extremely important life saving and sensitive data. 
- Prioritizing methods: Another challenging issue is to design priority mechanisms to handle voice and emergency service in M2M networks during congestion situation giving rise to QoS for real-time applications where multimedia M2M applications are deployed, for example intruder detection followed by voice communication and video recording/transmission.

In the next chapter, we will discuss in details the massive access problem in cellular machinetype system and present a comparative analysis of among different solution methods. 


\section{Chapter 3}

\section{Survey of Massive Access Management Methods for MTC enabled Cellular Networks}

In this chapter, we discuss massive access management solutions, present a detailed description of each solution technique, classify them in two categories from a massive access and congestion control. We also highlight the main distinguishable congestion control features of each solution and identify their limitations and areas for improvement.

\subsection{Congestion in MTC over 3GPP Network}

One of the key challenging issues of M2M communication is to accommodate massive amount of M2M traffic in traditional cellular networks. Massive access characteristics of M2M network create overload problem in cellular networks. 3GPP technical report [3] [22] identifies three reasons that create MTC related signalling congestion: i) erroneous MTC applications or MTC server activate a lot of MTC devices at a time, ii) a large number of MTC devices send connection/activation/modify requests at the same time, and iii) recurring applications such as reporting sensed data in regular intervals which are activated in certain time. One of the main challenging problems is to distinguish MTC traffic from non-MTC traffic and to block the traffic of malfunctioning applications. Rejecting attach request or connection request, back-off 
time and access-class barring (ACB) method, broadcasting MTC access control by RAN have been studied in 3GPP. The improved version of ACB congestion control methods are extended ACB [19], prioritized ACB [23], adaptive ACB [18], cooperative ACB [24] which have been proposed by several researchers. Survey of these techniques have been presented in [25] [26] where authors analysed these techniques in terms of performance metrics such as success rate, energy consumption and delay. Radio resource allocation problem, overload problem and different scheduling techniques for M2M users have been analyzed in [27], where different techniques have been proposed to allocate radio resources for MTC. application specific congestion control is an on-going research activity in $3 \mathrm{GPP}$ [28]. To the best of our knowledge, there are only a few survey articles available in M2M networks that investigate congestion problem and its solutions. In this section, firstly, we present the main reasons of signalling congestion problem that create overload in communication network. We analyse this problem scenario in radio access network and core network point of view. In the next section, we present a comprehensive survey of different overload control methods that are applied both radio access network (RAN) and core network $(\mathrm{CN})$ part of the network.

\subsubsection{Signalling congestion scenarios}

Congestion scenarios occur in the signalling network when the massive number of MTC devices send attachment or activation requests to the network at the same time. In M2M communication, congestion and overload in the signalling network are caused by the following four reasons: (1) massive number of attachment requests to the network simultaneously,

(2) send and receive a high volume of activate/modify/deactivate connection requests at the same time,

(3) malfunctioning in the MTC application or MTC server,

(4) recurrent applications that are synchronized to the predefined time (half/quater hour).

In a M2M system, some applications such as remote surveillance and monitoring system generate simultaneous data at periodic time intervals (e.g., periodically every hour or half hour) and consequently create overload situation in that time interval. When a massive number of connec- 
tion/activation requests come from significantly large area in the same time interval, it creates congestion situation in both radio access part (eNB/BS) and core network part (MME/SGSN) in the serving network. Figure 2.2 showed the main point of signaling congestion area in M2M networks. According to [29] due to the massive number of concurrent access of M2M devices, the network becomes overloaded in the following area.

\subsubsection{Overload situation in radio access network (RAN)}

Radio network becomes overloaded for a certain period of time because of the huge number of concurrent data exchange among some MTC applications. Massive amount of concurrent data transmissions by MTC sensors at the same location at the same time overload the radio network. RAN level congestion occurs in eNB/BS location due to random access procedure.

- Congestion and overload situation in random-access procedure: Random access procedure is a process that is initiated by idle UE (mobile terminal) that attempts to connect to the LTE network. Contention based random access (RA) comprises of four steps which are as follows:

Step 1-Select RA preamble sequence: The MTC UE sends a randomly selected RA preamble sequence on Physical Random Access Channel (PRACH) to the eNB (base station). Step 2-Receive RA response: In the second step, the base station transmits RA response on the Physical Downlink Shared Channel (PDSCH) in response to the detected preamble sequence.

Step 3-RRC connection request: The MTC UE sends RRC connection request to base station using Physical Uplink Shared Channel (PUSCH). RRC connection request message includes temporary C-RNTI (Cell Radio Network Temporary Identifier) and scheduling information.

Step 4-Acknowledge RRC connection: The base station (eNB) sends RRC connection setup message to the MTC UE. In this step, the base station echoes the UE identity it received in the third step on PDSCH.

In a LTE-A network, the UE performs random access (RA) when it changes its stage from 
sleep or idle mode to active mode. There are 64 preamble sequences available in each cell in a LTE-A network. Each UE randomly selects one preamble sequence for access the RACH channel. A collision occurs when more than two UEs randomly select the same preamble sequence. Overload and congestion scenarios occur in RAN when massive amount off MTC UEs attempt to access LTE-A's base station at the same time.

\subsubsection{Core Network (CN) congestion scenario:}

Core network becomes congested when a MTC user activates a large group of MTC devices using many MTC applications, and it will cause a core network overload. Normally, the group of MTC devices are located in different parts of the network. When these devices are activated,

they constantly propagate their signalling message into core network. Figure 2.2 shows that the serving gateway (SGSN) and MTC server become affected due to congestion in core network.

\subsubsection{Congestion control methods applied to RAN}

There are various random access channel overload control mechanisms proposed for 3GPP networks. Some new techniques to control overload for massive access in machine type communication are categorized in Figure 3.1. Push-based method and pull-based method are the two main category of congestion control mechanisms applied to RAN. Push-based method mainly focus on contention based random access procedure whereas pull based method use for contention free procedure. These two mechanisms are further categorized according to the channel information, RAN resources (e.g. frames, time interval etc.), RAN elements (e.g., eNB ) and RA elements (e.g. RA-slots, preambles etc.). Overview of these mechanisms are discussed below:

1. Push-based method: In push-based mechanisms, each MTC device initiates random access procedure and uses different methods to control overload in random access channel. Four subclass of push-based mechanisms are described here.

(a) Randomized Access Control Method:

- Backoff Timer Method: In the backoff timer method, the MTC devices set a large value of backoff time and RACH overload is controlled by delaying RA attempts for 


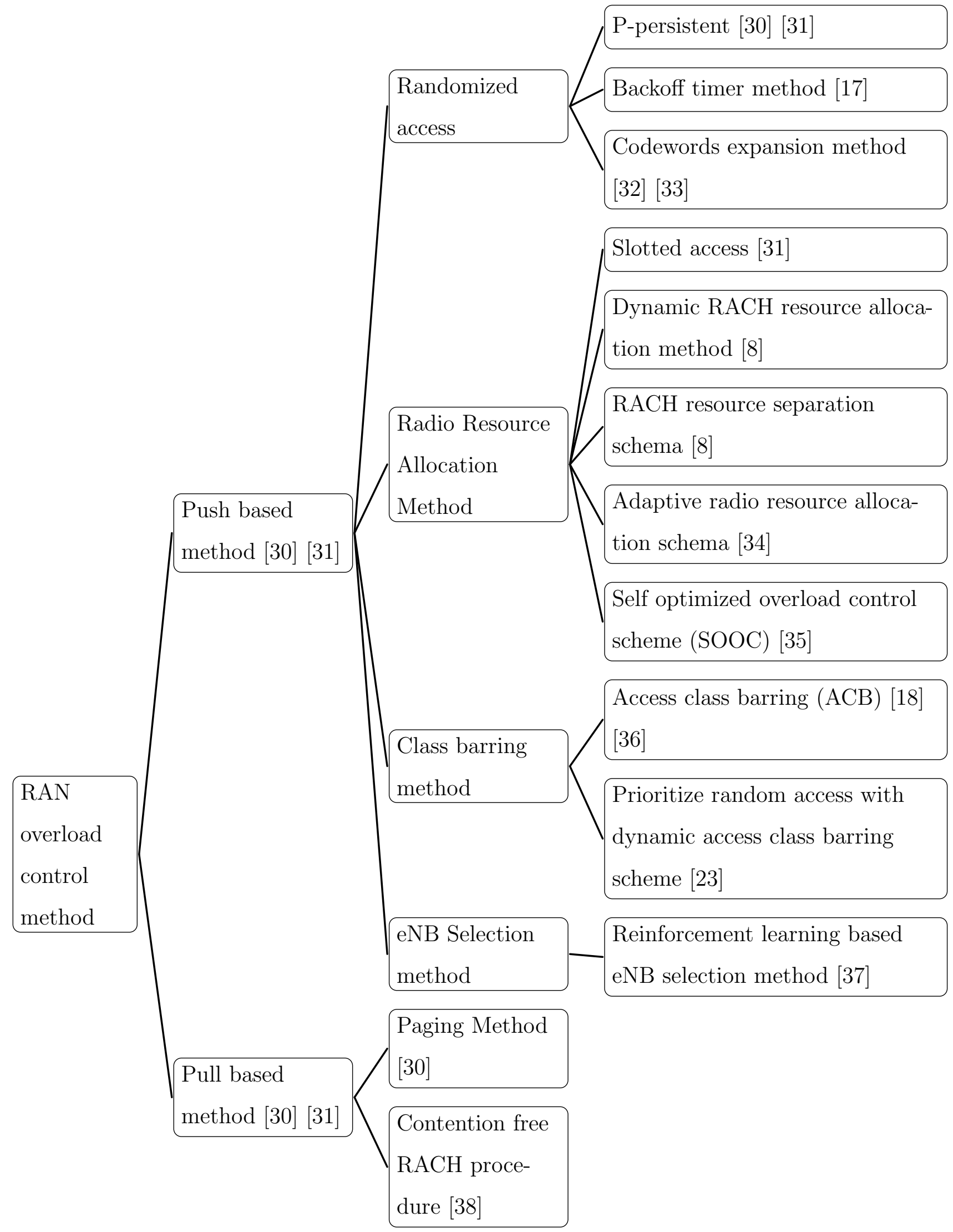

Figure 3.1: RAN based congestion control mechanisms. 


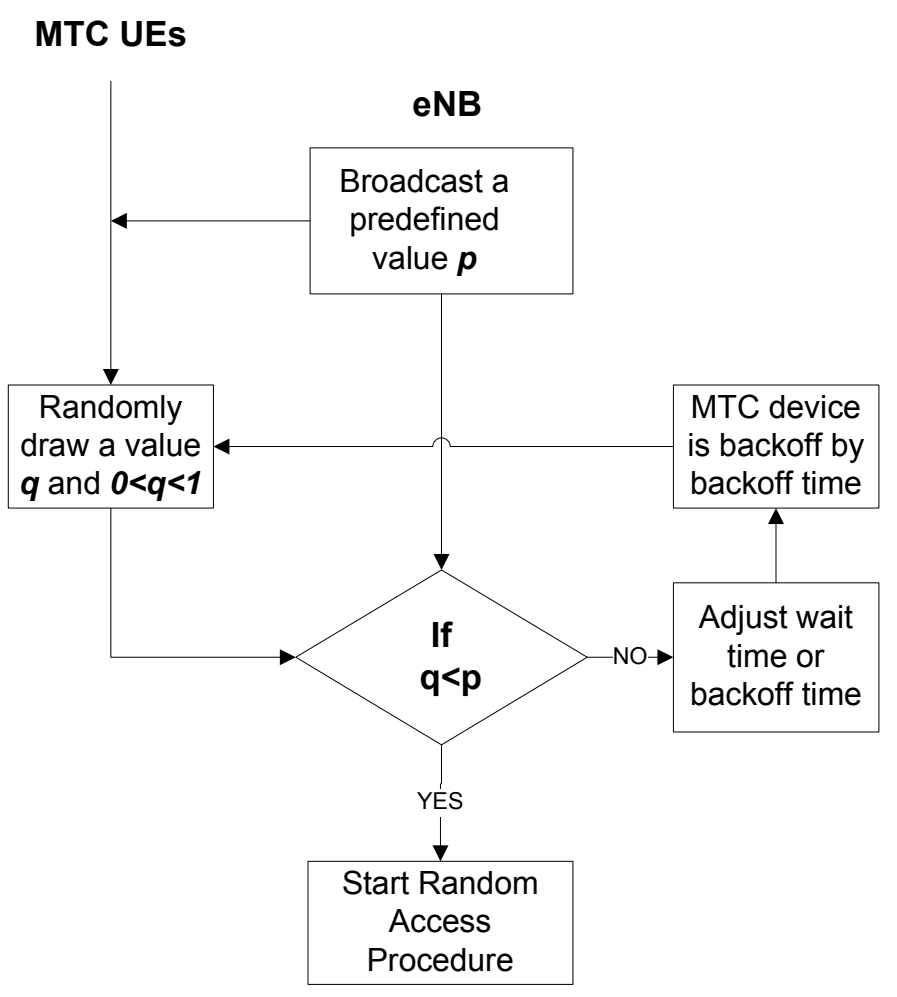

Figure 3.2: $p$-persistent random access method.

MTC devices and UEs separately. In [17] [39], it is shown that both MTC devices and UEs in 3GPP create continuous collisions at the random access channel (RACH) when a large number of MTC devices and UEs attempt to access RACH simultaneously. Backoff scheme works well in low load situation but it is less effective under high congestion level.

- p-persistent: Figure 3.2 depicts the p-persistent random access method. In this method, a predefined value $p$ is assigned to each MTC device and MTC device selects a random number between 0 and 1 . If this number is less then the device's $p$-persistent value, it can start random access; otherwise, it will backoff and wait for next interval. Backoff time and wait time among RA preambles selection and receiving RA response message, RRC connection request and response message can be set according to the PRACH channel condition.

- Codewords Expansion Method: Random access codeword for machine-type communication was proposed in [33]. Authors proposed a virtual frame structure for RA 
and each virtual frame consists of multiple random access sub-frames. In this codeexpanded method, each UE selects random codewords per every sub-frame of the virtual frame but in the ordinary scheme, each UE selects only one preamble for one of the sub-frames of the virtual frame. This codeword expansion method enhances contention resources such as sub-frame and preamble sequence. The efficiency of this method is estimated as $S_{e}, S_{e}=\frac{N_{s}}{N_{p}}$ where $N_{s}$ is the expected number of single codewords and $N_{p}$ is the expected number of all available codewords and authors designed Markov chain model to calculate $N_{p}$.

(b) Radio Resource Allocation Method:

-Slotted-Access Scheme: In this scheme, specific RA-slots within specific radio frames are allocated to each M2M terminal for preamble transmission. The mechanism of access to RA-slots is done within a RA cycle. The base station broadcasts the RA cycle which is an integer multiple of radio frames. The RA cycle length is proportional to the number of unique RA-slots within a radio frame. If the number of M2M terminals is greater than the total number of unique RA-slots, overload will occur in random access channel. The same RA-slots are shared by multiple MTC devices and consequently preamble collisions will occur in PRACH. In [7], it is shown that each MTC device is associated with an access slot through its ID (IMSI) and preamble collisions can be reduced by setting longer RA cycle.

-PRACH Resource Separation Scheme: In this scheme, PRACH resources such as preamble sequences and RA slots are separately allocated to M2M terminals and $\mathrm{H} 2 \mathrm{H}$ terminals. If the the M2M terminals share the same PRACH resources as $\mathrm{H} 2 \mathrm{H}$ terminals, then PRACH overload condition could significantly affect RA quality of $\mathrm{H} 2 \mathrm{H}$ terminals.

-Dynamic PRACH Resource Allocation Scheme: The base station dynamically allocates RA slots based on the PRACH load condition. The base station runs an algorithm based on the high load conditions and dynamically allocates RACH resources to MTC devices.

-Adaptive Radio Resource Allocation Method: Although resource separation scheme 
assigns different resource block (RB) to M2M and H2H user, adaptive resource allocation method dynamically adjusts RBs based on the available and reserved signaling RB. Reserved set of RB is used for rejection message signaling purpose. The objective of this mechanism is to maximize the adaptive parameter $e$,

$$
e=\text { Available RB }- \text { Use RB for MTC }+ \text { Reserved RB }
$$

so that $\mathrm{H} 2 \mathrm{H}$ user gets enough RB. Authors in [34] simulate this mechanism with the aim to improve throughput. This method also mitigates RAN level congestion which occurs between MTC device and eNB.

-Self-optimize Overload Control Scheme (SOOC): Authors in [35] proposed a selfoptimizing overload control mechanism which can respond to suddenly changing PRACH load conditions in a timely manner. In a SOOC mechanism, the base station adds or reduces PRACH resources when it detects an increase or decrease in PRACH load by implementing intelligent control loop. SOOC includes a composite of overload mechanism which comprises of dynamic PRACH resource allocation, PRACH resource separation, the ACB scheme, the slotted-access scheme, and the $p$-persistent scheme.

(c) Class Barring Method: -Access Class Barring (ACB) Scheme: Figure 3.3 shows the access class barring scheme for random access procedure. The general procedure of ACB scheme and $p$-persistent is same but ACB scheme categorizes UEs into 16 access classes (ACs). Table 3.1 shows the category of MTC users and their corresponding $\mathrm{AC}$ number.

Table 3.1: Access classes and MTC UEs

\begin{tabular}{|c|c|}
\hline Access Class No & MTC Users \\
\hline $0-9$ & Normal UEs \\
\hline 10 & Emergency services/Emergency call \\
\hline $11-16$ & Higher Priority UEs \\
\hline
\end{tabular}

Figure 3.3 shows that at first each eNB broadcasts acBarringFactor $p$ and sets acBarringTime value, and each MTC UE selects a random value from 0 to 16 . If 
this value is less than acBarringFactor, the MTC UE starts the random access procedure. Otherwise, the MTC UE is backed off by an acBarringTime duration. The ACB-based scheme is a congestion avoidance technique in the RACH by blocking MTC devices with a small value of $p$. In general, the ACB scheme deals with excessive PRACH overload by reducing the number of M2M attempting RAs.

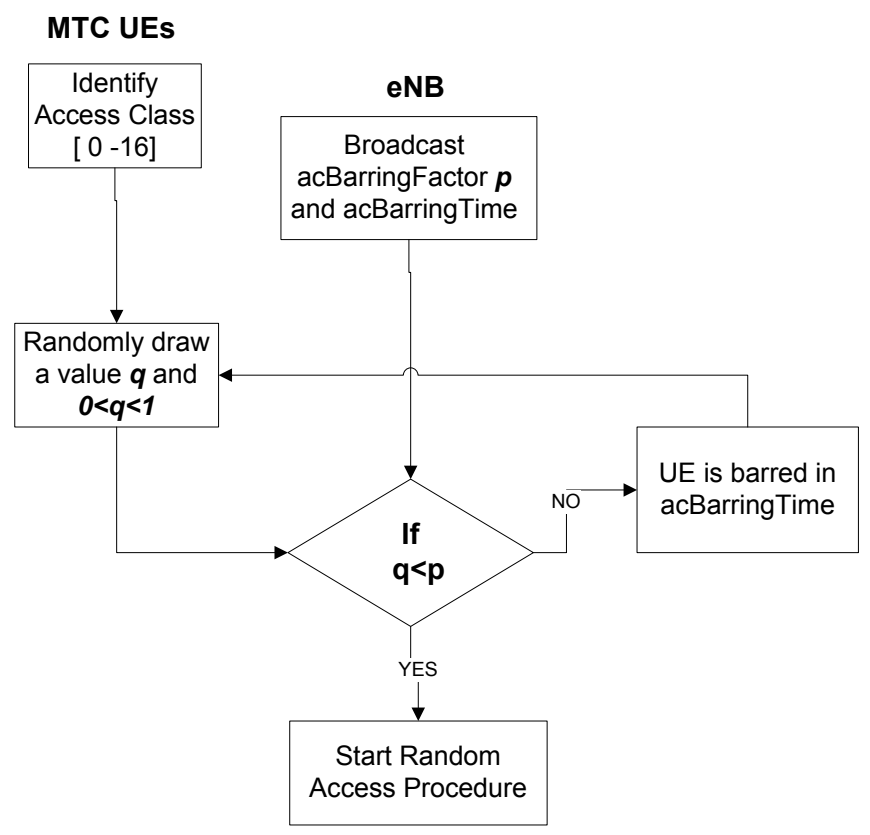

Figure 3.3: Access class barring scheme.

Authors in [36] compared between no barring and access class barring methods. They define two performance metrics: access success probability and average access delay which refers to average time between the first access attempt by the UE to the successful end of random access procedure. Simulation results showed that without barring control, the total number of preamble transmission is six times higher than the total number of preambles available, and access success probability is unacceptable. In case of $\mathrm{ACB}$, the success probability depends on acBarringFactor and acBarringTime. Success probability shows higher value when acBarringFactor value becomes low and acBarringTime is high.

-Prioritized Random Access with Dynamic ACB Scheme: Authors in [23] proposed prioritized RA mechanisms which use dynamic access barring scheme. In this scheme, different MTC classes use pre-allocating RACH resources and set their back-off timer 
and procedure according to their classes priority.

(d) eNB Selection Method

- Reinforcement learning based eNB selection method: A MTC device selects an eNB based on the information of relative signal strength and available channel gain. Authors in [37] proposed reinforcement learning based eNB selection method for those MTC devices which reside into a coverage area of more than one eNB. The MTC device can select, adapt and observe eNB based on the QoS parameters such as packet transmission time. Reinforcement eNB selection method ensures QoS and balances network load based on the learning process called explanation step where the objective is to maintain minimize packet delay time by selecting appropriate eNB.

2. Pull-based Scheme: It is a centralized RAN overload control scheme. In this method, network node such as eNBs or base station initiate RACH procedure upon receiving a request from MTC server. Paging and contention free RACH procedure work similar to a pulling method.

- Paging method: M2M server triggers LTE's base station to page the intended M2M terminals. When paging signal is received, the M2M terminal will initiate RA. The base station can control the number of M2M terminals by observing the PRACH load condition and available resource condition. Paging method requires additional control channel resource for paging massive number of MTC devices.

- Contention free RACH procedure: eNB starts contention free RACH procedure by sending dedicated preamble sequence to the MTC device. MTC device initiates RACH procedure using the assigned preamble sequence. After receiving the assigned preamble, eNB sends random access response within random access response (RAR) window. 3 GPP [22] [38] specifies both contention free and contention based procedure where former procedure needs reserved RACH resources for initialization of random access. 


\subsubsection{Congestion control methods applied to CN}

Core network congestion situation arises when group based communication occurs. Usually MTC server or MME creates congestion in the core network when a large number of MTC devices, belonging to the same MTC group send and receive data simultaneously. Figure 3.4 shows various congestion control mechanisms which are applicable to mitigate congestion in core network.

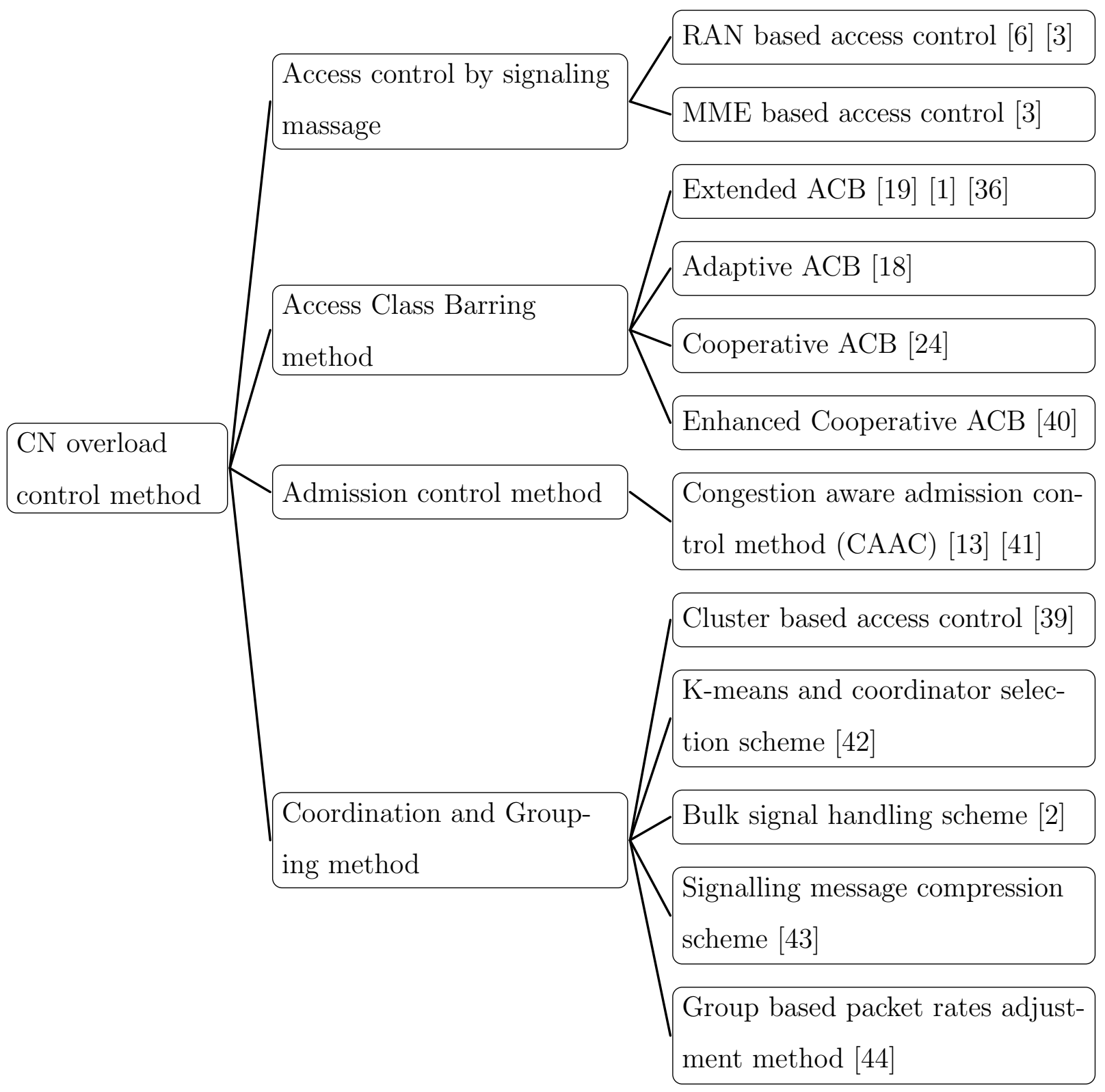

Figure 3.4: Congestion control mechanisms applied to core network. 
1. Access Class Barring method:

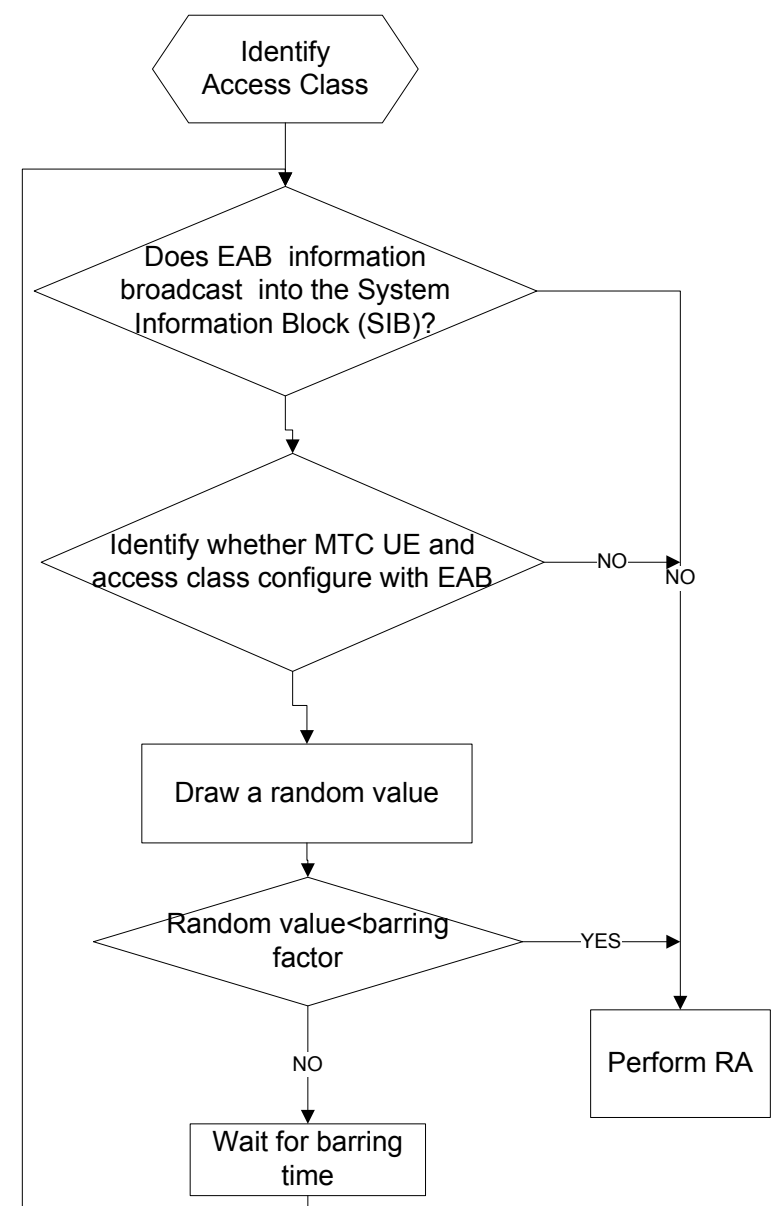

Figure 3.5: Extended access class barring scheme [1].

- Extended ACB: Extended access barring mechanisms alleviate the barring factor and barring time problem of ACB which are common for ACs 0-9. Also there is no quality of service in terms of barring probabilities for access class of 0 to 9 . Figure 3.5 shows the details of EAB mechanism. System information block (SIB) includes the EAB barring factor and barring time information which are broadcast by eNB. Only a subgroup of MTC UEs receives this information, so that collision among themselves is manageable. MTC devices whose EAB were enabled, draw a random value ranging from zero to one and compare it with the EAB barring factor. If the value is smaller than the barring factor, device is allowed to perform random access. Otherwise, MTC device is barred until EAB barring time. One of the key differences between EAB 
and $\mathrm{ACB}$ is that in $\mathrm{EAB}$, the $\mathrm{ACs}$ are either completely barred or unbarred; but in $\mathrm{ACB}$ it is based on probability. In ACB, among the MTC UEs belonging to the same $\mathrm{AC}$, some UEs are barred while others are not whereas in EAB, UEs belong to the same group are either completely barred or unbarred.

Authors in [1] proposed an algorithm to determine a barring factor of EAB which depends on predicting the future RA attempt number $P(t)$ and upper bound constraint on new RA attempt number $P_{\text {bound }}(t)$. Once eNB decides the barring factor $B F(t)=P_{\text {bound }}(t) / P(t)$, it will send paging message to inform MTC devices. Their proposed solution helps to reduce average access latency and also reduces the number of barred MTC devices. In [19], authors provide a detailed analysis of EAB mechanism that works on 3GPP LTE-A network to control the access load. The admission rate was derived using $\mathrm{EAB}$ that depends on barring time and paging cycles.

- Adaptive ACB Scheme: In [18], authors proposed adaptive ACB parameters by which eNBs adjust the access probability $p$ and barring duration based on system load at regular intervals. In high load situations, the access probability and access barring duration are adaptively increased whereas in low load conditions, these values become decreased. The system information block (SIB) which carries random access channel information periodically updates the ACB parameters. The network simulation in [18] shows that adaptive ACB improves system performance in high load condition and shows better performance among other fixed ACB and back off time scheme.

- Cooperative Access Class Barring Scheme: In [24] authors propose a cooperative ACB scheme for machine type communication. Cooperative ACB modifies the ordinary ACB parameter $p$ based on the joint decision by all base stations (BSs). The access load sharing and stabilization are ensured globally among all BSs in cooperative ACB scheme whereas in ACB schema, each eNB individually decides the value of the parameter $p$.

- Enhanced Cooperative Access Class Barring Scheme: In cooperative access class barring scheme, all eNBs jointly decide ACB parameter $P_{n}=\left\{P_{1} \ldots \ldots . . P_{M}\right\}$ where $M$ is the total number of eNBs. When a MTC device receives $P_{n}$, the $n$th MTC device 
estimates access probability, $Q_{n, i}=\frac{P_{i}}{\sum_{x \in M} P_{x}}$ for selecting $i$ th eNB. In [40] authors propose an enhanced cooperative access class barring scheme which modifies MTC access probability by considering the number of attached MTC devices. The modified MTC access probability is $Q_{n, i}=\frac{P_{i}}{\sum_{x \in M} P_{x}}\left(\frac{1}{\left\|A_{i}\right\|+1}\right)$, where $A_{i}$ denotes the set of MTC devices attached to the $i$ th eNB and $\left\|A_{i}\right\|$ is the norm of $A_{i}$. Enhanced cooperative ACB scheme shows better improvement in throughput and also reduce MTC access delay than cooperative ACB.

2. Access control by signaling massage

- RAN-based Access Control: RAN node such as eNB/RNC/BSS can be used to control the overload. The randomized barring factor computed as $T=(0.7+0.6 \times$ rand $) \times$ acBarringTime is used to control the network access. RAN node adjusts the barring information when the number of MTC devices RA attempt increase significantly. MTC baring information contains barring factor, barring time, MTC group to block etc.

- MME/SGSN based Access Control: Rejecting connection requests by the SGSN/MME can be implemented without any change to RAN node. MME based access control considers various conditions such as roaming restriction, group etc. in order to determine the rejecting probability which helps to mitigate congestion in core network. Both MME based and RAN based access control mechanisms are discussed in 3GPP specification [3], [11].

3. Admission control method

- Congestion Aware Admission Control Method (CAAC): Authors in [13] [41] proposed and designed an admission control scheme for controlling congestion in M2M networks. It is similar to the former $\mathrm{ACB}$ but the main difference is that in $\mathrm{CAAC}$, the reject probability $p$ is estimated by either MME, or S-GWs/P-GW under congestion condition, whereas ACB has no instruction on how to compute the reject probability $p$. Another difference is that MTC devices are grouped based on their priority classes, 
and the probability $p$ is assigned to each group. Finally, accepting or rejecting MTC traffic is done at the eNBs unlike in ACB where the decision is made at the UEs.

4. Coordination and grouping method:

- K-means and Coordinator Selection Scheme: Coordinate selection from a group of MTC devices becomes a challenging problem. One of the MTC devices acts as a coordinator which works with a particular BS/eNB on behalf of all other MTC devices. In [42] authors proposed some coordinate selection methods with the aim to minimize energy consumption in uplink channels. Most of the MTC devices are battery equipped and have limited lifetime. Energy consumption is one of the most important factors in M2M networks. Figure 3.6 represents the system model of M2M group based networks. Authors derive achievable data rates between BS/eNB and coordinator and between coordinator and M2M device. These data rates are used to calculate total energy consumption (EC) in a MTC system.

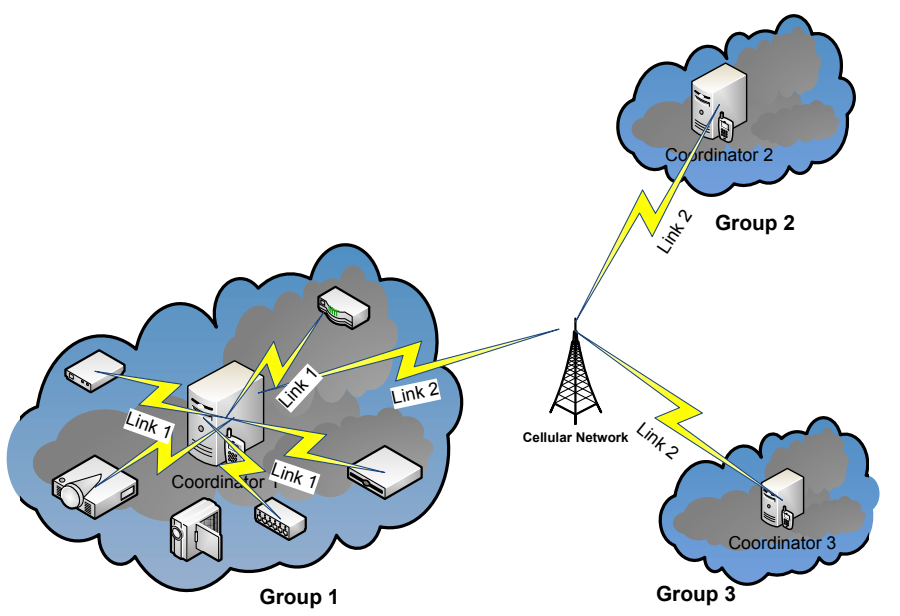

Figure 3.6: Group based M2M communication.

Moreover, authors proposed three different methods to select a coordinator to each group. First method does not consider the channel condition between the coordinator and BS, but chooses coordinator by applying arithmetic means, geometric means of channel gain to other M2M devices. Second method considers channel condition between the coordinator and BS, applying a weighting factor to the channel gain 
information and chooses a M2M device as a coordinator which has maximum channel gain to BS. Last method performs a search to find a minimum EC group along with the coordinator. Simulation results show that the best coordinator is located between the mean point of the group and the nearest point to BS. However, these grouping and coordinate selection mechanisms help to manage massive access to the BS as well as to find a coordinator which helps to minimize energy consumption.

- Cluster-based Access Control: Authors in [39] proposed a group based radio resource management scheme in LTE-A system for QoS guarantees. Group based MTC features are enabled and MTC devices are grouped into different clusters according to their QoS characteristics and packet arrival rate and tolerable jitter value. Group based radio resource management method supports an enormous number of MTC devices.

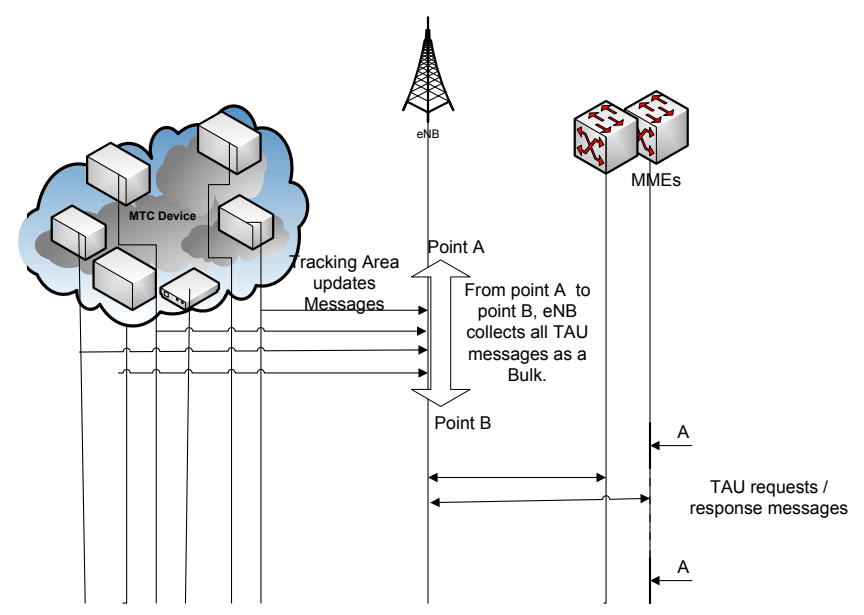

Figure 3.7: Bulk handling in eNB [2].

- Bulk signal handling scheme: This schema is applicable for a group of MTC devices that have a similar signalling message forming as a bulk. In [2], authors showed a potential way to handle bulk messages. Figure 3.7 depicts a scenario to handle a common tracking area update (TAU) message from a group of different MTC devices. It is also effective when non-access stratum (NAS) messages are generated simultaneously by a group of MTC devices. The corresponding eNBs process these NAS signalling in bulk, and forward it towards the MMEs. A similar method proposed in [45], named as 
bundling transmission method in which authors show a multiplexed packet data unit (PDU) format which contains multiple downlink assignment or uplink grant messages for multiple MTC devices. This method reduces overhead in physical control channel when control channel element (CCE) is limited.

- Signalling message compression scheme: Bulk signal handling scheme helps to reduce the amount of traffic but generates extra delay for processing message as a bulk. Authors in [43] propose signalling message compression scheme by creating profile ID. Group of MTC devices and system interface exchange mass amount of messages, for example update location request, cancel location request, tracking area update message etc. These messages contain common information elements (IE) which can be replaced by creating a common profile ID. In [43], authors show the profile ID creation mechanism by reducing message size which helps to reduce processing overhead at the system interfaces and node, respectively.

- Group based packet rates adjustment method: Two major problems will be created by massive access. First one is minimum call rate can not be assured in groups due to RA collision. Second one is wastage of bandwidth. Authors in [44] proposed to change time slots, calculate and define three different packet rate: Offered Packet Rate (OPR), Available Packet Rate (APR) and Minimum Packet Rate (MPR). APR of a group is calculated by summing of all packet rate received by BS. Group OPR is the sum of generated packet rate in all terminals of the group. A fair congestion mechanism is proposed based on these packet rate information.

\subsection{Comparison of different congestion and overload con- trol techniques}

The comparison of different congestion and overload control techniques are shown in Table 3.2. Congestion control techniques that are applied to radio network part mainly focus on the improvement of contention-based random access procedure. Separate radio resources (e.g., time slots, preambles) allocation, different time intervals ( e.g, forbidden time, grant time ) and access class barring methods are proposed to control congestion in RAN. On the other 
side, core network based congestion control techniques attempt to formulate best coordination and grouping methods that help to reduce excessive traffic from the network. M2M grouping based methods save energy, improve throughput as well as mitigate congestion problem in core network.

Table 3.2: Comparison of different congestion and overload control techniques

\begin{tabular}{|c|c|c|c|}
\hline \multicolumn{4}{|c|}{ RAN overload control method } \\
\hline Solution Techniques & Distinguishable features & Performance Metrics & Limitations \\
\hline $\begin{array}{l}\text { Randomize } \\
\text { Access } \\
\text { Method }\end{array}$ & $\begin{array}{l}\text { Refs. [17], [30] use de- } \\
\text { vice's p-persistent value } \\
\text { and backoff timer to de- } \\
\text { lay RA procedure whereas } \\
\text { [32] uses new virtual frame } \\
\text { structure for M2M traffic. }\end{array}$ & $\begin{array}{l}\text {-Minimize access } \\
\text { delay. } \\
\text {-Improve success } \\
\text { rate. }\end{array}$ & $\begin{array}{l}\text { If } n \text { devices initiate RA } \\
\text { in the same time slots, } \\
\text { the eNB can respond to } \\
\text { only one device and the re- } \\
\text { maining } n-1 \text { devices wait } \\
\text { for pre-defined backoff pe- } \\
\text { riod, and then again ini- } \\
\text { tiate RA. This concurrent } \\
\text { procedure increases access } \\
\text { delay. Moreover, this pro- } \\
\text { cedure does not work for } \\
\text { resource constraint M2M } \\
\text { devices. }\end{array}$ \\
\hline $\begin{array}{l}\text { Radio Re- } \\
\text { source } \\
\text { Allocation } \\
\text { Method }\end{array}$ & $\begin{array}{l}\text { The Physical Resource } \\
\text { Blocks (PRBs) are } \\
\text { separated or allocated } \\
\text { dynamically in }[8] \text { [34] } \\
\text { between H2H and M2M } \\
\text { traffic based on the } \\
\text { system load information. }\end{array}$ & $\begin{array}{l}\text {-Ensure the QoS } \\
\text { performance of } \\
\text { H2H traffic. } \\
\text {-Minimize access } \\
\text { delay. }\end{array}$ & $\begin{array}{l}\text { Allocation of } \mathrm{RR} \text { needs } \\
\text { to separate } \mathrm{H} 2 \mathrm{H} \text { traffic } \\
\text { over } \mathrm{M} 2 \mathrm{M} \text { traffic which } \\
\text { imposes a new challenge } \\
\text { to implement this method. }\end{array}$ \\
\hline
\end{tabular}

continued ... 


\begin{tabular}{|c|c|c|c|}
\hline Solution Techniques & Distinguishable features & Performance Metrics & Limitations \\
\hline $\begin{array}{l}\text { Class Bar- } \\
\text { ring Method }\end{array}$ & $\begin{array}{l}\text { Devices are group accord- } \\
\text { ing to their class and } \\
\text { eNB broadcast a param- } \\
\text { eter ( } A C \text { barring factor } \\
\text { ) to reduce the load in } \\
\text { RAN. The key difference } \\
\text { between p-persistent and } \\
\text { ACB [18] mechanism is } \\
\text { that in overload situation, } \\
p \text {-persistent restricts both } \\
\text { H2H and M2M traffic } \\
\text { whereas AC barring factor } \\
\text { uses to restrict M2M traf- } \\
\text { fic. }\end{array}$ & $\begin{array}{l}\text {-Improve through- } \\
\text { put. } \\
\text {-Minimize access } \\
\text { delay. }\end{array}$ & $\begin{array}{l}\text { RAN uses } A C \text { barring fac- } \\
\text { tor to minimize its traffic } \\
\text { load, but there is no clear } \\
\text { mechanism yet proposed } \\
\text { to define this value. More- } \\
\text { over, these solution meth- } \\
\text { ods consider same bearing } \\
\text { factor and barring time for } \\
\text { all category MTC devices. }\end{array}$ \\
\hline $\begin{array}{l}\text { eNB Selec- } \\
\text { tion Method }\end{array}$ & $\begin{array}{l}\text { Ref. [37] considers relative } \\
\text { signal strength, available } \\
\text { channel gain and packet } \\
\text { transmission time as a pa- } \\
\text { rameter to select eNB. }\end{array}$ & $\begin{array}{l}\text {-Minimize access } \\
\text { delay } \\
\text {-Improve QoS }\end{array}$ & $\begin{array}{l}\text { This method only consid- } \\
\text { ers those devices which re- } \\
\text { side into ovelapping area } \\
\text { of multiple eNBs. }\end{array}$ \\
\hline \multicolumn{4}{|c|}{ CN overload control method } \\
\hline $\begin{array}{l}\text { Access Class } \\
\text { Barring } \\
\text { Method }\end{array}$ & $\begin{array}{l}\text { Enhanced version of ACB } \\
{[19][18][24] \text { are used to }} \\
\text { balance the load between } \\
\text { core network and radio } \\
\text { network. In [18], ACB pa- } \\
\text { rameter is updated based } \\
\text { on the load information } \\
\text { whereas in [24], all BS } \\
\text { jointly decide this param- } \\
\text { eter. }\end{array}$ & $\begin{array}{lr}\quad[24] & \text { improves } \\
\text { system } & \text { throughput } \\
\text { and } & \text { minimizes } \\
\text { access } & \text { delay } \\
\text { while } & {[18] \quad[19]} \\
\text { reduces } & \text { signalling } \\
\text { overhead } & \text { and im- } \\
\text { proves } & \text { success } \\
\text { probability. }\end{array}$ & $\begin{array}{l}\text { Integration of different } \\
\text { class dependent barring } \\
\text { factor and barring time } \\
\text { with EAB is consid- } \\
\text { ered as a new scope of } \\
\text { improvement. }\end{array}$ \\
\hline
\end{tabular}

continued ... 


\begin{tabular}{|l|l|l|l|}
\hline Solution Techniques & Distinguishable features & Performance Metrics \\
Control & $\begin{array}{l}\text { Estimate reject probability } \\
\text { Method }\end{array}$ & $\begin{array}{l}\text {-Reduces signalling } \\
\text { and reject factor }(R F) \text { to } \\
\text { overhead. } \\
\text { fic. }\end{array}$ & $\begin{array}{l}\text { The QoS performance } \\
\text { of H2H traffic become } \\
\text { affected by this method, } \\
\text { because this method uses } \\
\text { reference queue length } \\
\text { to estimate reject factor, } \\
\text { which rejects both M2M } \\
\text { and H2H traffic. }\end{array}$ \\
\hline $\begin{array}{l}\text { Coordination } \\
\text { ing Method }\end{array}$ & $\begin{array}{l}\text { In } \\
\text { are clustered according to } \\
\text { their different QoS char- } \\
\text { acteristics (e.g. packet } \\
\text { arrival rate, jitter value } \\
\text { etc.). } \\
\text { tures of other group based } \\
\text { method }[2][45] \text { and [43] } \\
\text { are data aggregation and } \\
\text { compression of signalling } \\
\text { messages. }\end{array}$ & $\begin{array}{l}\text {-Improves QoS per- } \\
\text { formance. } \\
\text {-Reduces signalling } \\
\text { overhead. }\end{array}$ & $\begin{array}{l}\text { Energy efficient group for- } \\
\text { mation and the best co- } \\
\text { ordinator or group leader } \\
\text { selection among MTC de- } \\
\text { vices are the new possibil- } \\
\text { ities to improve grouping } \\
\text { methods. }\end{array}$ \\
\hline
\end{tabular}

In the next chapter, we will discuss an analytical solution for massive access management for MTC traffic and propose a dynamic access class barring method which distribute MTC traffic among base stations using load balancing method. 


\section{Chapter 4}

\section{Proposed Dynamic Access Class Barring Method}

In this chapter, firstly, we analyze the impact of slotted Aloha random access procedure for M2M traffic in terms of throughput, access success probability and access collision probability. Then, we propose our analytical solution for controlling massive access requests for M2M traffic. We devise a base station selection and load balancing method. Finally, we propose a dynamic access class barring method to balance massive access requests among base station over the cellular M2M networks.

\subsection{Preliminary analysis of RA in M2M traffic}

System performance of cellular networks such as throughput, access success probability, transmission delay are greatly influenced by multiple access protocols. In cellular networks, both M2M and $\mathrm{H} 2 \mathrm{H}$ users initiate contention-based random access procedure to setup radio resource control (RRC) connection to the network. Basically, contention-based slotted Aloha random access procedure is suitable for larger cell sized networks. Therefore, M2M traffic uses this method to send contention requests. In this section, we describe the impact of slotted Aloha random access (RA) procedure when it accommodates both MTC and H2H traffic.

Let random access requests arrive in Poisson arrival with arrival rate $\frac{\gamma}{N}$ where $\gamma=M \lambda$. Here, $M$ represents the total number of users, $\lambda$ denotes packet arrival rate and $N$ denotes random 
access opportunity (RAO). In LTE, RAOs define random access channel (RACH) capacity. There are $64 \mathrm{RACH}$ configuration indexes available and each index allocates different slot for RA. Figure 4.1 shows RA slots when RACH configure index is set to 6 . In each slot, each user

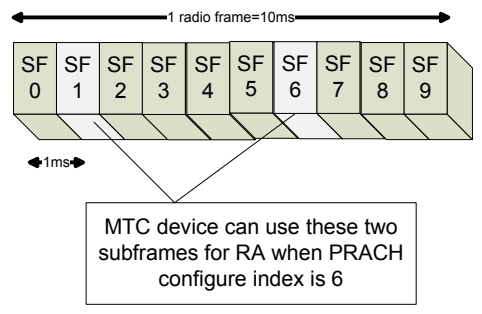

Figure 4.1: RA slots when RACH configure index is 6 .

chooses one preamble sequence out of 64 and sends it through the dedicated RA slot. With the Poisson arrival process, the throughput of per RAO is calculated as

$$
\tau=\frac{\gamma}{N} e^{-\frac{\gamma}{N}}
$$

In the slotted Aloha system, the total throughput of all opportunity can be defined as

$$
T=N \times \tau=\gamma e^{-\frac{\gamma}{N}}
$$

where $\tau$ denotes throughput per RAO. We analyze slotted Aloha RA by increasing system loads (e.g. number of users). In the first case, we investigate the performance of slotted Aloha RA considering $20 \mathrm{H} 2 \mathrm{H}$ users and different number of M2M users and, 200 RAOs by changing the packet arrival rates (e.g. 0.4 packets/sec to 1.6 packets/sec). In the second case, we consider arrival rate $=0.2$ packets $/ \mathrm{sec}$ and apply different RAOs.

It can be observed from Figure 4.2 that the system throughput depends on three factors: the number of users, packet arrival rate and the number of access opportunity.

Figure 4.3(a) shows that success probability of RA increase with the increase in random access opportunity. The more opportunity means more users can send RA contention requests. Figure 4.3(b) also shows the collision probability in term of random access opportunity. Figures 4.2 and 4.3 show that the total random access opportunity, packets arrival rate and the number of users control the success rate and throughput of the S-Aloha system. In the next section, we propose an analytical model by considering these performance factors of S-Aloha for M2M enabled cellular networks. 

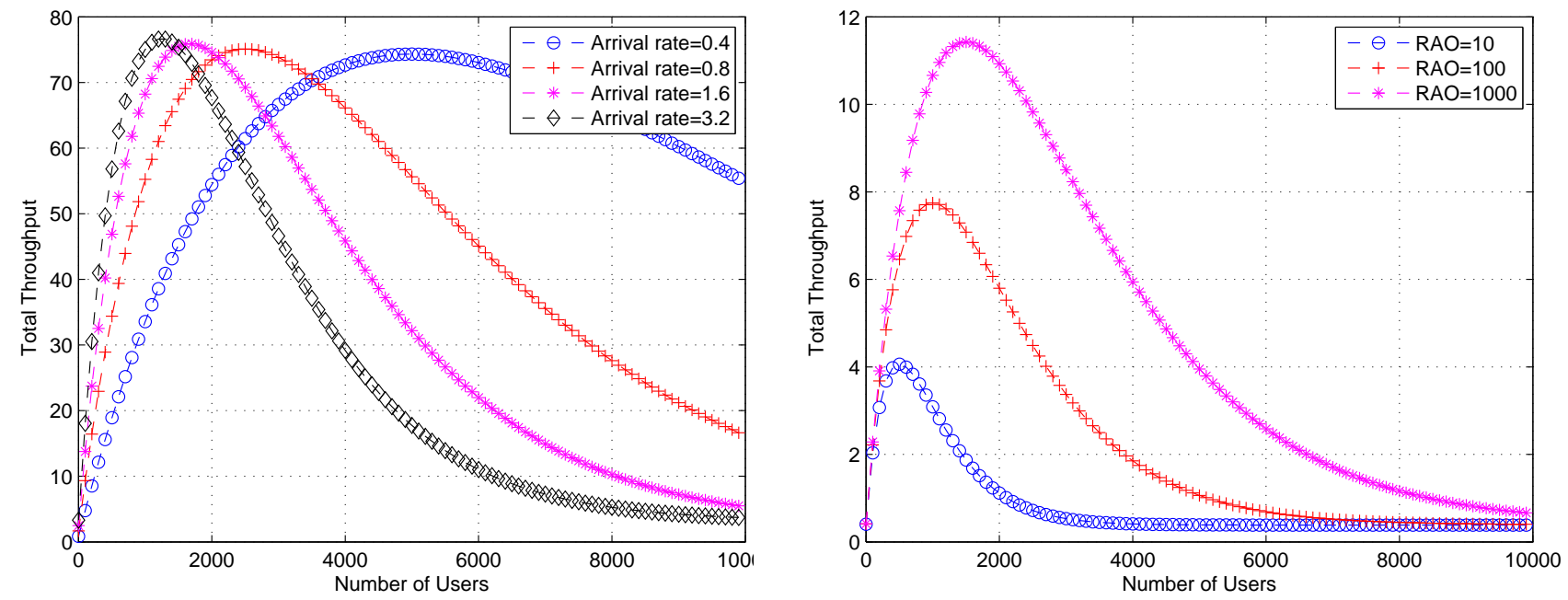

(a) Total throughput with different arrival rate.

(b) Total throughput with different random access opportunity.

Figure 4.2: Performance analysis (total throughput) of slotted Aloha random access vs number of system load (both $\mathrm{H} 2 \mathrm{H}$ and M2M users).
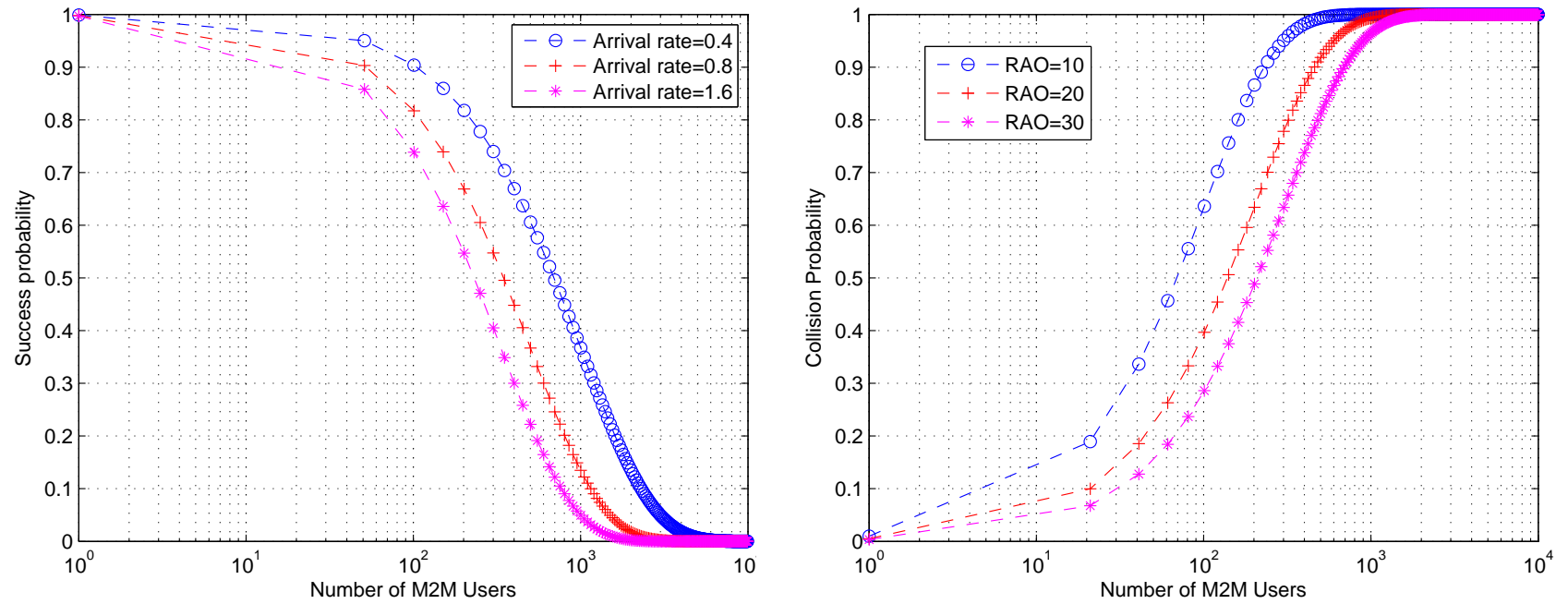

(a) Success probability of slotted Aloha random ac- (b) Collision probability of slotted Aloha random cess vs number of M2M users. access vs number of M2M users.

Figure 4.3: Performance analysis (success probability and collision probability) of slotted Aloha random access vs number of system load (both H2H and M2M users). 


\subsection{Analytical model}

\subsubsection{System model and problem formulation}

In a LTE network, each user sends RA request to its neighbor base station (e.g. eNB) in order to synchronize with the network. The eNB validates this request and if successful, it grants some uplink channel to the user. The M2M users employ the similar procedure. M2M devices are deployed all over the network as shown in Figure 4.4. We consider those devices which can send RA requests to more than one base station. Figure 4.4 depicts a network model in which overlapping areas are the main focus of this work to develop an analytical model.

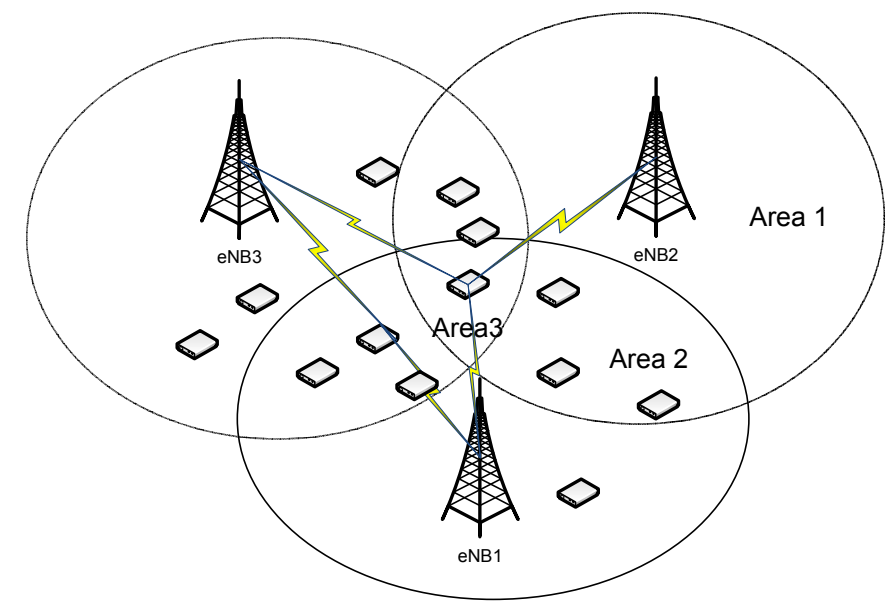

Figure 4.4: System model

In Figure 4.4, devices in area 2 and area 3 can send requests to more than one eNB. When a large number of M2M devices initiate RA procedure, the appropriate eNB selection method can help to reduce congestion probability, access delay as well as improve throughput.

Let there be $M$ devices covered by $\psi$ base stations in the overlapping area. The objective function of selecting eNBs is formulated as:

$$
T=\arg \max _{\forall D_{i}}\left(T_{e N B_{j}}\right) \text { where } i=1 . . M \text { and } j=1 \ldots \phi,
$$

where $T$ denotes the total throughput of the system and $T_{e N B_{j}}$ represents the throughput of particular base station $j$ after serving $i$ number of devices. $D_{i}$ represents $i$ th device and $\mathbf{D}$ denotes the vector of devices. 
This objective can be satisfied by applying maximum asposterior probability (MAP) criterion between devices and base stations and estimate MAP of each base station $P\left(e N B_{j} \mid \mathbf{D}\right) \forall_{j}$. In the next section, we discuss the derivation of this probability.

\subsubsection{Proposed solution}

\subsubsection{Base station selection method}

Figure 4.5(a) shows the scenario where we apply base station selection mechanism and Figure 4.5(b) shows the corresponding probabilistic model. To evaluate the performance of RACH procedure, $3 \mathrm{GPP}$ defines various performance metrics such as success probability, collision probability, access delay, packet transmission delay [7]. Authors in [46] present an analytical model to show the inconsistency of the definition of success and collision probability for the perspective of RAO and overload control.

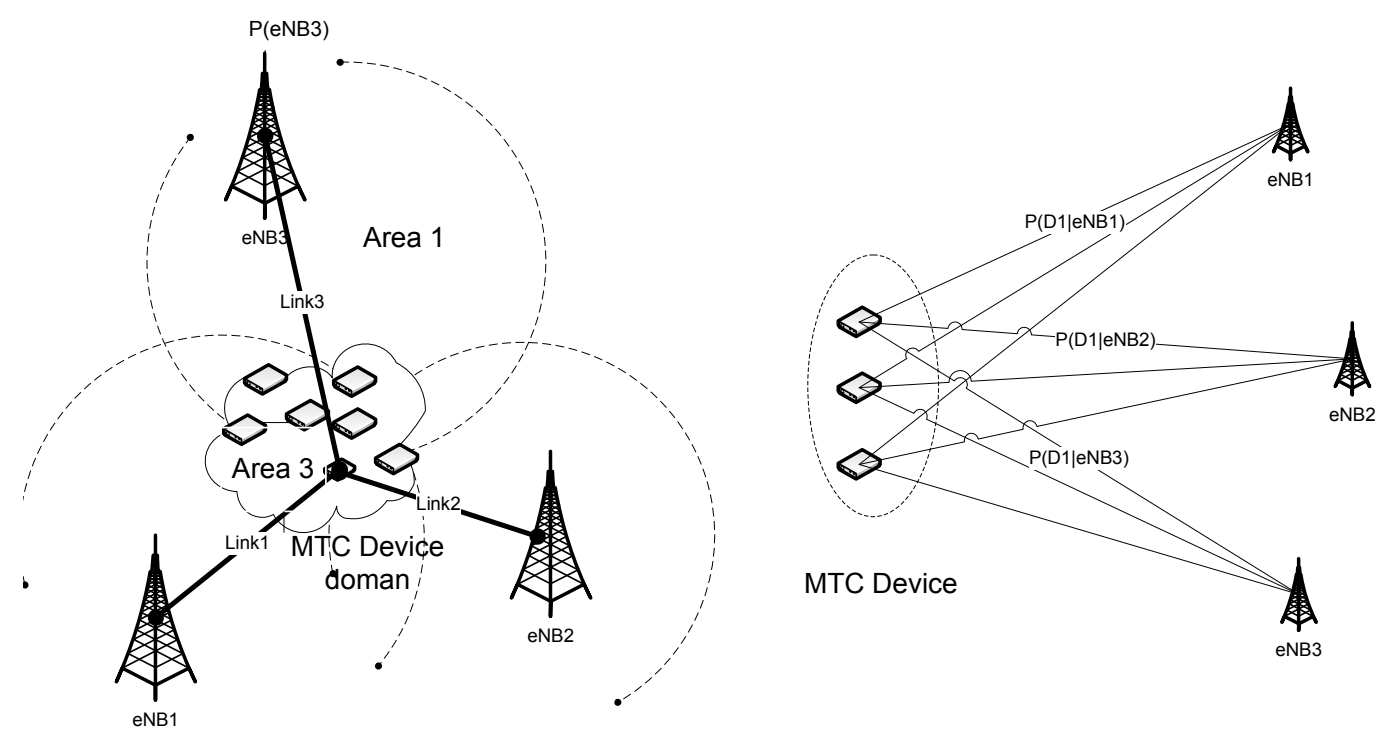

(a)

(b)

Figure 4.5: Probabilistic Model

In [7], the success probability of preamble transmission of a device is defined as $P_{s}(\mathbf{D})=e^{-\frac{\gamma}{N}}$. We can consider this probability as $P(\mathbf{D} \mid e N B)$. This means the probability of $\mathbf{D}$ given a particular base station (eNB). Again, here, D represents the vector of M2M devices. 


$$
P(\mathbf{D} \mid e N B)=P_{s}(\mathbf{D})=e^{-\frac{\gamma}{N}}
$$

In Figure 4.5, let area 3 cover $M$ number of devices and each device sends a request message with an arrival rate $\lambda$. The total arrival rate in this area will be $\gamma=M \lambda$. $N$ represents the random access opportunity (RAO) which is calculated by the number of RA slots and the number of preamble sequences. For example, if RACH configure index is set to 6 , then per radio frame has $R A O=2 \times 54=108$. If the base station responds $r$ frames per second, then the outgoing traffic will be $r$ frames/sec. In this case, RAOs are increased by the factor of $r$ that is $R A O=2 \times 54 \times r$. Then the conditional probability will be

$$
P(\mathbf{D} \mid e N B)=e^{-\frac{\gamma}{N r}}
$$

Again, the devices in area 3 can choose any surrounding base station. The prior probability of all base stations will be the same. If there are $\phi$ base stations covering this area, the prior probability will be

$$
P\left(e N B_{1}\right)=P\left(e N B_{2}\right)=\ldots=P\left(e N B_{\phi}\right)=\frac{1}{\phi} .
$$

The objective function of selecting an appropriate eNB is to increase the throughput of the base station. To achieve this objective, the eNB selection probability will be maximum asposterior probability which can be estimated as:

$$
P\left(e N B_{i} \mid \mathbf{D}\right)=\frac{P\left(\mathbf{D} \mid e N B_{i}\right) P\left(e N B_{i}\right)}{p(x)},
$$

where $p(x)$ will be

$$
p(x)=\sum_{i=1}^{\phi} \sum_{j=1}^{M} P\left(D_{j} \mid e N B_{i}\right) P\left(e N B_{i}\right) .
$$

\subsubsection{Load Balancing}

In the access class barring (ACB) [18] [19] schema, each base station sets a parameter called AC barring factor $(\alpha)$ and broadcasts this value through the system information block 2 (SIB 2). In the high load situation, each base station sets a low value which restricts the users to access the network. The details of ACB and its different derivative such as enhanced ACB, cooperative 


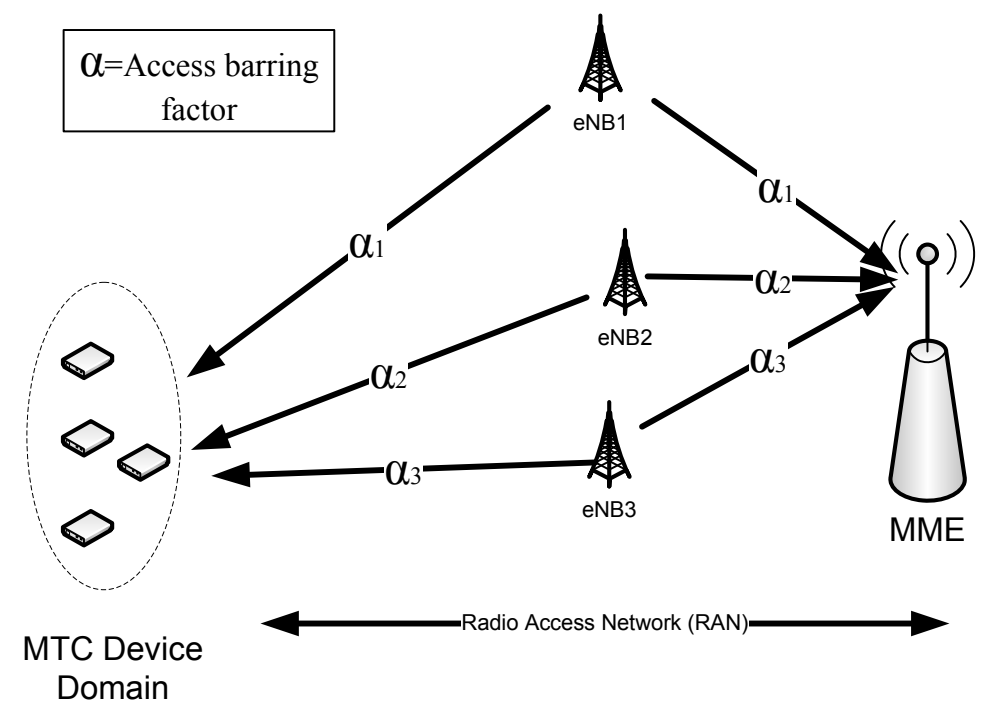

Figure 4.6: System model for load balancing.

ACB are described in Chapter 3. A dynamic ACB scheme is proposed which balances the load among the base stations through $\alpha$. Figure 4.6 is considered as a system model for load balancing. Every base station connects to the core network through the mobility management unit (MME). The MME knows the status of each base station and also the broadcasted parameter $\alpha$ that are sent by each base station. The MME can control the load among base stations through the AC barring parameter. One method is that MME can simply average this value and send it to all the connected base stations. Another method is that each base station sets its success probability as their $\alpha$ parameter and broadcasts it. That is,

$$
\alpha_{1}=P\left(\mathbf{D} \mid e N B_{1}\right), \alpha_{2}=P\left(\mathbf{D} \mid e N B_{2}\right) \ldots . . \alpha_{\phi}=P\left(\mathbf{D} \mid e N B_{\phi}\right) .
$$

When MME gets these values, it does the averaging and sends it back to the base station.

$$
\alpha^{*}=\frac{\alpha_{i}}{\sum_{i=1}^{\phi} \alpha_{i}}=\frac{P\left(\mathbf{D} \mid e N B_{i}\right)}{\sum_{i=1}^{\phi}\left(P\left(\mathbf{D} \mid e N B_{i}\right)\right)} .
$$

The MME periodically checks the status of all connected base stations and adjusts AC barring parameter. 


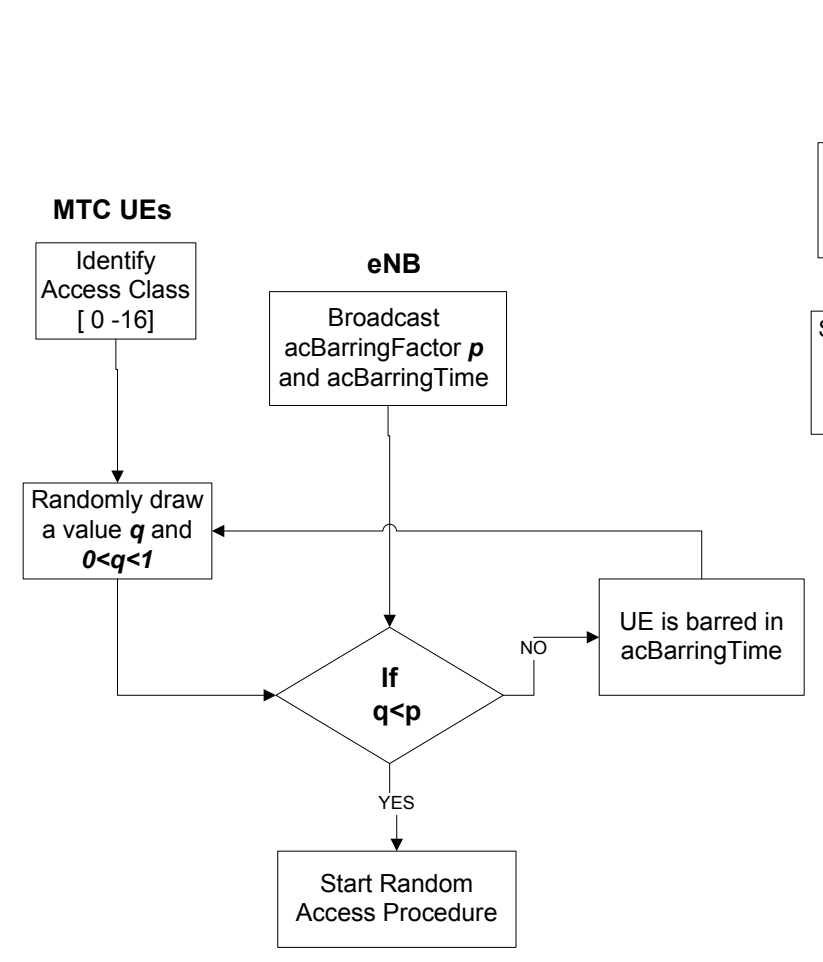

(a) ACB method.

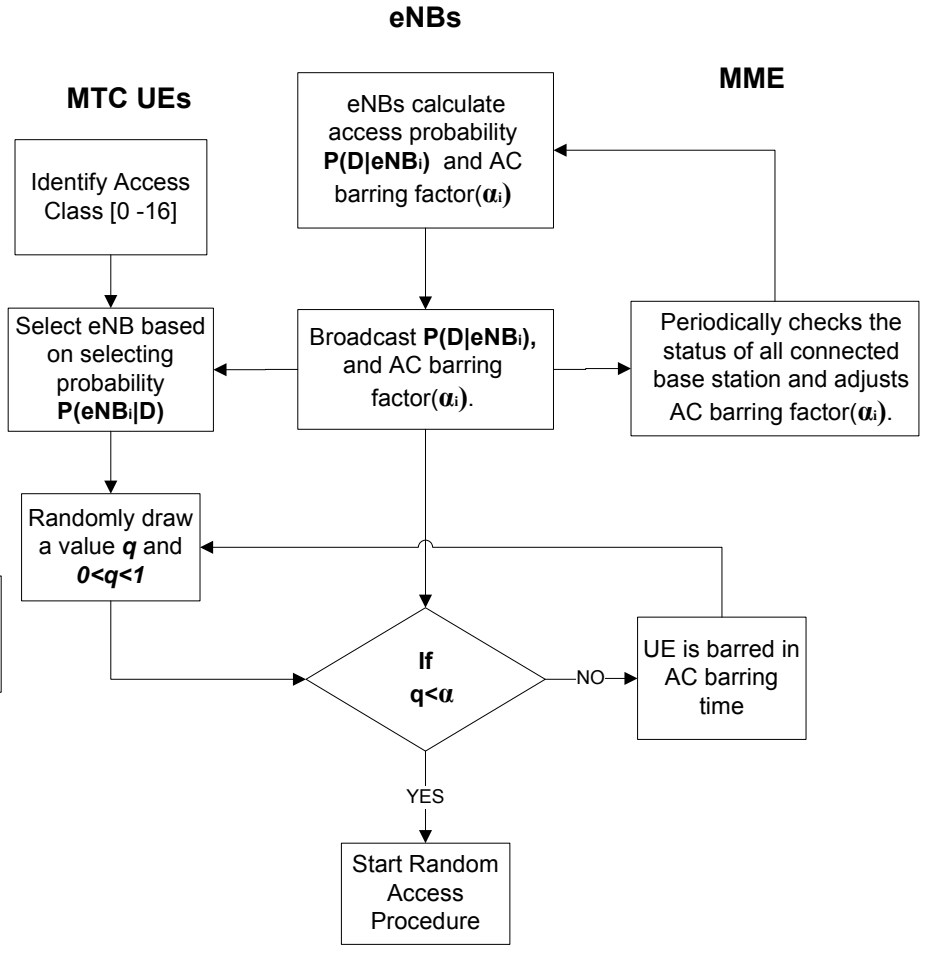

(b) Dynamic ACB method.

Figure 4.7: Flow chart of general ACB and proposed dynamic ACB method.

\subsection{Proposed dynamic access class barring method}

Our proposed dynamic access class barring method integrates both base station selection and load balancing method with ACB method. Figure 4.7(b) shows the flow diagram of dynamic ACB method. This method works as follows:

- Step 1: Each eNB $i$ estimates access probability $P\left(\mathbf{D} \mid e N B_{i}\right)$ based on their packet arrival rate and transmission rate, and access class barring parameter $\left(\alpha_{i}\right)$ based on the physical random access channel (PRACH) condition.

- Step 2: Every eNB periodically broadcasts access probability and AC barring parameter.

- Step 3: The MME periodically checks the status of all connected base stations and adjusts AC barring parameter.

- Step 4: Each MTC device or group of MTC device selects a particular eNB based on the 
maximum asposterior probability (MAP) $P\left(e N B_{i} \mid \mathbf{D}\right)$.

- Step 5: Each MTC device generates a random number $q, 1>q>0$ and performs normal ACB mechanism for random access.

Figure 4.7(a) shows the flow diagram of the general ACB method. The key difference of ACB and dynamic ACB method are:

1. In dynamic ACB, each device can choose base station based on MAP. In ACB, no such mechanism is present.

2. A dynamic $\mathrm{AC}$ barring parameter is adjusted periodically according to the load condition among base station. In ACB method, there has no clear mechanism yet been described in the literature for how to determine AC barring parameter.

\subsection{Simulation parameters}

The proposed dynamic ACB scheme is evaluated through simulation. In the simulation, we consider two base stations, $20 \mathrm{H} 2 \mathrm{H}$ users and maximum 10,000 M2M users. Table 4.1 shows the other simulation parameters.

Table 4.1: Simulation Parameters.

\begin{tabular}{|c|c|}
\hline Parameters & Values \\
\hline System bandwidth $(W)$ & $5 \mathrm{MHz}$ \\
\hline RACHConfigureIndex & 6 \\
\hline Arrival process & Poisson \\
\hline Arrival rate $(\lambda)$ & 0.2 \\
\hline Frame transmission rate $(F T R)$ & $10,60,100,200$ \\
\hline ACBarring factor $(\alpha)$ & $0.5,0.8$ \\
\hline
\end{tabular}




\subsection{Simulation results}

Two cases are considered for analysis of the results.

\subsubsection{Impact of frame transmission rate}

In this case, we investigate the impact of throughput and random access success probability of two base stations (eNBs) by applying different frame transmission rates $(F T R)$ and access class barring parameter $(\alpha)$.

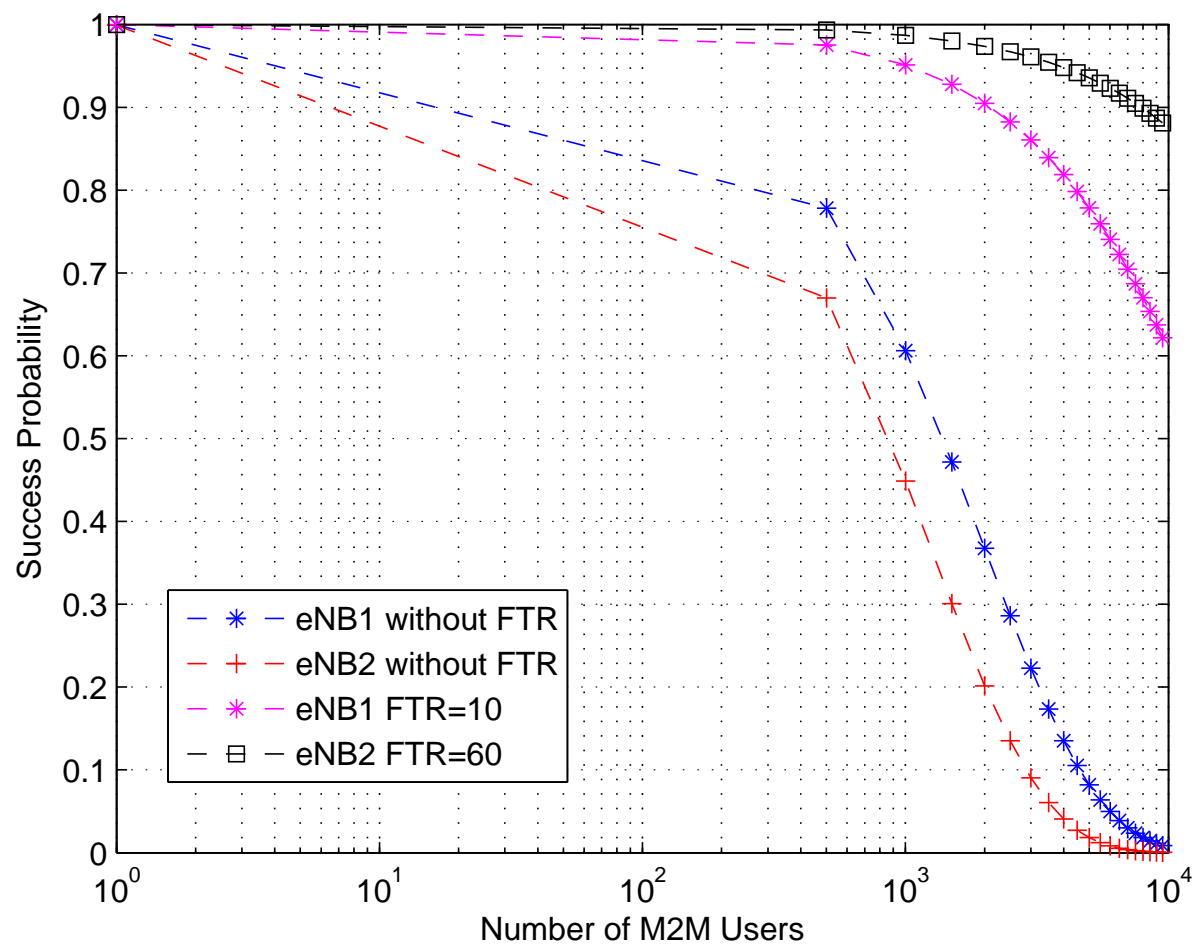

Figure 4.8: Success probability of eNBs considering $\alpha_{1}=0.5, \alpha_{2}=0.8$ and $F T R_{1}=10$ and $F T R_{2}=60$.

Figure 4.8 shows that RA success probability and throughput of both base stations improve significantly when frame transmission rate is considered in random access opportunity. By increasing RAOs, both base stations serve a large number of RA requests. 


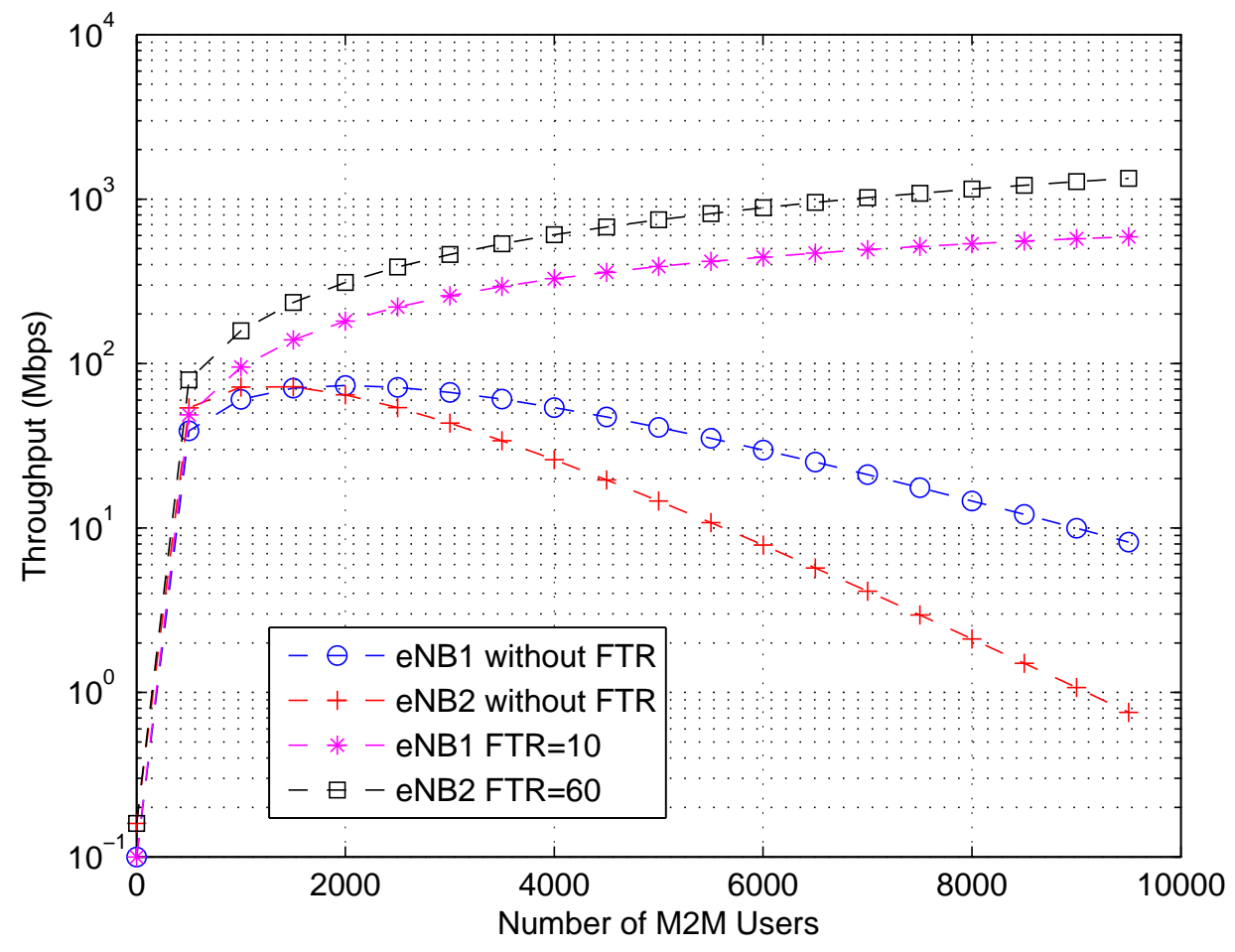

Figure 4.9: Throughput estimation of eNBs considering $\alpha_{1}=0.5, \alpha_{2}=0.8$ and $F T R_{1}=10$ and $F T R_{2}=60$.

In Figure 4.9, the overall throughput improvement rate depends on transmission rate and $\mathrm{ACB}$ parameter. In this case, eNB2 shows better performance as its transmission rate is higher than that of the other base station.

\subsubsection{Impact of dynamic ACB schema}

We investigate the performance of dynamic $\mathrm{ACB}$ with different frame transmission rate and ACB parameter. Nine cases are considered in this simulation. In each case, firstly, we apply load balancing mechanism between two base stations and then apply eNB selection method. We categorize these nine cases into three groups depending on the frame transmission rate. The groups are:

- Group one: both base stations have same frame transmission rate $\left(F T R_{1}=F T R_{2}\right)$.

- Group two: both base stations have different frame transmission rate $\left(F T R_{1}>F T R_{2}\right)$.

- Group three: base station two has higher frame transmission rate than one $\left(F T R_{1}<F T R_{2}\right)$. 
Simulation results of these groups are presented in the next sub-section.

\subsubsection{Group one: $F T R_{1}=F T R_{2}$}

Figure 4.10 shows the simulation results of group one. In this group, the arrival rate of RA request is set to 0.2 and transmission rates of both stations are considered as same. Here, we consider three different cases by assuming different initial ACB parameter for both base stations. The cases are:

- Case 1(a): Both base stations set same ACB parameter for managing the massive access requests. After applying dynamic ACB method, both base stations show the same performance which is depicted in the Figures 4.10(a). In this case, the selection probability of both base stations (e.g. in 4.10(b)) shows equal probability.

- Case 1(b): Initially, the ACB parameter of base station one is higher than that of base station two (e.g. $\alpha_{1}>\alpha_{2}$ and assuming $\alpha_{1}=0.8, \alpha_{2}=0.5$ ), as shown in Figures 4.10(c) and 4.10(d). In this case, initially $e N B_{2}$ provides better throughput than one because lower value of the ACB parameter (e.g. $\left.\alpha_{2}\right)$ restricts higher number of contention requests. The throughput of $e N B_{1}$ drops significantly after 1500 random access requests whereas $e N B_{2}$ 's throughput slightly decreases after 2000 requests. An interesting fact has been observed from the Figure 4.10(c), that is, after applying dynamic ACB parameter along with equal frame transmission rates, both base stations support a large number of $\mathrm{M} 2 \mathrm{M}$ device requests compared to the fixed allocation ACB parameters. The dynamic ACB increases throughput of the both base stations. Both base stations show equal performance after 2000 RA requests, because the dynamic ACB method balances the $\alpha_{1}$ and $\alpha_{2}$ parameters after receiving $2000 \mathrm{RA}$ requests.

- Case 1(c): In this case, the base station two sets higher ACB parameter than one, (e.g. $\alpha_{1}<\alpha_{2}$ and assuming $\alpha_{1}=0.5$ and $\left.\alpha_{2}=0.8\right)$, as shown in Figures 4.10(e) and 4.10(f). As $\alpha_{1}<\alpha_{2}$, initially two base station show opposite results than case 1(b). However, after applying dynamic ACB method with equal FTR, both base stations show similar results as case 1(b). 


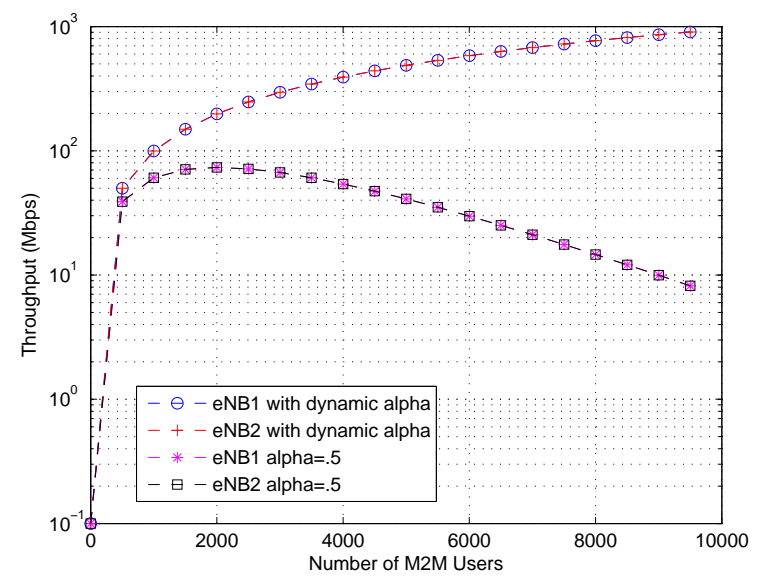

(a) Throughput estimations considering $\alpha_{1}=\alpha_{2}$.

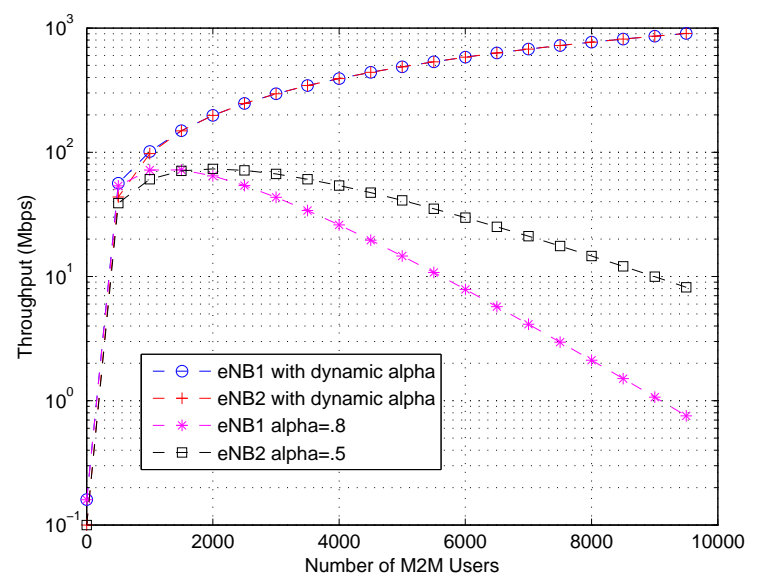

(c) Throughput estimations considering $\alpha_{1}>\alpha_{2}$.

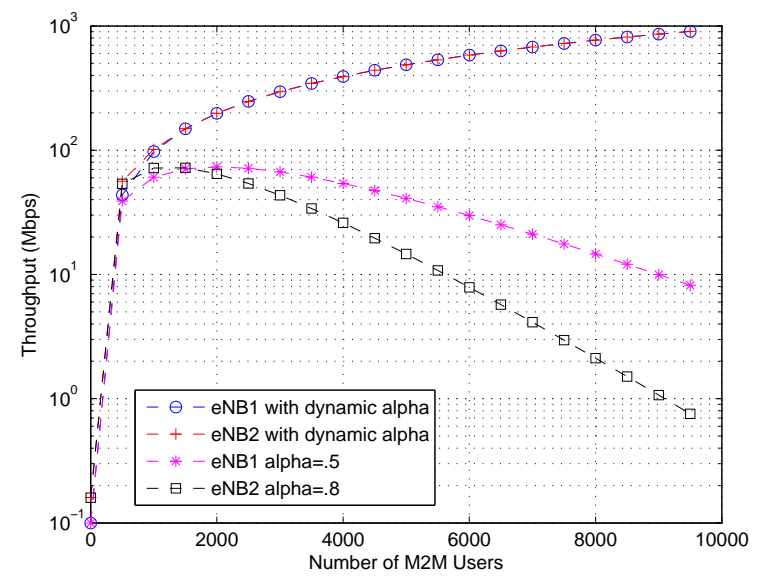

(e) Throughput estimations considering $\alpha_{1}<\alpha_{2}$.

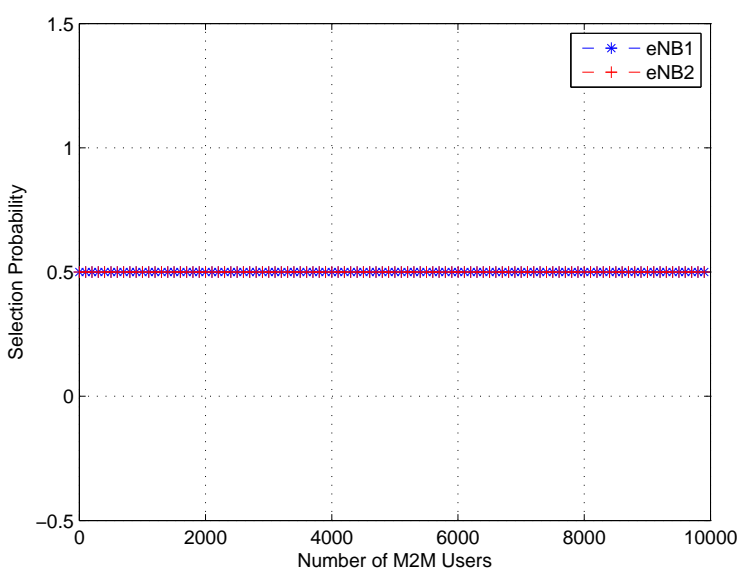

(b) Selection probability (initially $\alpha_{1}=\alpha_{2}$ ).

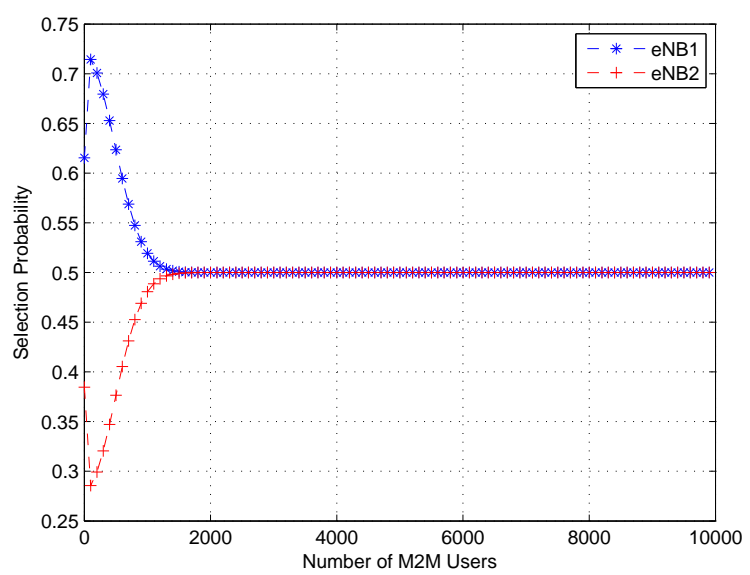

(d) Selection probability (initially $\alpha_{1}>\alpha_{2}$ ).

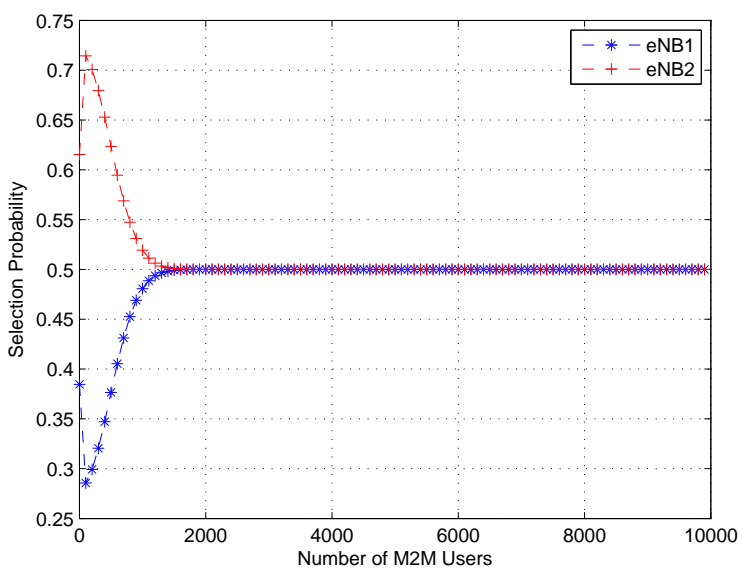

(f) Selection probability (initially $\alpha_{1}<\alpha_{2}$ ).

Figure 4.10: Throughput and selection probability of eNBs when applying dynamic ACB with $F T R_{1}=F T R_{2}$. 
In general, the Figures 4.10(a), 4.10(c) and 4.10(e) show the throughput of the base station before and after applying dynamic ACB method. It has been observed from these figures that after applying dynamic ACB method, both base stations give the equal performance after receiving around 2000 random access requests. The eNB selection probability is shown in Figures 4.10(b), 4.10(d) and 4.10(f). As both stations have equal transmission rates but different ACB parameter, the proposed dynamic ACB adjusts the barring parameter and balances the load between two stations. In this case, after a particular time instant, devices can choose any base station to send their contention requests.

\subsubsection{Group two: $F T R_{1}>F T R_{2}$}

Likewise group one, we consider similar cases in group two but with different transmission rate. The cases are:

-Case 2(a): Both base stations set the same ACB parameter, as shown in Figures 4.11(a) and 4.11(b). Before applying dynamic ACB method, the base stations set equal ACB parameters without FTR, and they show equal performance in term of throughput and the throughput drop around receiving 1600 RA requests. After applying $\mathrm{ACB}$ method with $F T R_{1}>F T R_{2}$, both base stations increase the throughput and support a large number of access requests compared to the fixed allocation ACB method.

-Case 2(b): The ACB parameter of base station one is higher than the base station two (e.g. $\alpha_{1}>\alpha_{2}$ ). It can be observed from Figure 4.11(c) that $e N B_{1}$ shows significantly improvement in throughput when applying highest frame transmission rate and dynamic ACB method.

-Case 2(c): The Figures 4.11(e) and 4.11(f) show the results of this case. In this case, the base station two sets higher ACB parameter than one, (e.g. $\alpha_{1}<\alpha_{2}$ ).

Figures 4.11(a), 4.11(c) and 4.11(e) show the results for load balancing method. As the $e N B_{1}$ has higher transmission rate, it shows better performance than $e N B_{2}$. This can be justified by the fact that the higher transmission rate gives more random access opportunity to $e N B_{1}$. Moreover, dynamic ACB parameter adjusts the load between two base stations. It can be observed in Figures 4.11(c) and 4.11(e) that the overall throughput of the base station two also increase significantly after applying dynamic ACB method. 


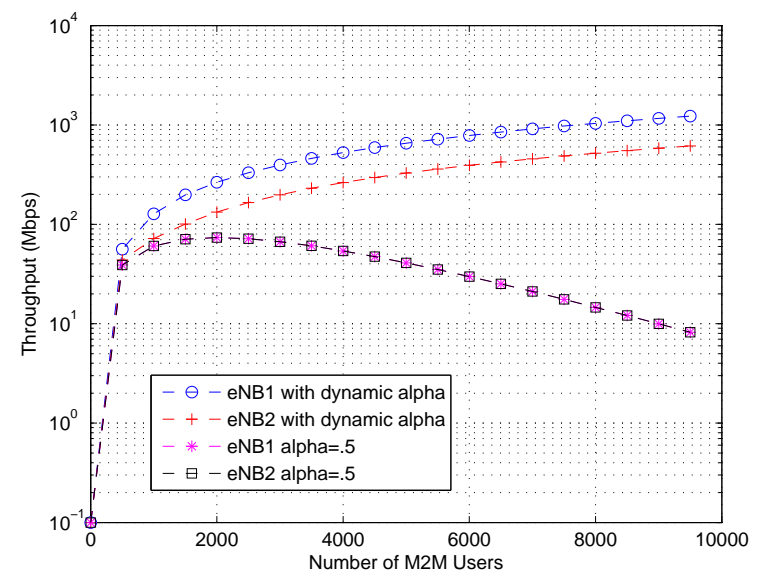

(a) Throughput estimations considering $\alpha_{1}=\alpha_{2}$.

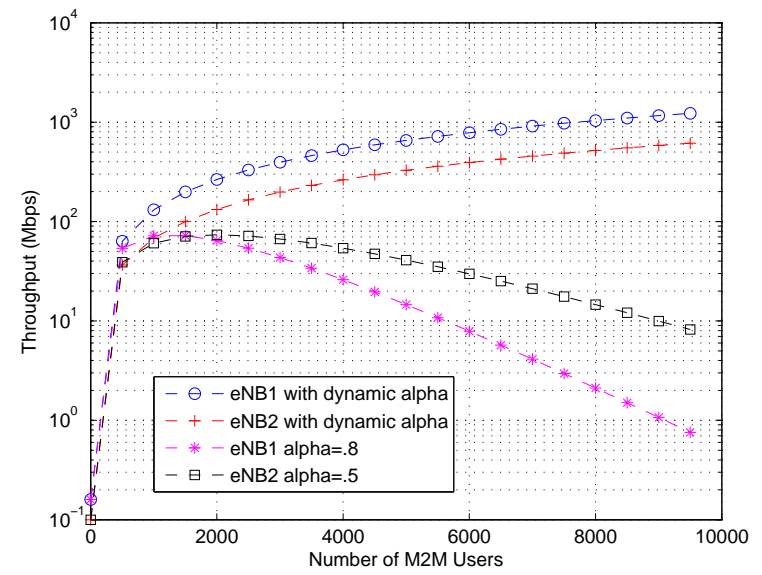

(c) Throughput estimations considering $\alpha_{1}>\alpha_{2}$.

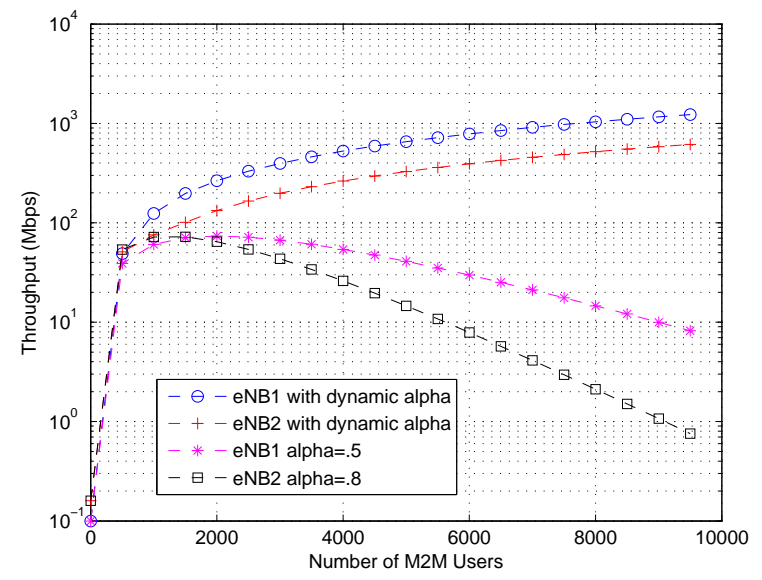

(e) Throughput estimations considering $\alpha_{1}<\alpha_{2}$.

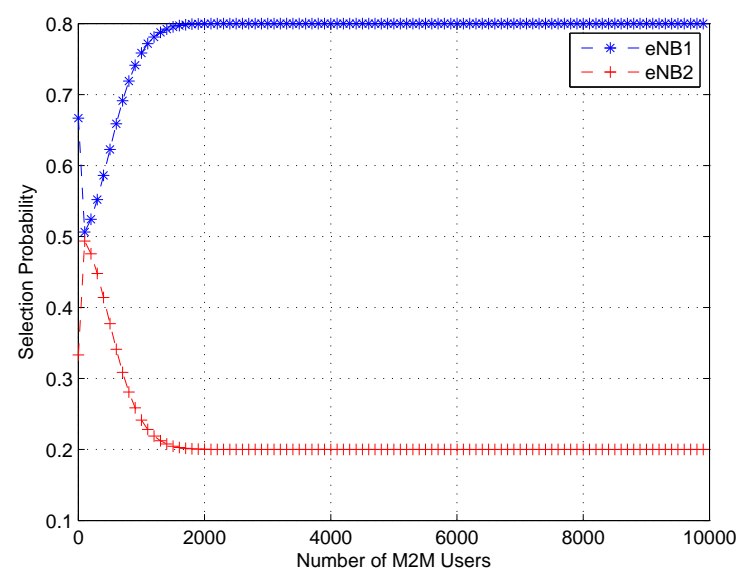

(b) Selection probability (initially $\alpha_{1}=\alpha_{2}$ ).

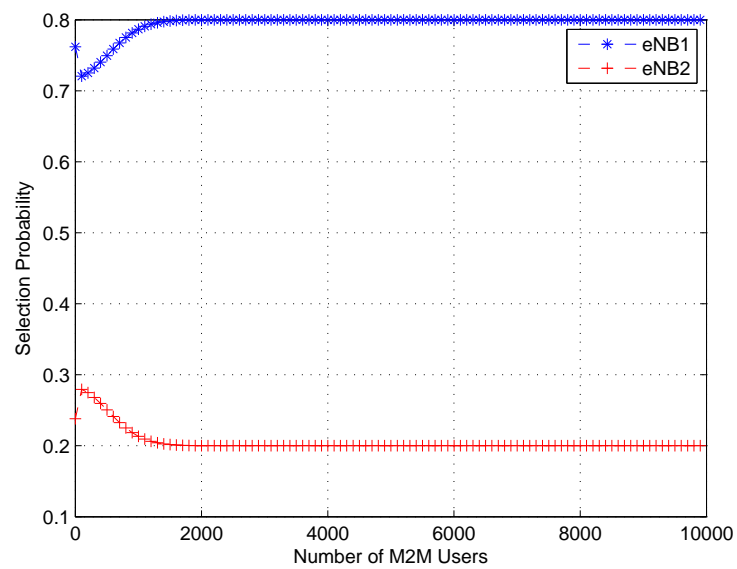

(d) Selection probability (initially $\alpha_{1}>\alpha_{2}$ ).

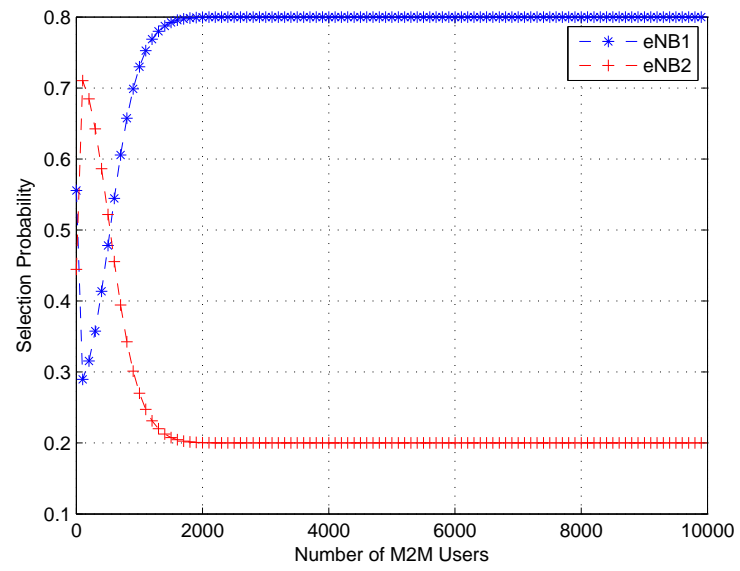

(f) Selection probability (initially $\alpha_{1}<\alpha_{2}$ ).

Figure 4.11: Throughput and selection probability of eNBs when applying dynamic ACB with $F T R_{1}>F T R_{2}$. 


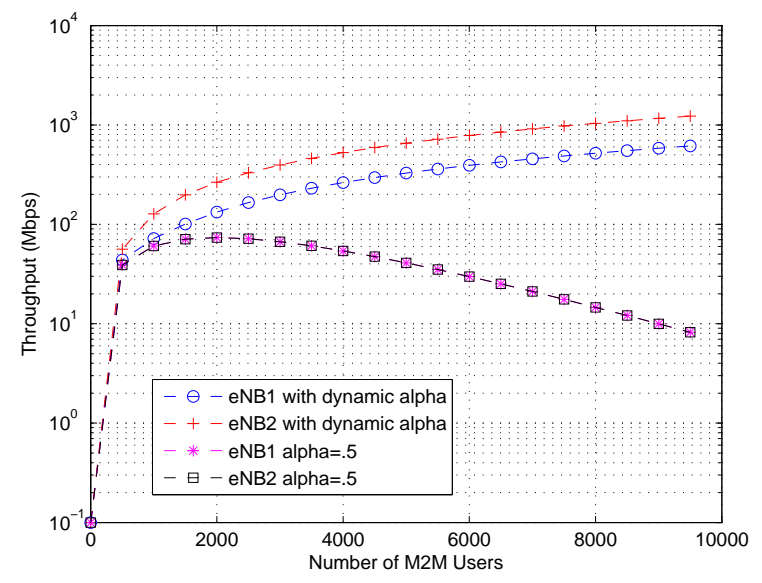

(a) Throughput estimations considering $\alpha_{1}=\alpha_{2}$.

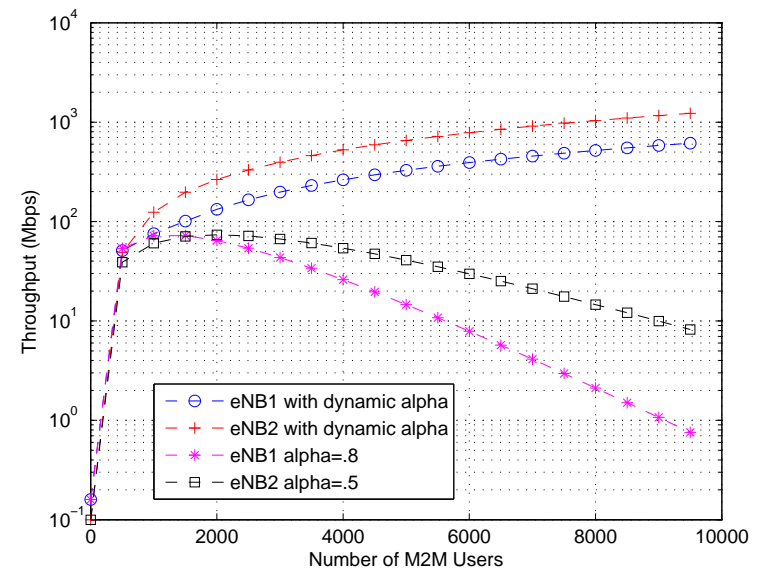

(c) Throughput estimations considering $\alpha_{1}>\alpha_{2}$.

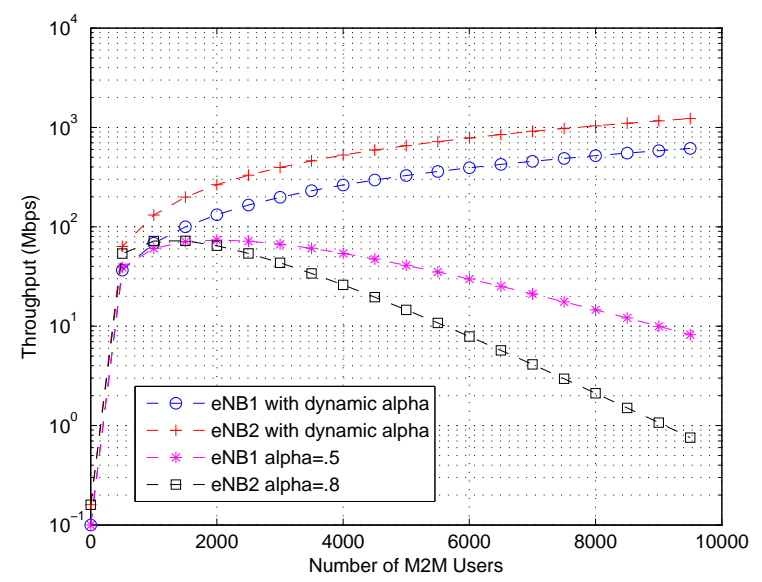

(e) Throughput estimations considering $\alpha_{1}<\alpha_{2}$.

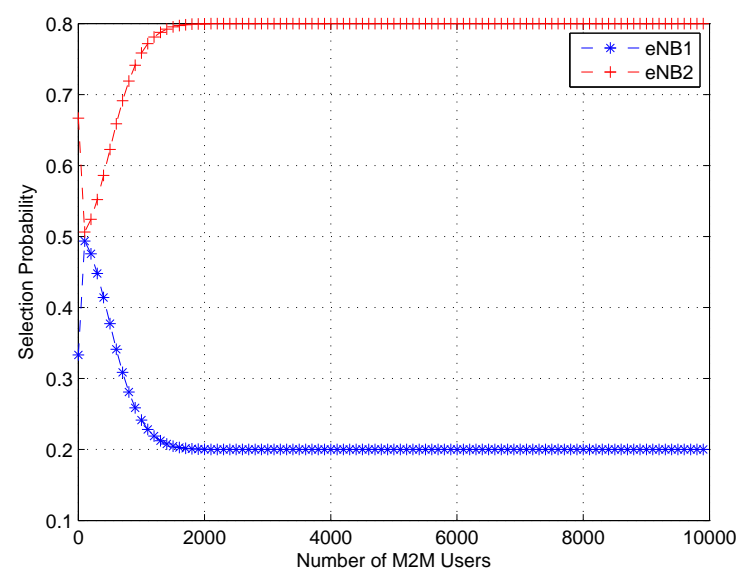

(b) Selection probability (initially $\alpha_{1}=\alpha_{2}$ ).

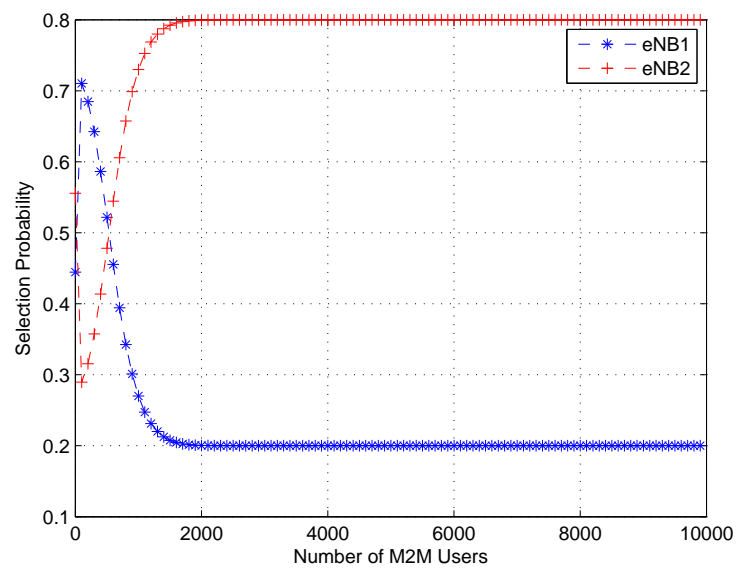

(d) Selection probability (initially $\alpha_{1}>\alpha_{2}$ ).

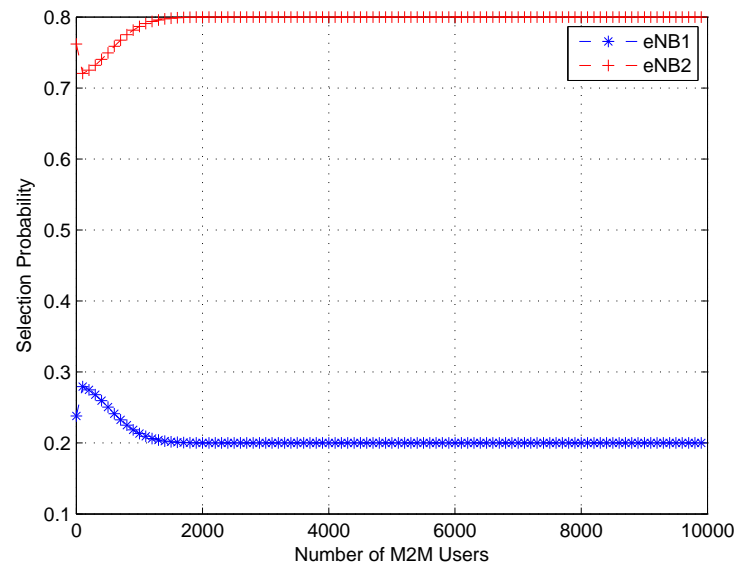

(f) Selection probability (initially $\alpha_{1}<\alpha_{2}$ ).

Figure 4.12: Throughput and selection probability of eNBs when applying dynamic ACB with $F T R_{1}<F T R_{2}$ 


\subsubsection{Group three: $F T R_{1}<F T R_{2}$}

As opposite to the group two, here we consider $e N B_{2}$ with higher transmission rate than $e N B_{1}$. The three cases of this group are:

-Case 3(a): Both base stations consider equal barring parameter but different transmission rates $(F T R 1<F T R 2)$, as shown in Figures 4.12(a) and 4.12(b).

-Case 3(b): The ACB parameter of base station one is higher than that of base station two (e.g., $\alpha_{1}>\alpha_{2}$ ), as shown in Figures 4.12(c) and 4.12(d).

-Case 3(c): Figures 4.12(e) and 4.12(f) show the results of this case. In this case, the base station two sets higher ACB parameter than one, (e.g., $\alpha_{1}<\alpha_{2}$ ).

In general, Figures 4.12(a), 4.12(c) and 4.12(e) show the results of load balancing method. It can be observed in Figure 4.12 that $e N B_{2}$ shows the best performance. Also, the $e N B_{1}$ throughput increases significantly after applying dynamic ACB method. This is due to the fact that frame transmission rate and dynamic access control method distribute the traffic among base stations and improve the overall system performance and access success probability among base stations as well as avoid overload situation in a particular base station. In this group, the selection probability of $e N B_{2}$ shows higher value than $e N B_{1}$, that is depicted in Figures 4.12(b), 4.12(d) and 4.12(f). In the dynamic ACB method, the decision rule of selecting a base station among these three groups is summarized in Table 4.2. If the base station supports same transmission rate, M2M device can choose any base station after applying the dynamic ACB method. Otherwise, device can choose a base station depending on the highest FTR regardless of the initial ACB parameter.

Table 4.2: Simulation results of selecting probability

\begin{tabular}{|l|c|c|c|}
\hline $\begin{array}{l}\text { ACB } \\
\text { parameter }(\alpha)\end{array}$ & $\begin{array}{c}\text { Group One } \\
\left(F T R_{1}=F T R_{2}\right)\end{array}$ & $\begin{array}{c}\text { Group Two } \\
\left(F T R_{1}>F T R_{2}\right)\end{array}$ & $\begin{array}{c}\text { Group Three } \\
\left(F T R_{1}<F T R_{2}\right)\end{array}$ \\
\hline$\alpha_{1}=\alpha_{2}$ & either & $e N B_{1}$ & $e N B_{2}$ \\
\hline$\alpha_{1}>\alpha_{2}$ & either & $e N B_{1}$ & $e N B_{2}$ \\
\hline$\alpha_{1}<\alpha_{2}$ & either & $e N B_{1}$ & $e N B_{2}$ \\
\hline
\end{tabular}


In the next chapter, we present and evaluate our proposed relay assisted resource allocation method within a single cell system. 


\section{Chapter 5}

\section{Relay Assisted Radio Resource}

\section{Allocation Method for MTC Network}

In this chapter, firstly, we present a background study of radio resource allocation methods which are applicable for MTC systems. Then, we propose and evaluate a relay assisted resource allocation method for MTC by considering multi-user, single-cell system in which H2H, MTC device and relay nodes co-exist together.

\subsection{Previous works}

In MTC-enabled LTE network, signalling congestion arises in uplink transmission when a large number of devices send connection requests simultaneously. When MTC devices send their requests directly to the LTE network, radio resource management (e.g. resource allocation and scheduling) of LTE needs to consider several factors machine-type communication such as energy, QoS requirements, delay constraints etc. The authors in [4], propose energy efficient radio resource allocation method for MTC-enabled LTE networks in which they model resource allocation problem as sum power minimization problem. Similarly, the authors in [47] propose a max-utility scheduling method for machine-type communication by considering QoS requirements as minimizing co-channel interference between $\mathrm{H} 2 \mathrm{H}$ and MTC devices. Group based allocation [5] and joint access control and allocation method [48] consider fairness and random access efficiency in their allocation procedure. The above mentioned methods choose allocation 
metric as their chosen application perspective. For example, joint access and radio resource allocation method considers delay sensitive application, similarly group based allocation method considers group of smart metering devices. Two important factors need to be considered in the design of radio resource allocation method for MTC-enabled LTE network; first one, the QoS requirements of LTE users should not be hampered by the allocation method, secondly, influence factor of allocation methods should be the data rate of the network. In the proposed relay assisted radio resource allocation method, we consider those two factors. Firstly, we allocate radio resource to the normal LTE users by ensuring their QoS requirements, then allocate resource blocks (RBs) to the requested MTC devices with the help of relay node if required. In the next section, we describe the basic allocation element that is the radio resource block structure in the LTE frame.

\subsection{Radio resource block}

In LTE, single-carrier frequency division multiple access (SC-FDMA) is used as a uplink access scheme which is basically discrete Fourier transform pre-coded orthogonal frequency division multiple access method (DFT-spread OFDM). Opposite to OFDM, the SC-FDMA scheme supports lower peak-to-average power ratio (PAPR) which is suitable for power efficiency in uplink transmission. In order to support lower peak-to-average power ratio, RBs are assigned into contiguous pattern.

In LTE, uplink and downlink channels are divided into a number of sub-channels which comprise of time-frequency resources, known as radio resource blocks (RBs), which are distributed to the users upon the access requests. Figure 5.1 shows the LTE radio frame and radio resource block structure. Each radio frame duration is $10 \mathrm{~ms}$ and it consists of 20 slots, each with $0.5 \mathrm{~ms}$ or 10 subframes. In the time domain, radio resources are allocated in every transmission time interval (TTI) having 2 slots, each with $0.5 \mathrm{~ms}$, and hence of $1 \mathrm{~ms}$ duration. In the frequency domain, radio resources are allocated into sub-channels. Each sub-channel comprises of 12 subcarriers having $15 \mathrm{kHz}$ spacing. More specifically, radio resource blocks are represented by symbols in the time domain and subcarriers in the frequency domain. The range of RBs depends on the available bandwidth. According to [34] [49], the number of RBs ranges from 6 to 110. Table 


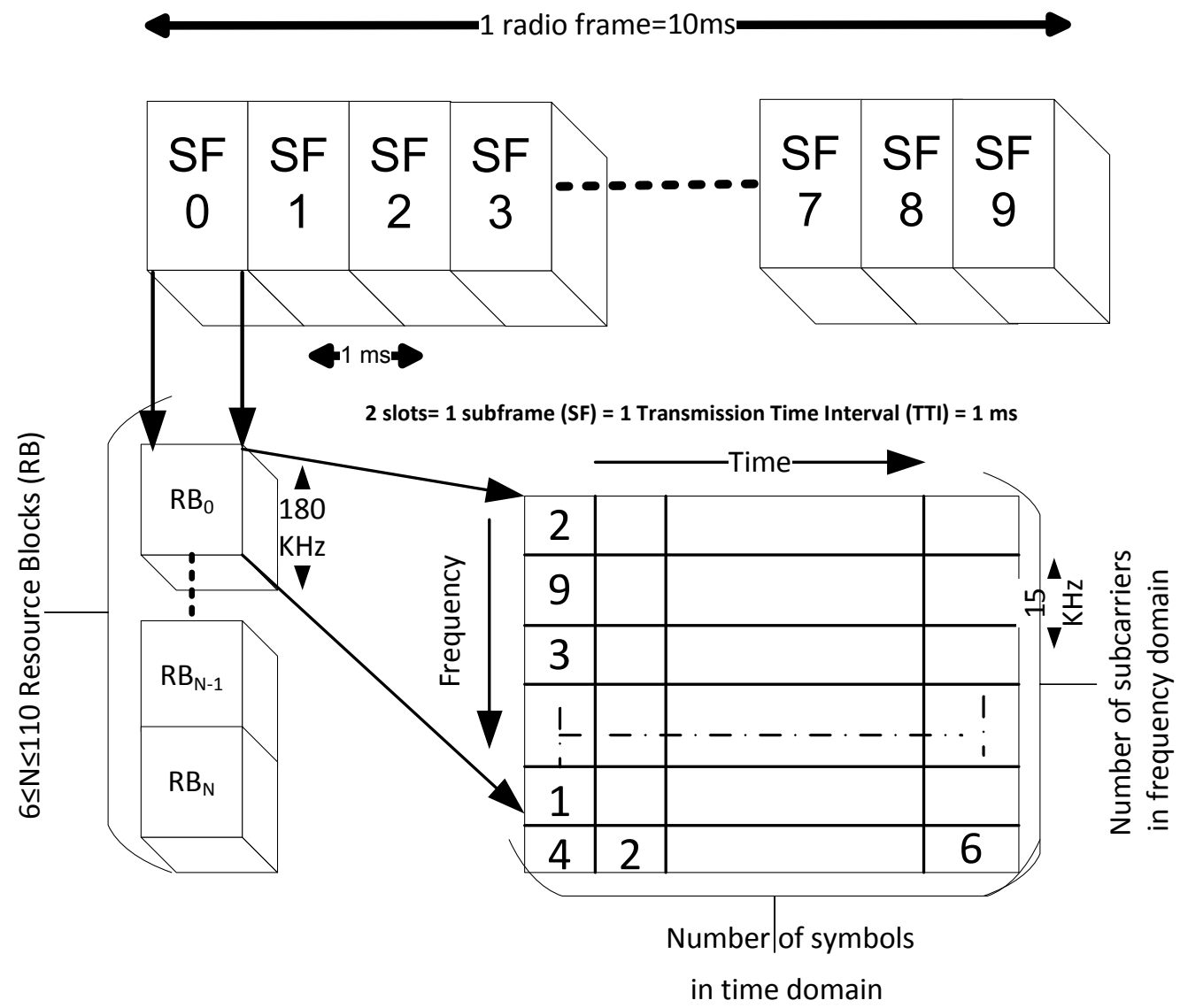

Figure 5.1: The LTE frame and radio resource block structure.

5.1 shows that the number of resources blocks increase with the increase of channel bandwidth. The proposed relay assisted radio resource scheduling method is discussed in the next section.

\subsection{Relay assisted radio resource allocation method}

\subsubsection{System model}

We consider a multi-user, single-cell system as our system model, as shown in Figure 5.2. This system is regarded as a LTE-Advance single-cell system and it supports one base station, fixed number of relay nodes, multiple MTC devices and LTE user equipments (UEs). The MTC device communicates to the MTC server through the base station using either direct link or relay link depending on the channel information. The base station/eNodeB (BS/eNB) maintains a single time slotted buffer in which access requests are queued until BS allocates radio resources to each 
Table 5.1: Channel bandwidth and resource block in LTE.

\begin{tabular}{|c|c|}
\hline Channel Bandwidth $(\mathrm{MHz})(W)$ & Number of Resource Block $(\mathbf{R B})(\eta)$ \\
\hline 1.4 & 6 \\
\hline 3 & 15 \\
\hline 5 & 25 \\
\hline 10 & 50 \\
\hline 15 & 75 \\
\hline 20 & 100 \\
\hline
\end{tabular}

request.

\subsubsection{Problem formulation}

Consider a single-cell system that supports $l=\{1,2, \ldots L\}$ number of LTE users, $m=\{1,2, \ldots M\}$ number of MTC devices and fixed number of relay nodes. Here, we consider the number of relay nodes $(\mathrm{RN})$ is $R$ (i.e. $R N=\{1,2 \ldots R\})$. The MTC device generates a small amount of data. In the transmission time interval (TTI), MTC devices send and receive data to/from the BS/eNB. One TTI corresponds to one-time slot in the buffer. The duration of each time slot in the buffer is $1 \mathrm{~ms}$.

Let the total number of time slots in the buffer be $\aleph$ (i.e. $\left.\left\{T T I_{1}, T T I_{2} \ldots . T T I_{\aleph}\right\}\right)$ and transmission bandwidth be $W$. According to Table 5.1, the total number of resource blocks will be $T_{R B}=2 \times \aleph<\eta$, where $\eta$ denotes the maximum number of resource block supported by $W$. For example, if the available bandwidth is $W=5 \mathrm{MHz}$, then the total RBs will be $T_{R B}=2 \times \aleph<25$. The base station serves the number of $D_{n}$ at time slot $T T I_{n}$, where $1 \leq n \leq \aleph$. At the beginning of the $T T I_{n}$, assume that there are $\theta_{n}$ number of time slots in the queue occupied by other requests and the base station receives requests from $M_{i_{n}}(i \in l)$ MTC devices and $L_{j_{n}}(j \in l)$ LTE users at time slot $T T I_{n}$, where $1 \leq n \leq \aleph$. Here, we consider two consecutive RBs as a scheduling block to be allocated to the LTE users and one RB for MTC user. The expected number of available RBs at the beginning of $T T I_{n+1}$ will be

$$
\theta_{n+1}=T_{R B}-\theta_{n} \times 2-\left(\text { total no. of allocated RBs at } T T I_{n}\right)+\left(\text { total no. of released RBs at } T T I_{n}\right) .
$$




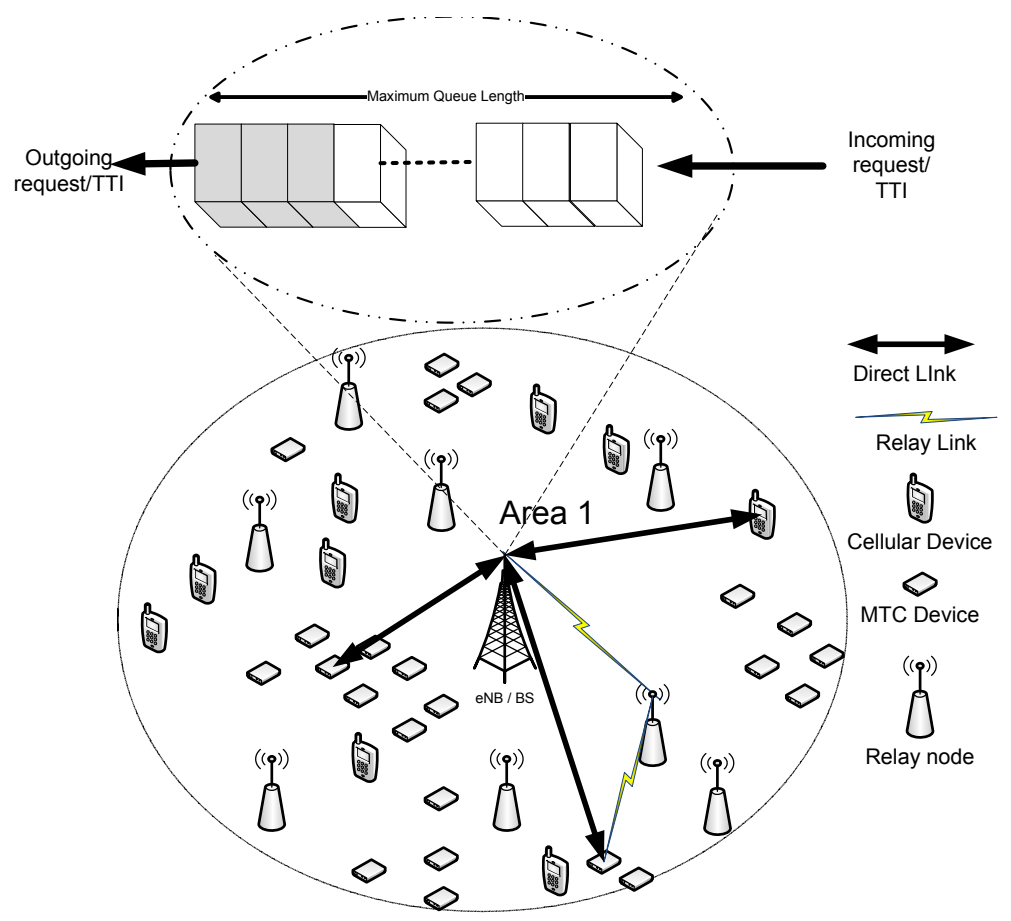

Figure 5.2: System model of relay assisted M2M communication.

That is,

$$
\theta_{n+1}=2 \times \aleph-2 \times \theta_{n}-\left(M_{i_{n}}+L_{j_{n}} \times 2\right)+2 \times D_{n} .
$$

The overflow problem occurs when the expected number of RBs is greater than the available RBs in the buffer. That is,

$$
\theta_{n+1}>2 \times\left(\aleph-\theta_{n}\right) \text { and } \theta_{n+1}>\eta
$$

To control this overflow, we design a allocation method which at first assigns RBs to the LTE users, then calculates the expected number of available RBs for MTC devices. In this case, at $T T I_{n+1}$, the expected number of available RBs for MTC device will be,

$$
\Lambda_{n+1}=2\left(\aleph-\theta_{n}\right)-L_{j_{n}} \times 2+D_{n} \times 2
$$

There are two possible situations that can occur. In the first case, there are enough resource blocks available to satisfy all MTC device requests. In the second case, only few devices can get access to the RBs. That is, 


$$
\text { Case 1: } \Lambda_{n+1} \geq M_{n} \text {, }
$$

and

$$
\text { Case 2: } \Lambda_{n+1}<M_{n} \text {. }
$$

In these two cases, we discuss the proposed resource allocation method in the next section.

\subsubsection{Relay assisted radio resource allocation method}

In Case 1, the objective function is to maximize the MTC channel capacity of the base station. In this case, all users along with the low signal-to-noise (SNR) devices get radio resources but it does not improve channel capacity of the base station. Before allocating the resource blocks, the BS measures SNR all of its MTC links. According to [50], we assume that the direct link of

the MTC devices experience independent fading and the channel capacity $\left(C_{k}\right)$, the SNR $\left(\gamma_{k}\right)$ over the direct link of the $k$ th MTC device is given as

$$
\gamma_{k}=\frac{P_{k}}{\sigma^{2}}\left|h_{k}\right|^{2},
$$

and

$$
C_{k}=W \log \left(1+\frac{P_{k}}{\sigma^{2}}\left|h_{k}\right|^{2}\right),
$$

where $h_{k}$ is the channel gain of the direct link and $\sigma^{2}$ is the noise power. $P_{k}$ is the transmitting power of the $k$ th MTC device. In order to get the maximum channel capacity, the following constraint must be satisfied, that is

$$
\begin{gathered}
\gamma_{k} \geq \tau^{t h}, \\
\tau^{t h}=\frac{1}{L} \sum_{l=1}^{l=L} \gamma_{l} .
\end{gathered}
$$

Here, we consider the threshold value as the average received SNR of the LTE users. This constraint works for two purposes. Firstly, we can identify the low SNR devices which need relay channel. Secondly, using this constraint, the BS fairly assigns relay nodes among MTC channels. Here, we assume that the MTC devices use the amplify-forward (AF) relay channel. In AF mode, the BS $b$, the MTC device $k$ and the relay node $r$ experience flat fading channel with channel gain $h_{r b}$ and $h_{k r}$ respectively. According to [49], the SNR between device and relay 
node, relay and BS is $\gamma_{k r}=\frac{P_{k}}{\sigma^{2}}\left|h_{k r}\right|^{2}$, and $\gamma_{r b}=\frac{P_{r}}{\sigma^{2}}\left|h_{r b}\right|^{2}$ respectively. The achievable capacity of the relay channel $\left(C_{k r}\right)$ is given as:

$$
\begin{array}{r}
C_{k r}=\frac{W}{2} \log \left(1+\gamma_{k}+\frac{\gamma_{k r} \cdot \gamma_{r b}}{\gamma_{k r}+\gamma_{r b}+1}\right) \\
=\frac{W}{2} \log \left(1+\frac{P_{k}}{\sigma^{2}}\left|h_{k}\right|^{2}+\frac{P_{k}\left|h_{k r}\right|^{2} P_{r}\left|h_{r b}\right|^{2}}{P_{k} \sigma^{2}\left|h_{k r}\right|^{2}+P_{r} \sigma_{r}^{2}\left|h_{r b}\right|^{2}+\sigma_{r}^{2} \sigma^{2}}\right) .
\end{array}
$$

In Case 1, the base station assigns relay node to the MTC devices that do not satisfy the constraints (i.e., (5.8)) and also the relay link capacity shows higher value than direct link. That is, the MTC device $k$ selects relay $r$ when

$$
\gamma_{k}<\tau^{t h} \text { and } C_{k r}>C_{k} \text { where } r \in R N
$$

In our proposed method, the BS allocates RBs to the MTC devices with the objective to maximize the overall MTC channel capacity.

$$
C_{M}=\sum_{k=1}^{M} \max \left(C_{k}, C_{k r}\right)
$$

In Case 2, (5.5) refers to the fact that the number of available RBs is lower than the number of access requests. In this case, the available RBs are allocated only to the highest SNR devices.

\subsubsection{Relay assisted radio resource allocation algorithm (RARRA)}

The RARRA works on a single cell system that supports one base station with the time slotted buffer $\left.\left\{T T I_{1}, T T I_{2} \ldots T T I_{\aleph}\right\}\right)$. Assuming that this system supports $l=\{1,2, \ldots L\}$ number of LTE users, $m=\{1,2, \ldots M\}$ number of MTC devices and the $R$ number of relay node $(R N)$ (i.e. $R N=\{1,2 \ldots R\})$. The detail flow diagram of relay assisted radio resource allocation (RARRA) algorithm is shown in Figure 5.3. It works as follows:

Step 1: The base station receives requests from $L_{n}\left(L_{n} \in l\right)$ LTE users and $M_{n}\left(M_{n} \in m\right)$ MTC users at $T T I_{n}$. 


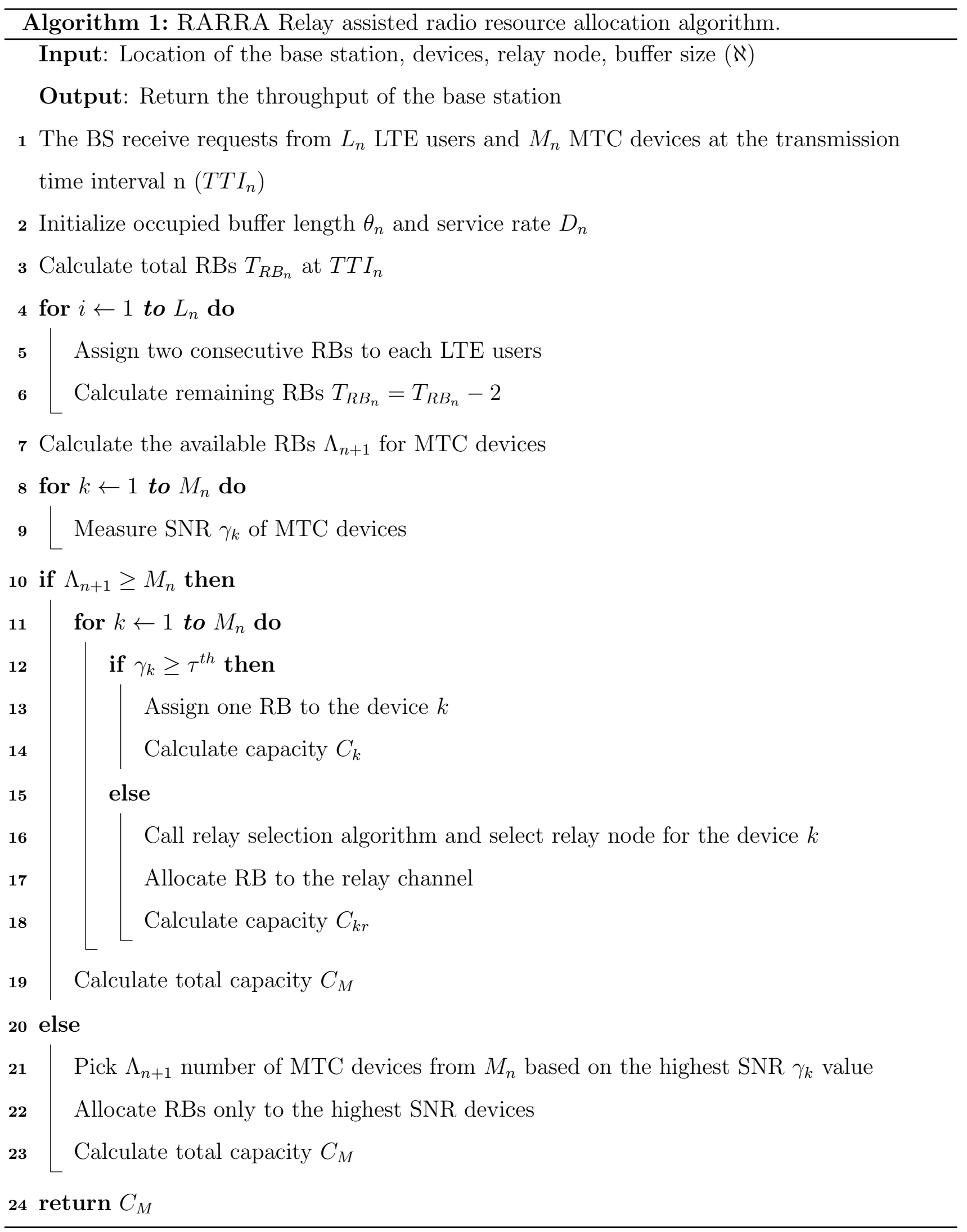


Step 2: To ensure QoS requirements, at first, the base station allocates RBs to the LTE users. Then, it calculates the expected number of available RBs $\left(\Lambda_{n+1}\right)$ for the MTC device. In this step, the BS also measures $\operatorname{SNR}\left(\gamma_{k}\right.$ where $\left.k=1,2 \ldots M_{n}\right)$ of every MTC channels.

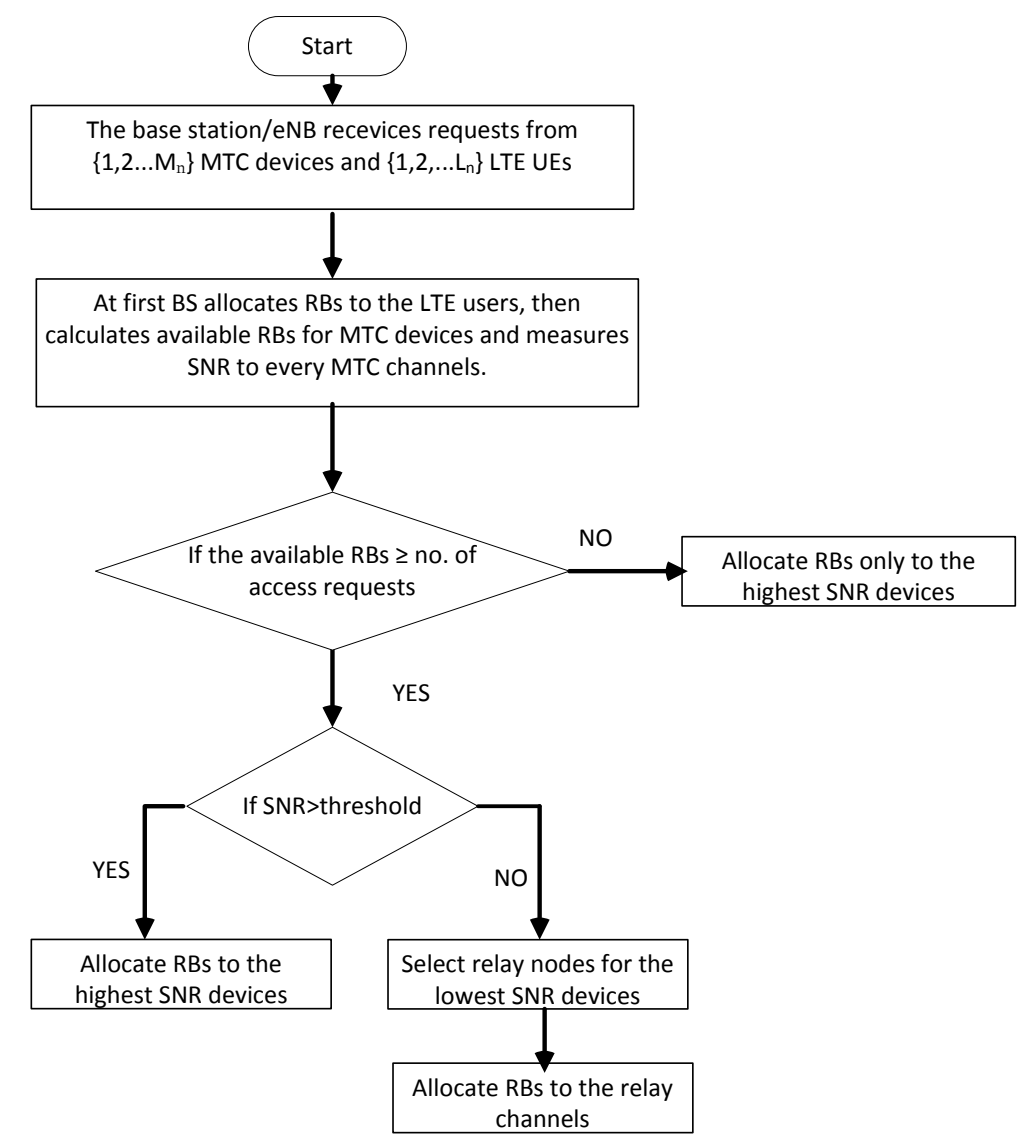

Figure 5.3: Flow chart of radio resource allocation method.

Step 3: The BS checks the condition of available RBs. That is, $\Lambda_{n+1} \geq M_{n}$ in line 10 of Algorithm 1.

- Case 1 of the RARRA starts when this condition becomes true. Lines 11 to 19 in the Algorithm 1 refer to Case 1.

- In this case, the BS checks the SNR of the device to threshold value $\gamma_{k} \geq \tau^{t h}$ in line 12. If any device satisfies this condition, the BS immediately allocates RBs to it.

- Otherwise, the BS selects relay node $R$ from the set of the relay nodes $R N$ (i.e. $R \in R N$ ) and allocates RBs to the relay channel. Here, we use source-based Bayes 
amplify-forward relay (BRA-SAF) technique [50] to choose relay node for the MTC device.

- Case 2 (Lines 21-23) refers to the overflow case in which the number of access requests is higher than the number of available radio resources. In this case, the RARRA allocates RBs to the devices depending on the highest received SNR value. To control the overflow problem, the BS can apply the existing congestion control methods. For example, access class barring methods (ACB, EAB) or proposed dynamic ACB (DACB) methods can be used to solve the overflow problem.

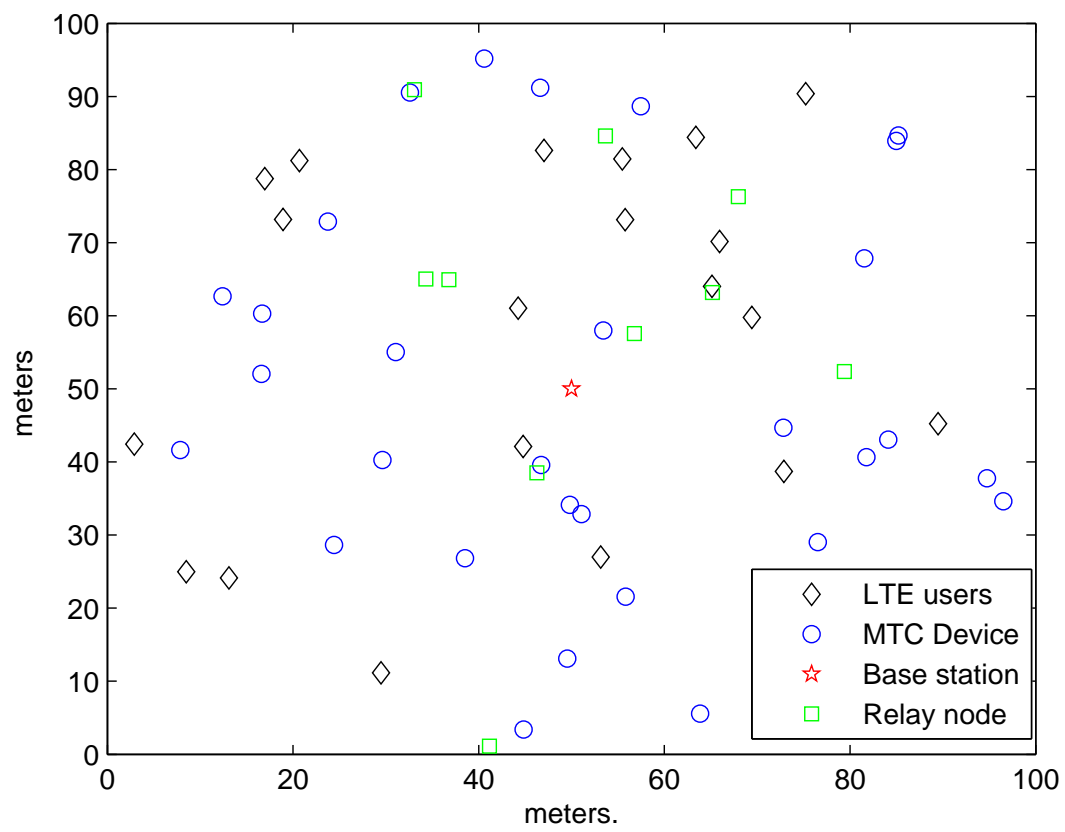

Figure 5.4: Simulation model of relay assisted M2M communication.

\subsection{Performance analysis of RARRA algorithm}

For the numerical evaluation of the RARRA algorithm, we consider a single cell system, as illustrated in Figure 5.4, where $L$ number of LTE users, $M$ number of M2M devices and $R$ number of relay nodes are distributed randomly within $50 \mathrm{~m}$ radius of the base station. All the communications between M2M device and M2M users are done through the base station. 
For uplink and downlink communication, the base station allocates radio resource to all of its active channels. Here, the simulation model is based on a single-cell LTE system having $20 \mathrm{MHz}$ available bandwidth. All other simulation parameters and values for RARRA algorithm are shown in Table 5.2 .

Table 5.2: Simulation Parameters for RARRA.

\begin{tabular}{|c|c|}
\hline Parameters & Values \\
\hline System bandwidth $(W)$ & $20 \mathrm{MHz}$ \\
\hline Cell radius & $50 \mathrm{~m}$ \\
\hline TTI duration & $1 \mathrm{~ms}$ \\
\hline Buffer size $(\aleph)$ & $30-50$ TTI \\
\hline Simulation time & 100 TTI \\
\hline Number of LTE users $(L)$ & $20-30$ \\
\hline Number of relay node $(R)$ & 10 \\
\hline Number of MTC users $(M)$ & $30-400$ \\
\hline Arrival rate $(\lambda)$ & 0.2 \\
\hline Relay technique & Amplify-forward \\
\hline Path-loss exponent & 4 \\
\hline
\end{tabular}

The objective of RARRA is to maximize the system capacity of the base station as well as to increase the access success probability of the M2M devices. To ensure QoS performance of LTE users, firstly RBs are allocated to the LTE users, then RARRA estimates available RBs for MTC devices. In RARRA method, the available RBs for MTC depend on buffer size and service rate of the base station. The impact of the buffer size to the success probability is analyzed in the next section.

\subsubsection{Impact of buffer size $(\aleph)$}

Here, the base station maintains a finite length of time slotted buffer. Each slot of the buffer is visualized as 1ms transmission time interval (TTI). In an LTE network, the relation between TTI and resource block (RB) is shown in Figure 5.1. Resources are allocated upon receiving 
requests into each TTI. Here, we assume that the total available RBs of the base station depends on transmission bandwidth and the buffer size, that is, how many TTI the buffer supports. Equation (5.3) refers to the available RBs for M2M device at $(n+1)$ transmission time interval. We evaluate this equation with different buffer lengths. Figure 5.5 shows the access success

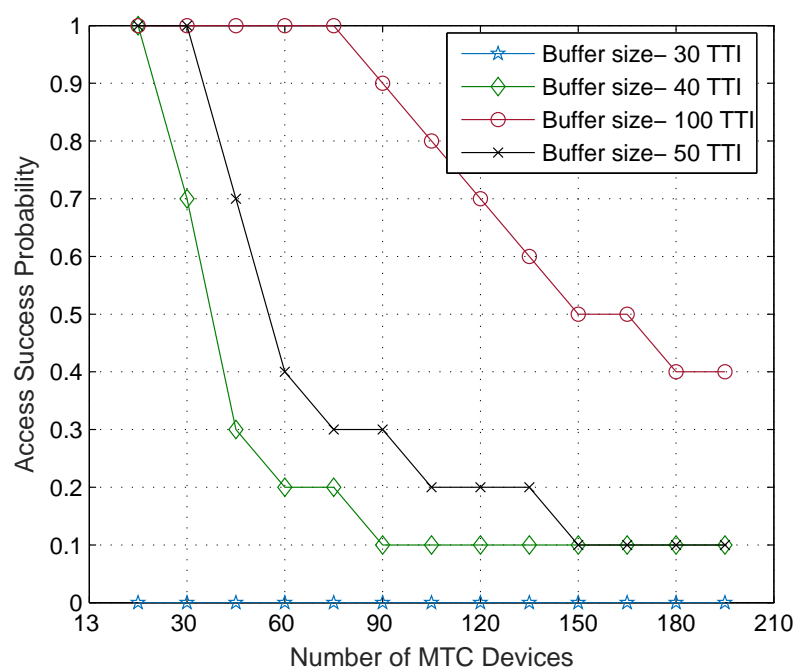

(a) Access success probability

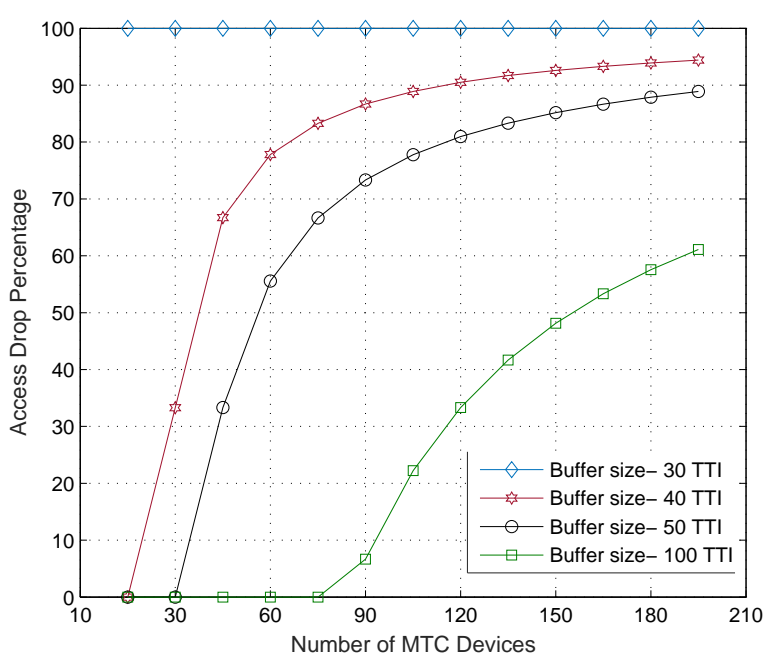

(b) Access drop percentage

Figure 5.5: Impact of buffer size when available bandwidth $W=20 \mathrm{MHz}$ and $L=30$.

probability and the percentage of access drop of M2M devices with different buffer size. It can be observed from this figure that the access success probability of M2M devices is highly influenced by buffer length. According to the Table 5.1, the upper limit of buffer length depends on channel bandwidth. For example, for $20 \mathrm{MHz}$ bandwidth, the base station can support up to 50 TTI buffer size to allocate 100 physical resource blocks among users. For $20 \mathrm{MHz}$ bandwidth, if the buffer length is $\aleph=30$ TTI, in this case, the base station allocates resource blocks only to the LTE users (e.g 30 LTE users). If buffer length increase by 40 TTI, the base station can support 10 MTC devices whereas when $\aleph=50 \mathrm{TTI}$, the base station supports around $30 \mathrm{M} 2 \mathrm{M}$ devices along with 100 percent access success probability to 30 LTE users. Similar factor is shown in Figure 5.5(b), with 50 TTI buffer length, the access drop percentage is nearly zero upto 30 users. The general observation from these Figures 5.5(a) and 5.5(b) is that the access drop percentage decreases, and access success probability increases with the increase in buffer size. 
To evaluate the performance of RARRA method, we choose buffer size 50 TTI for Case 1 and 30 TII for Case 2 respectively. The analysis of system throughput of RARRA method is presented in the next section.

\subsubsection{Case 1: $\Lambda_{n+1} \geq M_{n}$}

In RARRA method, the relay assisted resource allocation works when base station has enough radio resource to serve all MTC device requests. Here, we consider $30 \mathrm{M} 2 \mathrm{M}$ devices and $20 \mathrm{LTE}$ users and 20 relay nodes for buffer size 50 TTI of $20 \mathrm{MHz}$ bandwidth. The threshold value $\left(\tau^{t h}\right)$ of the RARRA method is set as average received SNR of the LTE users. Figure 5.6(a) shows the overall improvement of the MTC channel capacity with relay assisted resource allocation method. Similarly, Figure 5.6(b) shows the improvement of each MTC user capacity with and without assisted by relay node. The impact of threshold value in the MTC channel capacity is shown in Figures 5.7 and 5.8. It can be seen from Figure 5.6(b) that around 25 out of 30 users use relay node when the average received SNR of the LTE users consider as a threshold value, (e.g. Equation (5.9)). This is due to the fact that maximum number of M2M devices in our simulation model are located between middle and boundary area of the network, as shown in Figure 5.4. In this scenario, if the average received SNR considers as a threshold value, the base station can assign maximum number of relay nodes to the maximum number of MTC device, as shown in Figures 5.6 and 5.7. When threshold value is set as minimum received SNR, the base station picks only edge node devices to assign relay node. In this case, three users (e.g. users 9, 10 and 17 in Figure 5.7) out of thirty can choose relay node to improve their capacity. Similar fact is observed in maximum received SNR case, in which devices located besides base station choose relay node which is practically an infeasible case.

Finally, the aggregated throughput of the base station is analyzed with three different threshold value. Figure 5.8 shows that in RARRA method, when there are enough radio resources available to serve MTC access requests, the base station can improve aggregated throughput by considering average received SNR as a threshold value to assign relay node to the MTC device. However, this decision factor is influenced by many other factors, such as the relative location of MTC device and base station, how many relay nodes the base station can support, and which 


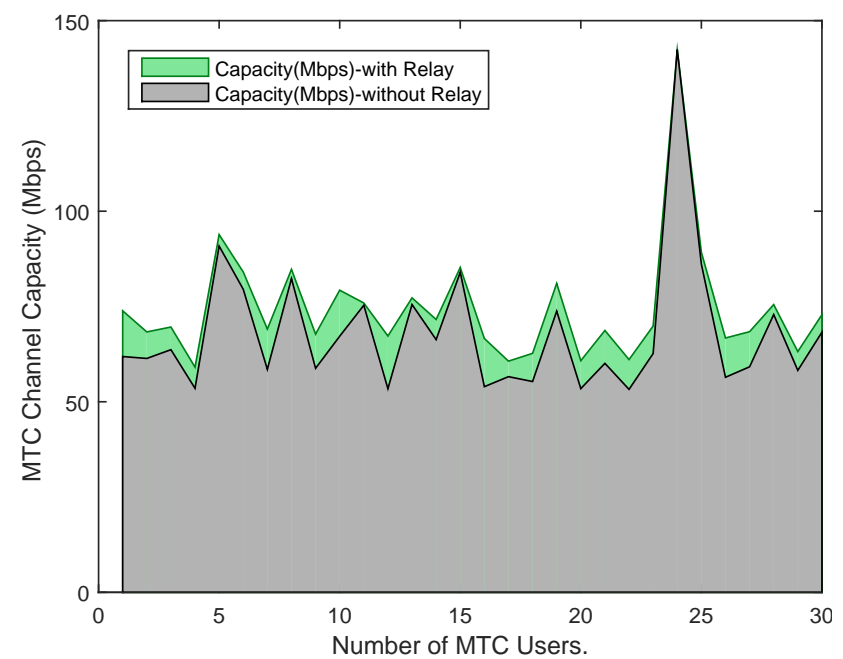

(a) Overall MTC channel capacity with/without re-

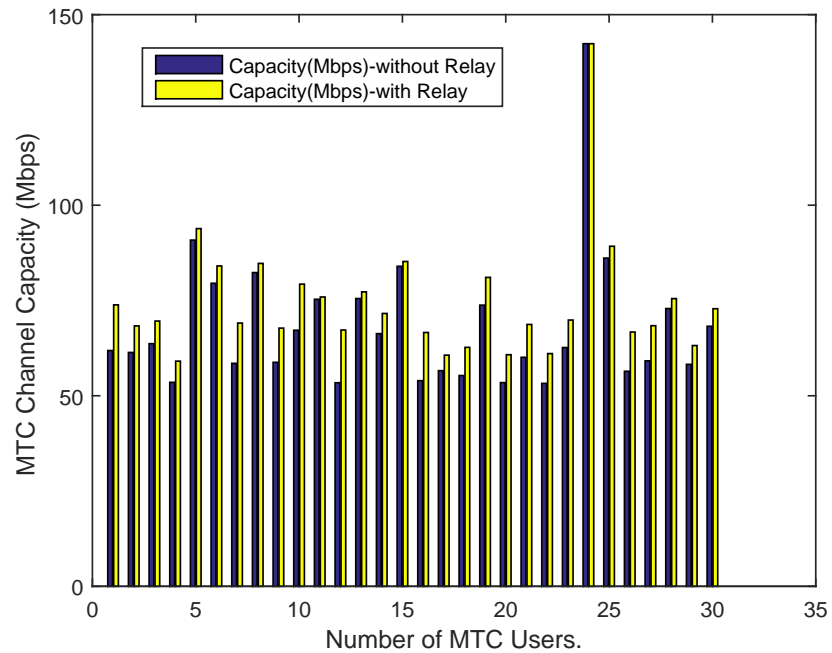

(b) MTC channel capacity per users

lay node.

Figure 5.6: MTC channel capacity when $\Lambda_{n+1} \geq M_{n}, \tau^{t h} \doteq \frac{1}{L} \sum_{l=1}^{l=L} \gamma_{l}, \aleph=50 \mathrm{TTI}$ and $W=20 \mathrm{MHz}$.

relay selection technique is used etc.

\subsubsection{Case 2: $\Lambda_{n+1}<M_{n}$}

Case 2 refers to the situation when the number of MTC access requests are larger than available radio resources. In this case, the RARRA method assigns RBs only to the those MTC devices which has highest SNR value. It can be observed from Figure 5.9 that the base station assigns RBs only to the 15 users out of 30 . Here, 50 percent users get RBs when buffer size sets to 30 TTI and the number of users access requests for both MTC and LTE consider as 50. However, in this situation, if the number of users access requests increase, then the percentage of access drop in the base station will increase rapidly. Consequently, this will affect the overall throughput of the base station. This problem is regarded as overload problem in radio access network (RAN). The possible solution for resolve this problem will be: i) the base station can increase the buffer size when percentage of access drop becomes 20 to 50 percent, ii) the base station can apply the access class barring method such as ACB, EAB, cooperative ACB to reduce the number of MTC access requests, iii) the base station allocates separate radio resources for MTC device. 


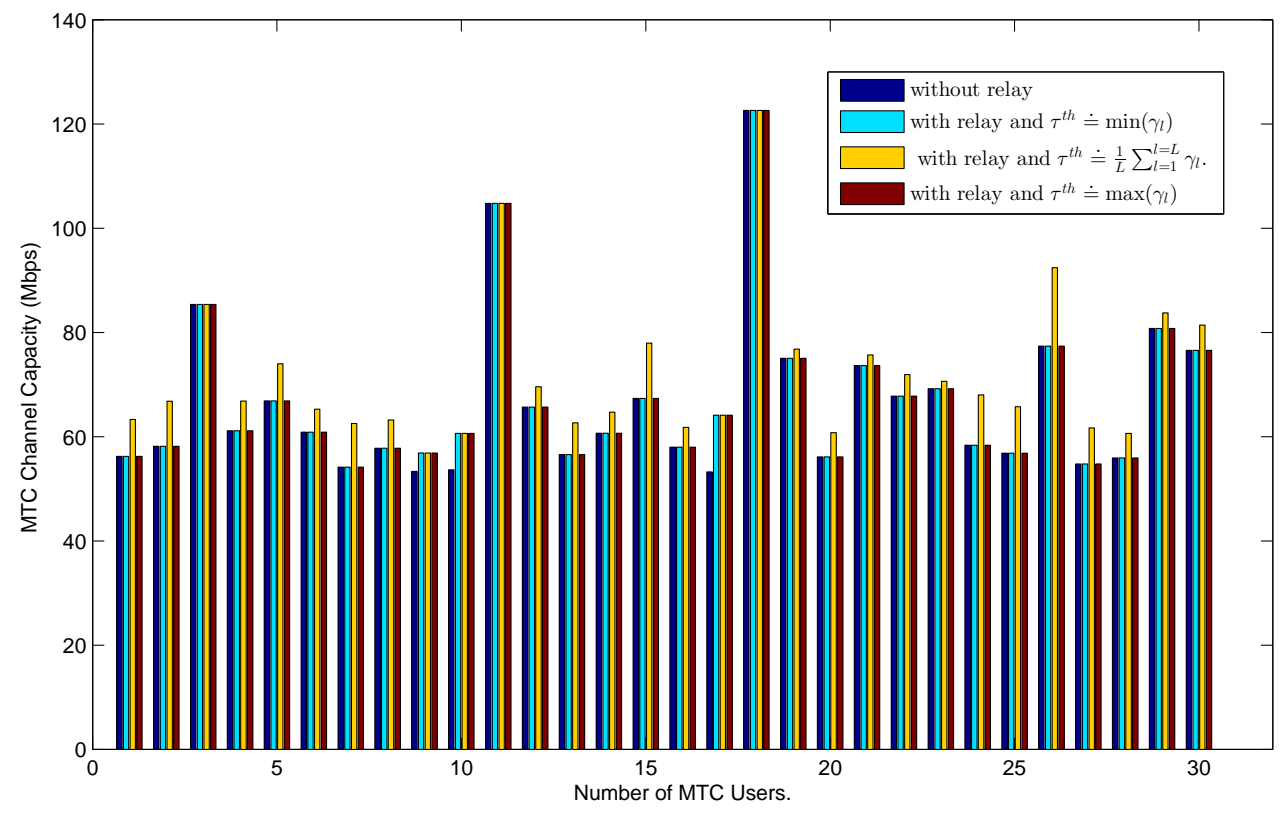

Figure 5.7: The MTC channel capacity with different threshold values.

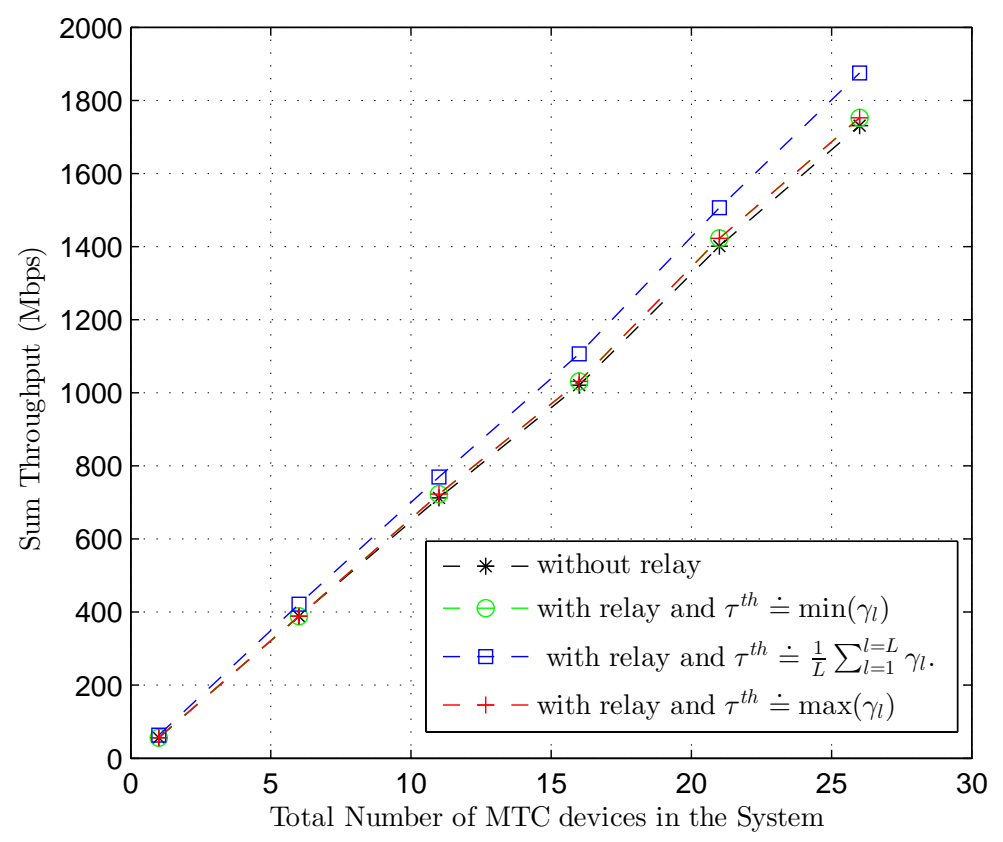

Figure 5.8: Performance analysis of RARRA in term of sum throughput considering three different threshold values. 


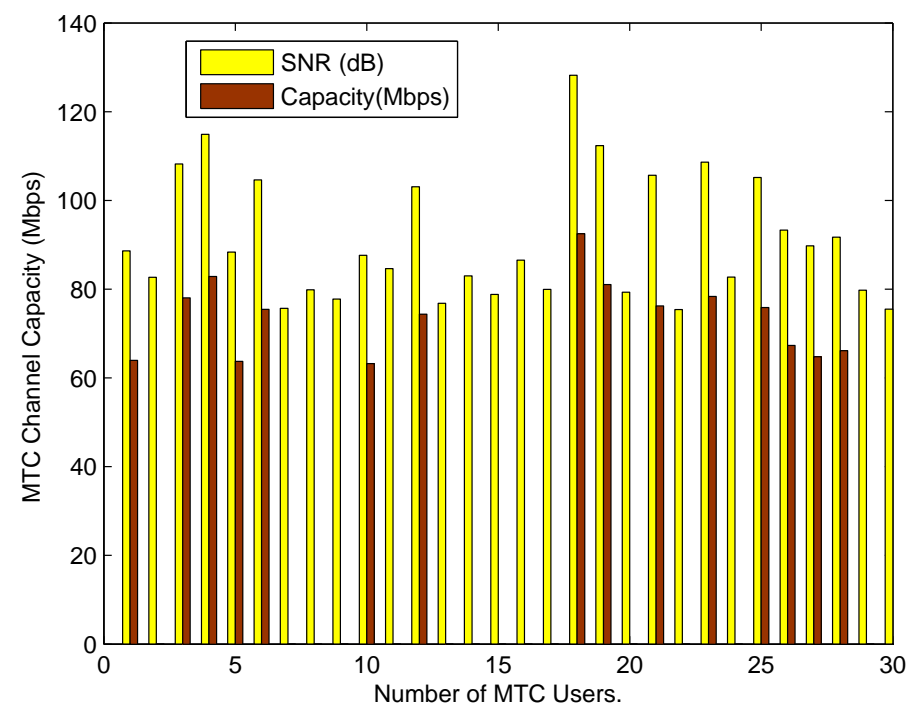

Figure 5.9: MTC channel capacity when $\Lambda_{n+1}<M_{n}$, $\aleph=30$ TTI and $W=20 \mathrm{MHz}$.

The various RAN based overload control solution is discussed in Chapter 3.

In the next chapter, we present the summary of proposed dynamic ACB and RARRA methods by identifying their future improvements. 


\section{Chapter 6}

\section{Conclusions and Future work}

This thesis mainly focused on the problem of massive access requests of cellular M2M systems. Two solution methods have been proposed and evaluated in this thesis. First method is the dynamic access class barring which prevents random access channel congestion. Second one is relay-assisted radio resource allocation method which maximizes the sum throughput of the system by assigning relay nodes and radio resources to the M2M devices. In this chapter, we present the main features and scope of improvements of these two methods.

\subsection{Conclusion}

The proposed dynamic access class barring (DACB) method balances massive access requests among base stations and, at the same time by using this method, device can choose appropriate base station. The distinguishable features of this method are:

- The DACB method has three parts: load balancing, eNB selection and random access.

- The DACB utilizes each base station access success probability as an access barring parameter and this parameter is used in load balancing procedure.

- In this method, each device chooses base station based on maximum aposterior probability (MAP) value in which previous access success probability works as a prior knowledge.

- In the random access process, devices apply the same access class barring method. 
Numerical results show that the proposed dynamic access control method distributes traffic among base stations and improves overall throughput and access success probability among base stations as well as avoid overload situation in a particular base station. Moreover, each device can apply posterior probability based base station selection method to select low load base stations which consequently reduce radio access network congestion. However, this method shows some limitations which can be directed as future work. The main limitations of this method are:

- The DACB method is applicable for those devices that can choose more than one network. Only the edge node devices can apply this method to choose appropriate base station.

- Similar to the ACB method, the DACB method restricts all types of MTC traffic.

- For practical application of this method, the MTC device needs some level of intelligence to apply MAP based selection method.

The second proposed solution is radio resource allocation method (RARRA) in which relay nodes help low SNR device to send their access requests. The main key points of this method can be summarized as follows:

- The main objective of this method is to maximize the sum throughput of the base station.

- In RARRA method, an analytical model has been proposed to estimate available resource blocks for M2M devices.

- Relay based allocation method works when the available resource blocks of the base station can accommodate M2M traffic.

Numerical results show that the buffer size influences the access success probability of M2M traffic. The performance of the base station becomes maximum when average received SNR value is considered as a threshold value. However, the RARRA does not work in the buffer overflow situation. When buffer overflow occurs, the base station assigns resource blocks only to the highest SNR devices. 


\subsection{Future Work}

It would be interesting to investigate the aforementioned limitations of DACB and RARRA methods. The analytical model of the eNB selection is derived based on only one metric that is access success probability. Some other factors can be considered, for example QoS requirements, power consumption, delay on eNB selection. Different parameters can be considered instead of just selecting the low load base stations. Similarly, other scheduling parameters can be investigated in RARRA instead of data rate of the base station. The RARRA method works when there are adequate resources available to serve M2M requests and detect overflow situation. Resolving this buffer overflow problem will be another new possibility of research direction. 


\section{Bibliography}

[1] C. Y. H. Chie Ming Chou, "Loading Prediction and Barring Controls for Machine Type Communication," IEEE ICC 2013: Wireless Communication, 2013.

[2] T. Taleb and A. Kunz, "Machine type communications in 3GPP networks:potentials,challenges, and solutions," IEEE Wireless Communication Magazine, pp. 178-184, March 2012.

[3] 3GPP TR 23.888 V1.3.10, "System Improvements for Machine-Type Communications," June 2011.

[4] A. Aijaz and A. H. Aghvami, "On Radio Resource Allocation in LTE networks with Machine-to-Machine Communications," Vehicular Technology Conference, pp. 1-5, 2013.

[5] M.-H. T. Tsung-Hui Chuang and C.-Y. Chuang, "Group-Based Uplink Scheduling for Machine-Type communications in LTE-Advanced Networks," IEEE Conference of WAINA, pp. 652-657, 2015.

[6] 3GPP TR 22.368 V11.2.0, "Service requirements for Machine-Type Communication," June 2011.

[7] 3GPP TR 37.868 V11.0.0, "Study on RAN Improvements for Machine-Type Communication," September 2011.

[8] F. H. Kan Zheng and W. Wang, "Radio resource allocation in LTE-advanced cellular networks with M2M communications," IEEE Communication Magazine, pp. 184-192, July 2012. 
[9] A. X. J. P. M.Zubair Shafiq, Lusheng Ji and J. Wang, "A First Look at Cellular Machine-tomachine Traffic-Large scale Measurement and characterization," In proceedings of the ACM International Conference on Measurements and Modeling of Computer systems, pp. 17-28, June 2013.

[10] K. j. N. H. Geng Wu, Shilpa Talwar and K. D.Johnson, "M2M: From Mobile to Embedded Internet ," IEEE Communication Magazine, April 2011.

[11] 3GPP TR 23.888 V11.0.0, "System Improvements for Machine-Type Communications," September 2012.

[12] L. Y. K. Andreas Kunz, Hyunsook Kim and S. S.Husain, "Machine Type Communications in 3GPP," GC'12 Workshop, 2012.

[13] Y. H. Ahmed Amorkrane, Adlen Ksentini and T. Taleb, "Congestion Control for Machine Type Communications," 2012 IEEE Internation Conference of Communication (ICC), pp. 778-782, 2012.

[14] B.Pareglio, "Machine-to-machine(m2m) communications," ETSI TC M2M Workshop, October 2011.

[15] 3GPP TS 23.221 V11.3.0, "Architectural Requirements," July 2014.

[16] 3GPP TS 23.682 V11.5.0, "Architecture enhancements to fasilitate communication with packet data networks and applications," September 2013.

[17] ZTE,R2-104662, "MTC Simulation Results with Specific Solutions," August 2010.

[18] CMCC TSG R2-113197, "Performance Comparison of Access Class Barring and MTC Specific Backoff Schemes for MTC," August 2010.

[19] R. S. Anna Larmo, "RAN overload control for Machine Type Communications in LTE," GC'12 Workshop:Second International Workshop on Machine-to-Machine Communications, pp. 1751-1756, 2012. 
[20] L. Z. L. H. Wu Xianfeng, Wang Yinfeng and R. Xianyi, "VMIMO scheme for Michine-toMachine on Cellular Network," International Journal of Computer Science, vol. 10, pp. 2630, May 2013.

[21] J.-H. Huang, Y.-Y. Chen, Y.-T. Huang, P.-Y. Lin, Y.-C. Chen, Y.-F. Lin, and S.-C. Yen, "Rapid Prototyping for Wildlife and Ecological Monitoring," IEEE Systems Journal, vol. 4, pp. 198-209, June 2010.

[22] 3GPP TS 36.300 V11.7.0, "Evolved Universal Terrestrial Radio Access (E-UTRA)and Evolved Universal Terrestrial Radio Access Network (E-UTRAN);Overall description;Stage $2, "$ September 2013.

[23] C.-h. L. Jen-Po Cheng and T.-M. Lin, "Prioritized Random Access with Dynamic Access Barring for RAN Overload in 3GPP LTE-A Networks," IEEE:Internatinal Workshop on Machine-to-Machine Communications, 2011.

[24] C.-Y. K. Shao-Yu Lien, Tzu-Huan Liau and K.-C. Chen, " Enhanced Cooperative Access Class Barring and Traffic Adaptive Radio Resource Management for M2M Communication over LTE-A," APSIPA, October 2013.

[25] L. A. Andres Laya and J. Alonso-Zarate, "Is the random Access Channel of LTE and LTEA Suitable for M2M communications? A Survey of Alternatives," IEEE Communication Surveys and Tutorials, vol. 16, no. 1, 2014.

[26] A.-E. M. T. Mohammad Tauhidul Islam and S. Akl, "A Survey of Access Managemennt Techniques in machine Type Communications," IEEE Communications Magazine, April.

[27] A. A. Fatima Hussain and R. Vannithamby, "Medium access control techniques in M2M communication: survey and critical review," Emerging Telecommunication Tecnologies, September.

[28] 3GPP TR 22.806 V0.3.0, "Study on Application specific congestion control for data communication," August 2013. 
[29] 3GPP TR 22.368 V10.4.0, "Service Requirements for Machine-Type Communication," March 2011.

[30] M.Cheng, H. G.Lin, and C.Hsu, "Performance Evaluation of radio access network overloading from machine type communications in LTE-A Networks," Wireless Communications and Networking Conference Workshops (WCNCW), pp. 284-252, April 2012.

[31] G.-Y. L. Ming-Yuan Cheng and H.-Y. Wei, "Overload control for machine-typecommunications in LTE-advanced system," IEEE Communication Magazine, pp. 38-45, June 2012.

[32] C. S. Nuno K.Pratas, Henning Thomsen and P. Popovski, "Code-Expanded Random Access for Machine-Type Communications," GC'12 Workshop:Second International Workshop on Machine-to-Machine Communications, pp. 1806-1811, 2012.

[33] C. S. Henning Thomsen, Nuno K.Pratas and P. Popovski, "Code-Expanded Radio Access Protocol for Machine-to-Machine Communications," Emerging Telecommunication Tecnologies, vol. 24, pp. 355-365, 2013.

[34] S. Y. Shin and D. Triwicaksono, " Radio resource control scheme for Machine-to-Machine communication in LTE Infrastructure," ICTC,2012, pp. 1-6, October 2012.

[35] M. J. Anthony Lo, Yee Wei Law and M. Kucharzak, "Enhanced LTE-Advanced Random Access Mechanism for Massive Machine-to-Machine (M2M) Communications," 27th World Wireless Research, 2011.

[36] M.-H. F. Umesh Phuyal, Ali T Koc and R. Vannithamby, "Controlling access overload and signalling congestion in m2m networks," Signals, System and Computers (ASILOMAR), 2012, pp. 591-595, 2012.

[37] M. Hasan and E. Hossain, " Random access for Machine-to-Machine communication in LTE-Advanced network:issues and approaches," IEEE Communications Magazine, pp. 8692, June 2013. 
[38] 3GPP TS 36.300, "Evolved Universal Terrestrial Radio Access(E-UTRA) and Evolved Universal Terrestrial Radio Access Network(E-UTRAN);Overall description."

[39] K.-C. C. Shao-Yu Lien and Y.Lin, "Towards Ubiquitous Massive Accesses in 3GPP Machineto-Machine Communication," IEEE Communication Magazine, special issue on recent progress in machine-to-machinine communication, April 2011.

[40] K. W. Yi-Huai Hsu and Y.-C. tseng, "Cooperative Access Class Barring for Machine-toMachine Communication," IEEE Transaction on Wireless Communication, vol. 11, January 2012 .

[41] Y. H.-A. Adlen Ksentini and T. Taleb, "Cellular-Based Machine-to-Machine:Overload Control," IEEE Networks, vol. 26, pp. 54-60.

[42] C.-Y. Tu, C.-Y. Ho, and C.-Y. Huang, "Energy-efficient algorithms and evaluations for massive access msnagement in cellular based Machine-to-Machine communications," Vehicular Technology Conference (VTC Fall), pp. 1-5, 2011.

[43] T. Taleb and A. Kasentini, "An efficient scheme for MTC overload control based on signalling message compression," Global Communications Conference, pp. 342-346, December 2013.

[44] T.Ogawa, H. S.Kotabe, H.Nakamuraand, and T.Shibata, "Fair congestion control method for terminal groups with wireless random access in M2M network," Communications and Information Technologies (ISCIT), pp. 1069-1074, October 2012.

[45] W. W. Bo Yang, Guangxi Zhu and Y. Gao, "M2M access performance in LTE-A system," Transactions on Emerging Telecommunications Technologies, November 2013.

[46] S.-L. T. Ray-Guang Cheng, Chia-Hung Wei and F.-C. Ren, "RACH Collision Probability for Machine-type Communications," IEEE-Vehicular Technology Conference, pp. 1-5, 2012.

[47] W. W. Kan Zheng, Fanglong Hu, W. Xiang, and M. Dohler, "Radio Resource allocation in LTE-Advanced Cellular networks with M2M Communications," IEEE Communicatiom Magazine, vol. 50, no. 7, pp. 184-192, 2012. 
[48] C.-Y. Oh and D. Hwang, "Joint Access Control and Resource Allocation for Concurrent and Masive Access of M2M Devices," IEEE Transaction on Wireless Communications, no. 99, 2015.

[49] A. S. Lioumpas and A. Alexiou, "Uplink Scheduling for Machine-to-Machine Communicatuions in LTE-based Cellular Systems," IEEE-International Workshop on Machine-toMachine Communications, pp. 353-357, 2011.

[50] L. Ferdouse and A. Anpalagan, "Relay Selection Based on Bayesian Decision Theory in Cooperative Wireless Networks," IEEE-Canadian Journal of Electrical and Computer Engineering, vol. 38, no. 2, pp. 116-124, 2015. 